\title{
SAPHIRE 8 New Features and Capabilities
}

Curtis Smith James Knudsen Dan O'Neal Tracen Peterson Kurt Vedros

August 2008

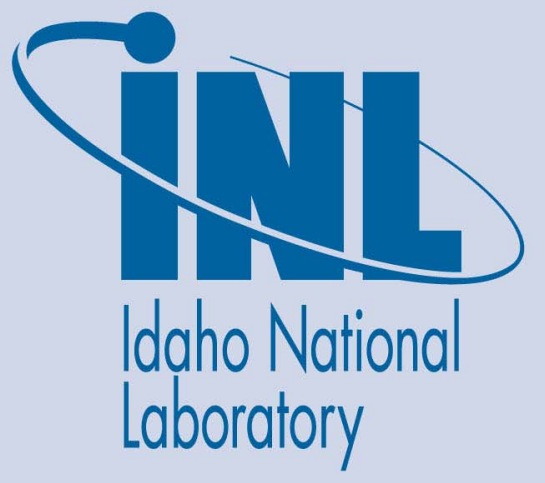

The INL is a U.S. Department of Energy National Laboratory operated by Battelle Energy Alliance 


\title{
SAPHIRE 8 New Features and Capabilities
}

\author{
Curtis Smith \\ James Knudsen \\ Dan O'Neal \\ Tracen Peterson \\ Kurt Vedros
}

August 2008

\section{Idaho National Laboratory \\ Idaho Falls, Idaho 83415}

http://www.inl.gov

\author{
Prepared for the \\ Division of Risk Analysis \\ Office of Nuclear Regulatory Research \\ U.S. Nuclear Regulatory Commission \\ Washington D.C. 20555 \\ Job Code N6423
}




\section{DISCLAIMER NOTICE}

This report was prepared as an account of work sponsored by an agency of the United States Government. Neither the United States Government nor any agency thereof, or any of their employees, makes any warranty, expressed or implied, or assumes any legal liability of responsibility for any third party's use, or the results of such use, or any information, apparatus, product or process disclosed in this report, or represents that its use by such third party would not infringe privately owned rights. 


\section{Table of Contents}

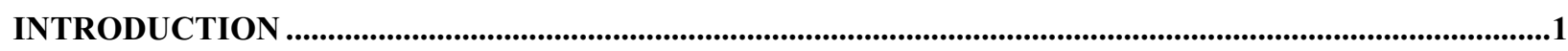

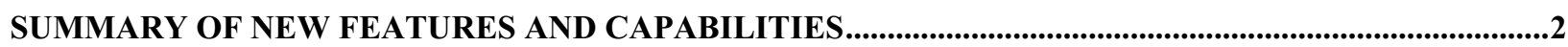

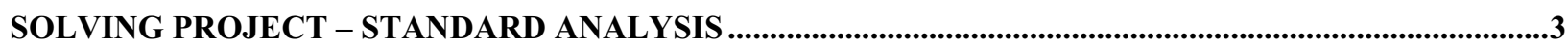

MODEL EDITING

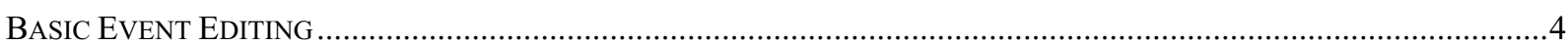

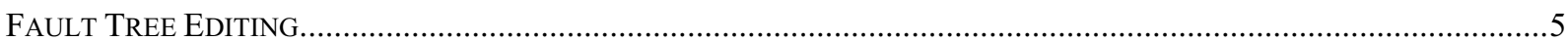

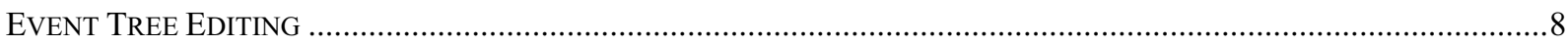

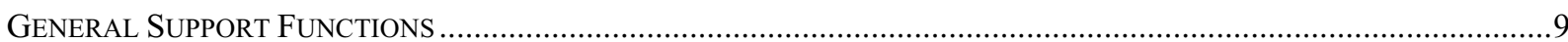

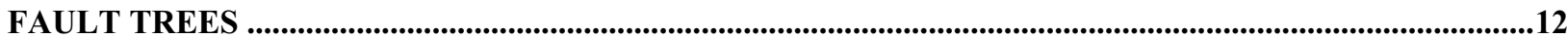

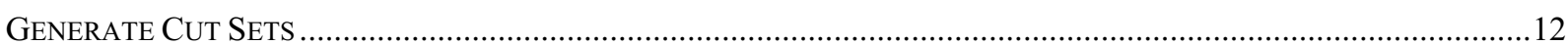

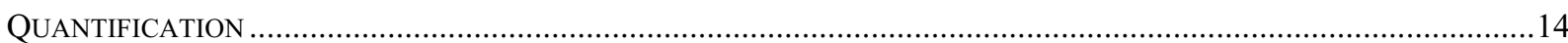

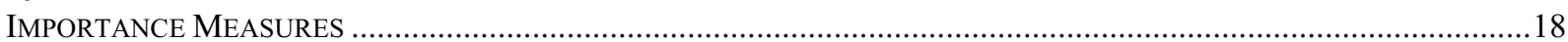

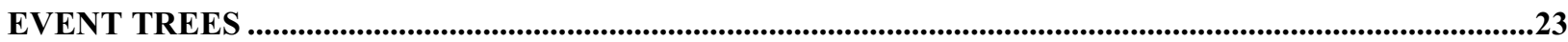

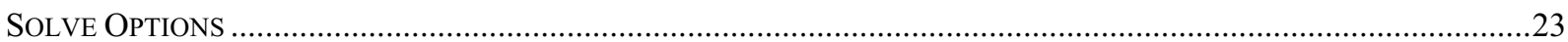

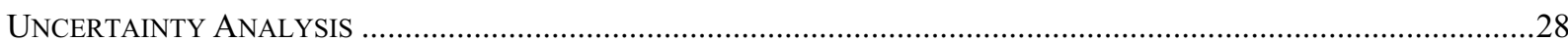

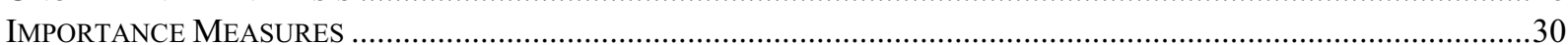

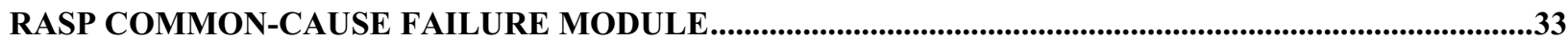

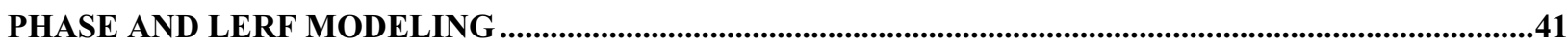

EXTERNAL EVENTS MODELING VIA AN ACCIDENT SEQUENCE MATRIX .......................................42

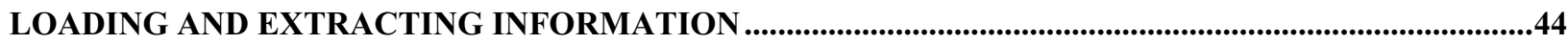

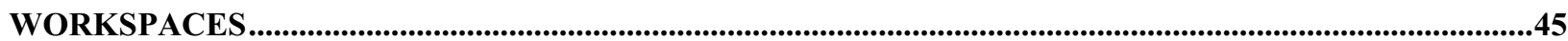

SOLVING PROJECT - SIGNIFICANCE DETERMINATION PROCESS (SDP).............................................46

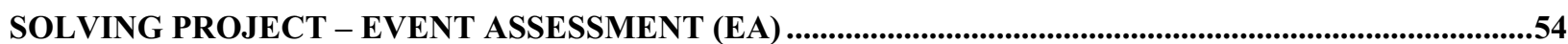

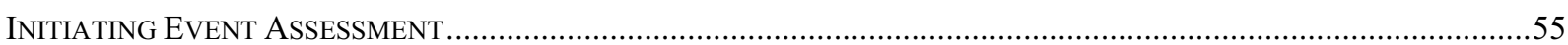

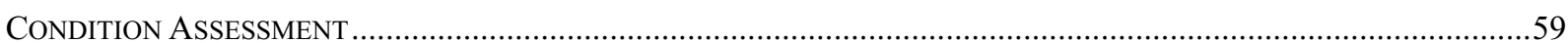

SOLVING PROJECT - GENERAL ANALYSIS …........................................................................................63 


\section{$\underline{\text { Table of Figures }}$}

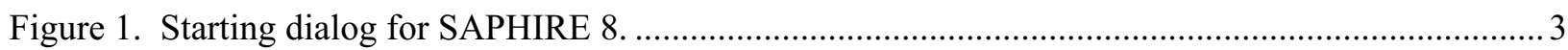

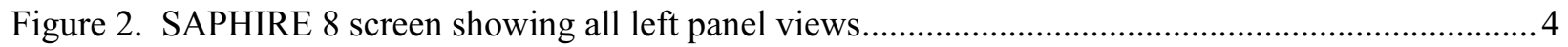

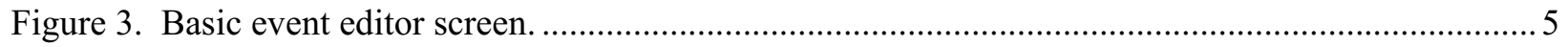

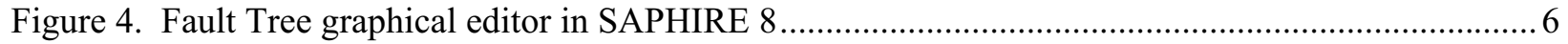

Figure 5. Basic Event editing form in the Fault Tree graphical environment............................................. 7

Figure 6. AND Gate cursor in Fault Tree editing screen .................................................................. 8

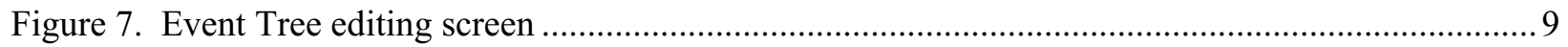

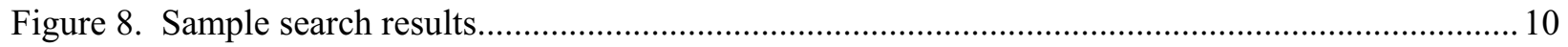

Figure 9. Bookmark example

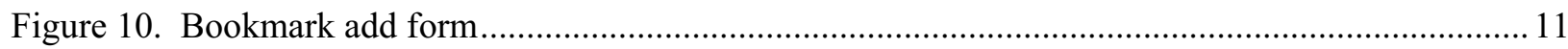

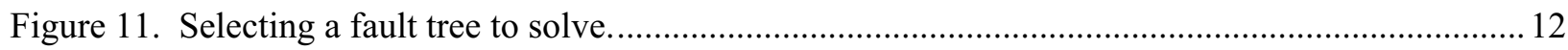

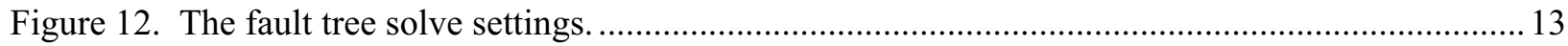

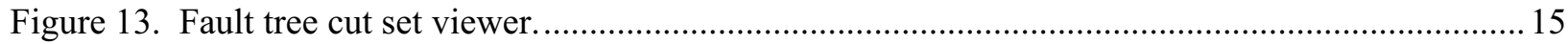

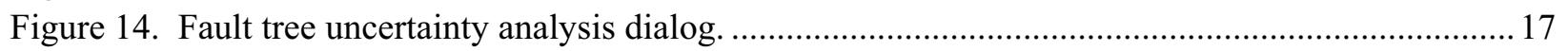

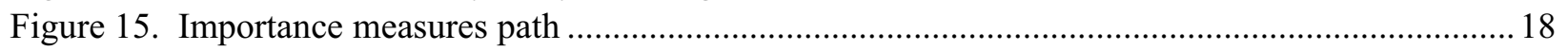

Figure 16. Behavior of FV and RRR as basic event probabilities are varied. ...................................... 19

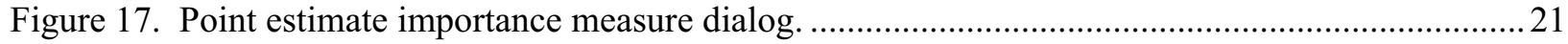

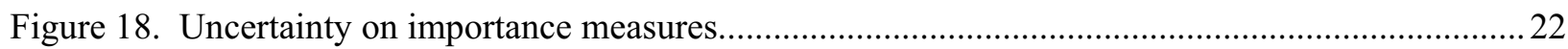

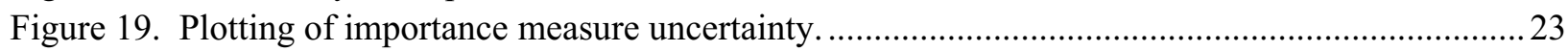

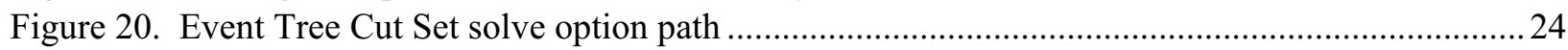

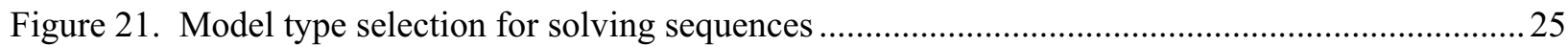

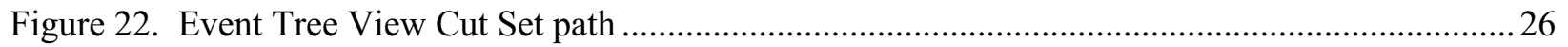

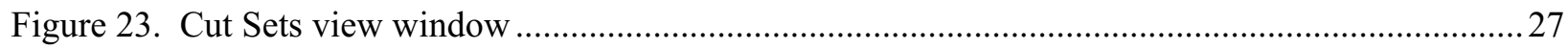

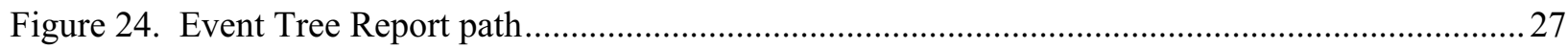

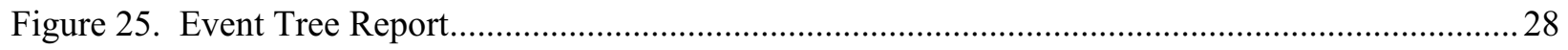

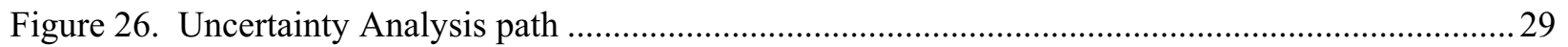

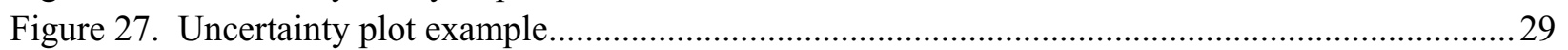

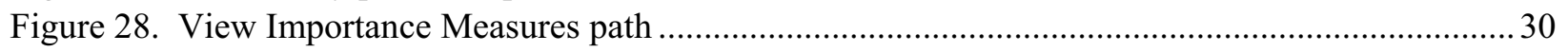

Figure 29. Importance Measures based on point estimate .............................................................. 31

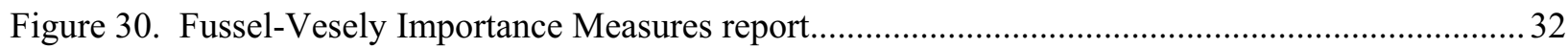

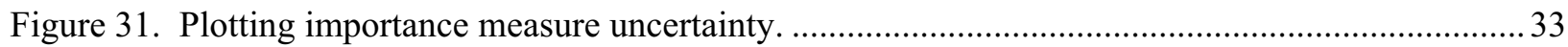

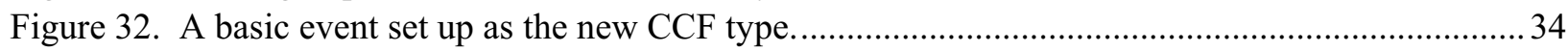

Figure 33. Fault tree with CCF representing the example system. .................................................... 35

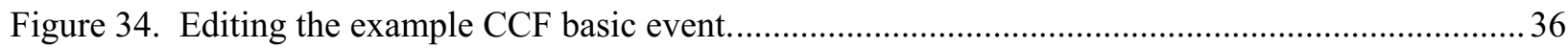

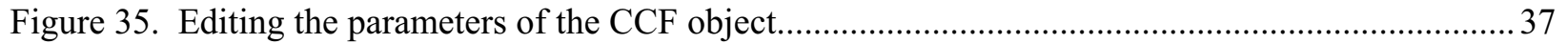

Figure 36. Fault Tree with MOV CCs independent and MY_CCF included .......................................... 40

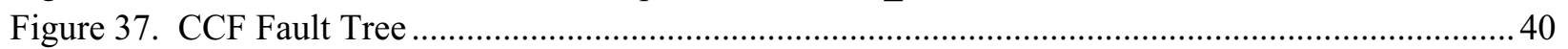

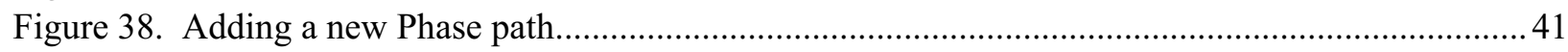

Figure 39. Example Event Tree with multiple level End-States ..........................................................42

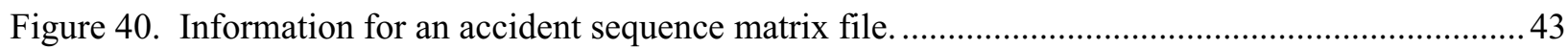

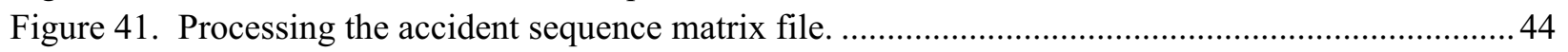

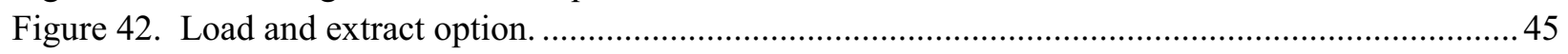

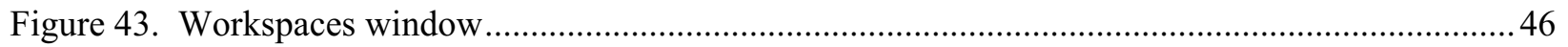

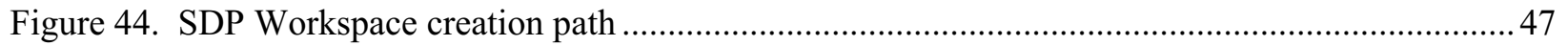




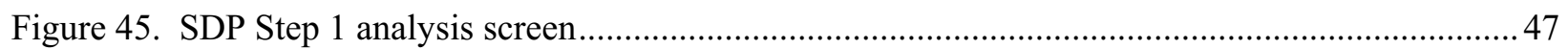

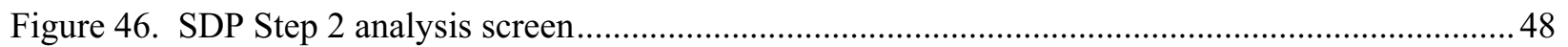

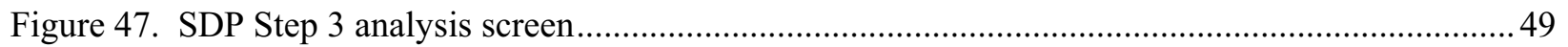

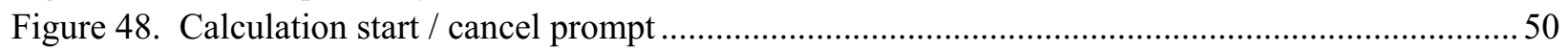

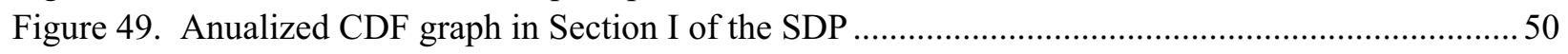

Figure 50. Relative risk change for the condition (Figure III-A of SDP report)..................................52

Figure 51. Percent contribution of initiating events to the increase in CDF (Figure III-B of SDP report) 53

Figure 52. Relative risk change grouped by initiating event (Figure III-C of SDP report) .....................53

Figure 53. Change in annualized CDF as a function of duration (Figure III-D of SDP report) ...............54

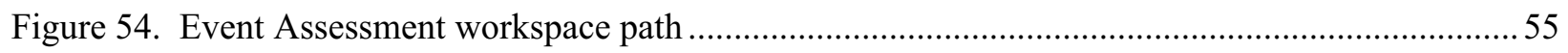

Figure 55. Events Assessment Type analysis screen ....................................................................5

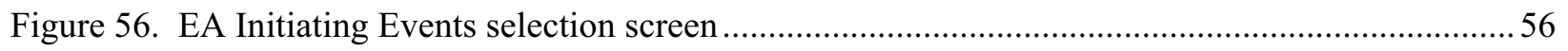

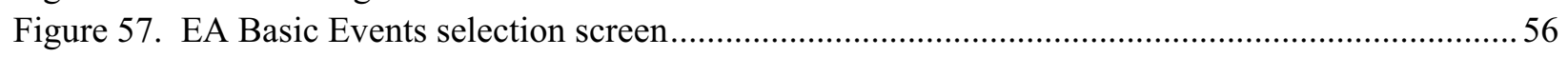

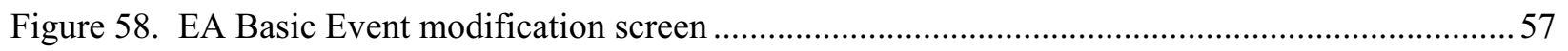

Figure 59. EA Solve options screen for Initiating Event assessment......................................................58

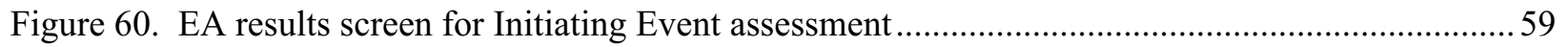

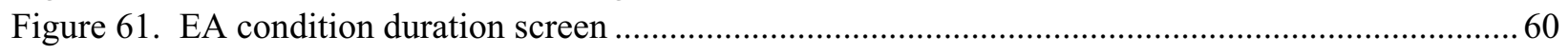

Figure 62. EA Solve options screen for Condition Assessment ........................................................61 61

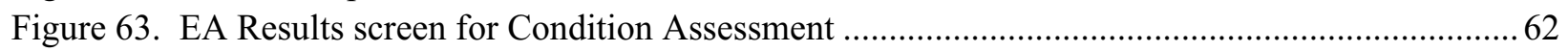

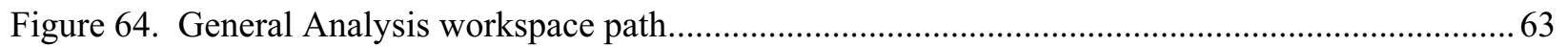

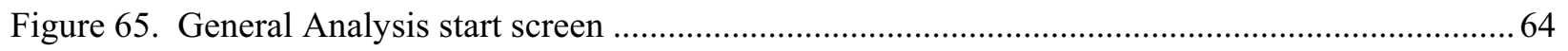

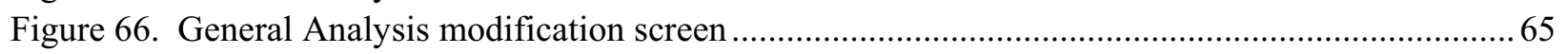

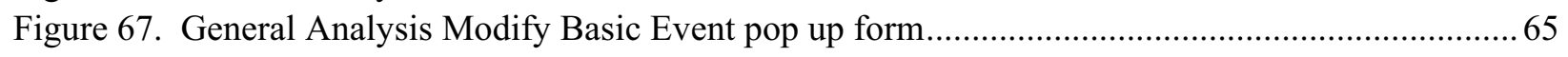

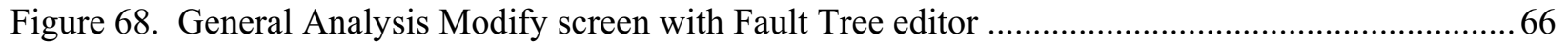

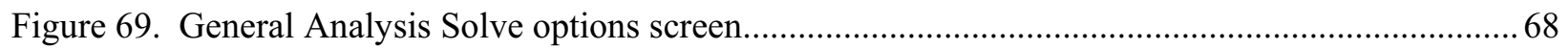

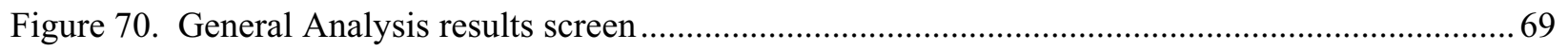

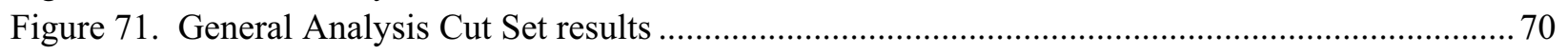

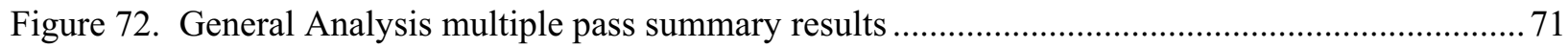

Figure 73. General Analysis multiple pass Cut Set results .................................................................... 72

Figure 74. General Analysis summary results with component to True and multiple pass performed .....73

Figure 75. General Analysis Cut Set results with component to True and multiple pass performed ........74 


\section{INTRODUCTION}

The Systems Analysis Programs for Hands-on Integrated Reliability Evaluations (SAPHIRE) software performs probabilistic risk assessment (PRA) calculations. SAPHIRE is used in support of NRC's riskinformed programs such as the Accident Sequence Precursor (ASP) program, Management Directive 8.3, "NRC Incident Investigation Program," or the Significance Determination Process (SDP). It is also used to develop and run the Standardized Plant Analysis Risk (SPAR) models. SAPHIRE Version 8 is a new version of the software with an improved interface and capabilities to support risk-informed programs.

SAPHIRE Version 8 is designed to easily handle larger and more complex models. Applications of previous SAPHIRE versions indicated the need to build and solve models with a large number of sequences. Risk assessments that include endstate evaluations for core damage frequency and large, early release frequency evaluations have greatly increased the number of sequences required. In addition, the complexity of the models has increased since risk assessments evaluate both potential internal and external events, as well as different plant operational states. Special features of SAPHIRE 8 help create and run integrated models which may be composed of different model types.

SAPHIRE 8 includes features and capabilities that are new or improved over the current Version 7 to address the new requirements for risk-informed programs and SPAR models. These include:

- Improved User Interfaces

- Model development

- Methods

- General Support Features

The user interfaces are re-designed to utilize the main screen with flexible use of window sizing. Models loaded into SAPHIRE 8 are displayed on the main screen. The risk analyst can view and edit the model logic and perform standard analyses (e.g., sequence solving, uncertainty analysis, etc.) all from the main screen with use of pop-up forms and the available windows.

On the main screen, different user interfaces can be selected for performing analysis on the loaded model database. An interface was developed for: (1) Significance Determination Process (SDP) phase 2 analyses with the SPAR models; (2) event assessments; and, (3) other types of analyses. The user interfaces are designed to be used at different levels of complexity. The SDP interface is simplified when compared to the current Graphical Evaluation Module (GEM) in SAPHIRE 7. The Events Assessment (EA) interface retains all the capabilities of the GEM interface. It supports streamlined analyses for the ASP program, as well as other risk-informed programs. A third interface, General Analysis, provides an additional level of capability by allowing changes to the model's underlying logic for a risk assessment. The reports for each interface have been tailored for their intended applications.

Features and capabilities have also been improved for SPAR model development and use. External events models can be built more expeditiously. External event modeling involves repetitive uses of some modeling features, and a capability was developed to make this easier for the model developer. In addition, the capability was added to build large, early release frequency (LERF) models beyond the 
bridge event tree/containment event tree approach. Also, as previously mentioned, SAPHIRE Version 8 has been designed to support integrated models which combine different model types (e.g., internal and external events models). These integrated models can be constructed all within SAPHIRE, or, alternatively, different parts of the model can be built on different computers and then pieced together. Any combination of model types can be solved, and powerful graphical editors allow examination of the underlying logic.

New modeling and calculation methods have also been implemented. The capability to perform phase mission time analysis has been incorporated in support of Level 1 and LERF SPAR models. This capability will also be useful for low power and shutdown models. The common cause failure methodology for the Risk Assessment Standardization Project (RASP) has also been incorporated. Common cause failure probabilities will be automatically adjusted for sequence flag sets. In addition, the sequence solving routine has been improved.

Finally, the functionality, in general, that was available in Version 7 is available in Version 8, but in an improved manner. One of the strengths of SAPHIRE lies in its computation capabilities. Version 8 not only taps into these capabilities, but also is better positioned to leverage these capabilities for non-expert users.

\section{SUMMARY OF NEW FEATURES AND CAPABILITIES}

Features new to SAPHIRE 8 include:

- flexible window interface with multiple open dialogs and resizable windows

- the capability of model manipulation from the main screen

- the concept of workspace files which save analyses and can be built upon without affecting the base model

- more powerful and easy to use graphical editor

Capabilities new to SAPHIRE 8 include:

- workspaces that are independent of the basic model, each other, and can compare cases within their own environment

- powerful search function

- multiple objects (trees, searches, etc...) open and accessible at the same time

- simplified SDP interface for SPAR models from main screen

- streamlined ASP analysis

- reports tailored to the applications with increased graphical presentation

- improved graphical representation of uncertainty

- RASP Common Cause Failure modification module

- improved ability to piece models together from parts created on multiple computers

- improved external event modeling 


\section{SOLVING PROJECT - STANDARD ANALYSIS}

Solving a SAPHIRE 8 project can be performed directly in the project or by setting up a new general analysis in a user selectable workspace. The differences between these two analysis options will be discussed below.

The starting point in analyzing an existing model is to load the project into SAPHIRE 8 as shown in the entry menu (Figure 1). The project specified in the Current Project field is opened or a new project can be selected by selecting the Browse option and scrolling through the folders until the project is located.

The next step to opening the project is to determine what type of analysis is going to be performed. To perform a standard analysis (similar to SAPHIRE 7) an analysis option is specified in the drop down box and then the green arrow below this option is clicked. Otherwise, the "Modify a Reliability or Risk Analysis Project" option is selected. The "modify" option allows for project creation and manipulation along with all types of analyses. Discussions of the analyses available are found in the sections on Solving Projects - Significance Determination Project, Event Assessment, and General Analysis.

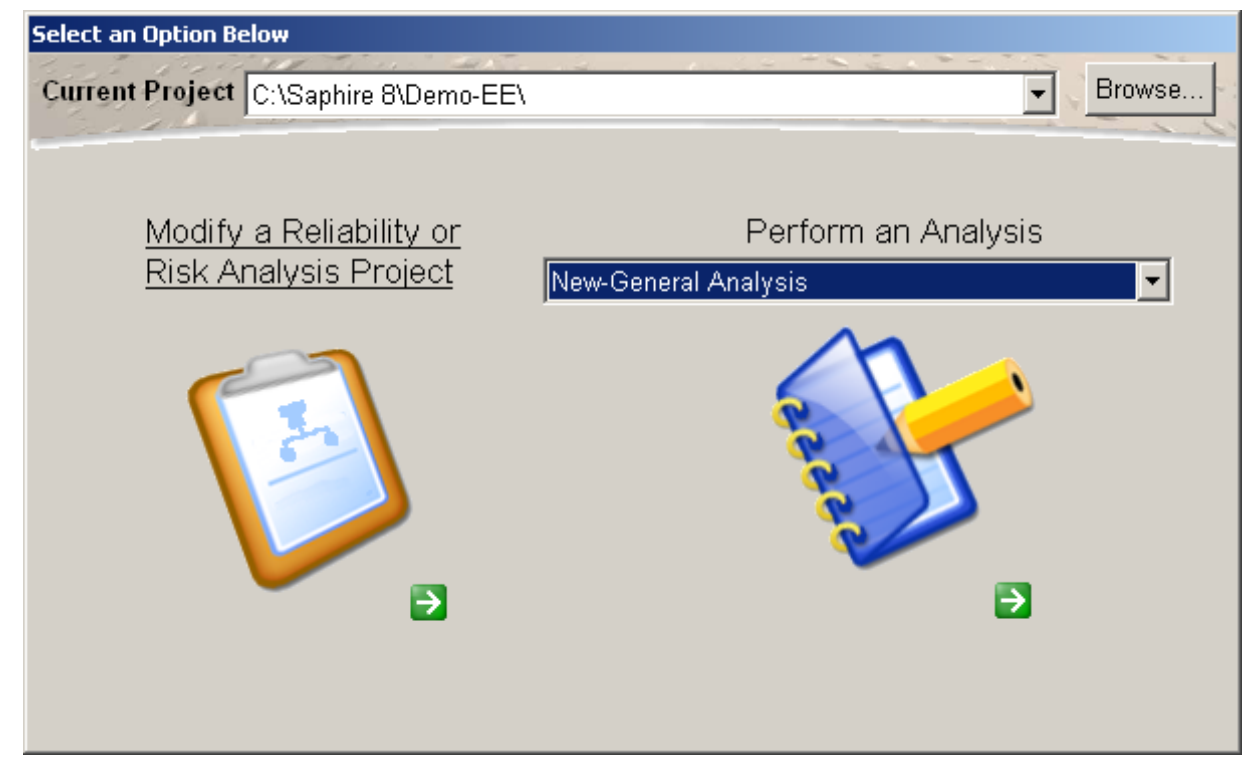

Figure 1. Starting dialog for SAPHIRE 8.

\section{MODEL EDITING}

SAPHIRE 8 has provided an improved interface to modify a reliability or risk analysis project's database. The windows in the left panel of the main screen are expandable and collapsible. The whole left panel can be resized as well by clicking and dragging the edge. Figure 2 shows the model windows that can be used in the left panel for model manipulation and for creating and running analyses. 


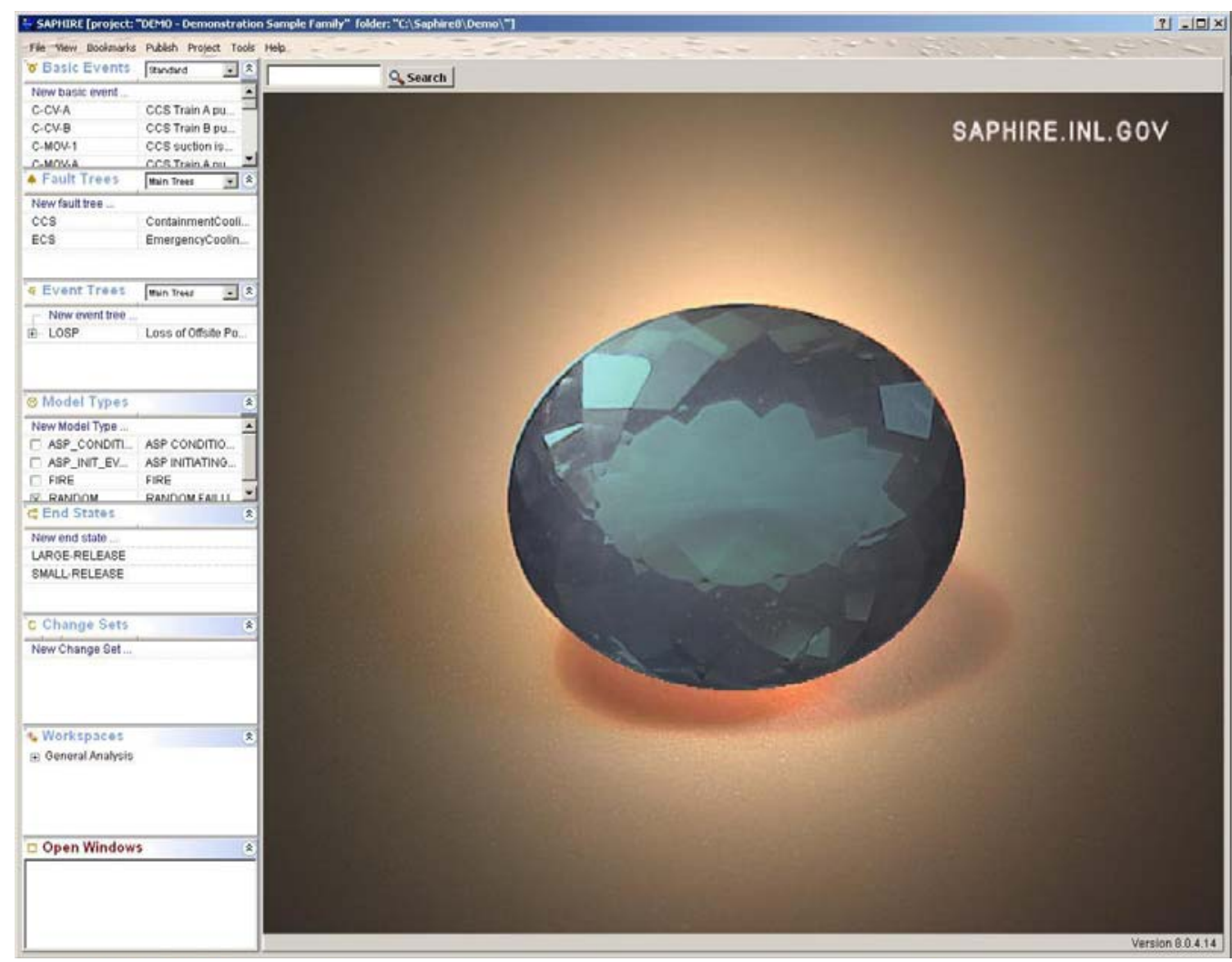

Figure 2. SAPHIRE 8 screen showing all left panel views

\section{Basic Event Editing}

The basic event editor is segregated into five tabs (see Figure 3) for the different types of basic event information.

$\underline{\text { Failure Model }}$

$\underline{\text { Attributes }}$

Applicability

$\underline{\text { Notes }}$

Summary 


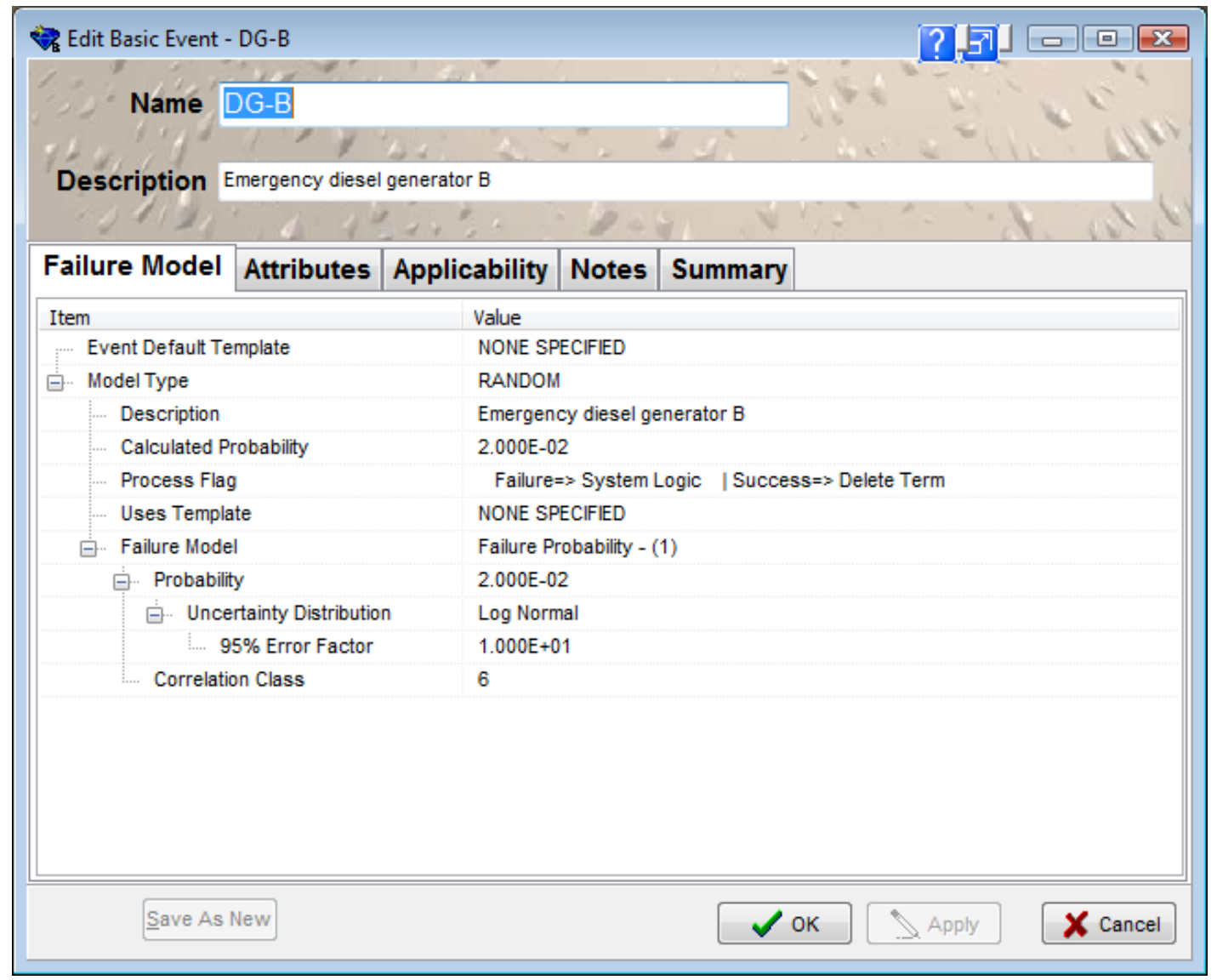

Figure 3. Basic event editor screen.

The "failure model" option allows the user to edit the failure model(s) for basic events. The Failure Model page consists of a tree-view control listing all of the applicable options required to specify the event's failure model. In SAPHIRE 8, a failure model is assigned to each event's Model Type (if more than one, as assigned in the Applicability tab). Each failure model can have a description. The Calculated Probability is not editable, it will be determined from the settings below it.

The Failure Model controls how the event's probability is calculated. A new failure model in SAPHIRE 8 is the "R" type, or RASP CCF. This model is described later in this report.

\section{Fault Tree Editing}

The capability to modify the model and the functionality of the graphical editor has improved. The list of Fault Trees is shown in the fault tree window in the left panel. Open a Fault Tree by double clicking on the name. As shown in Figure 4 for the Demo CCS Fault Tree, the basic events can be stacked. Transfers can also be stacked. The transfer icon is double clicked to follow the transfer by opening up the transfer tree graphics. SAPHIRE 8 keeps all of the trees open in the right panel. Multiple objects are kept accessible as all open trees and other windows in the right panel are listed in the Open Windows window at the bottom of the left panel. Basic Events can be modified through choosing them in the Basic Events 
window or within the graphical editor as shown in Figure 5. The same Basic Event form pops up as would if the BIE was chosen in the Basic Event window.

Fault Trees are also collapsible by clicking on the "-" icon underneath a gate. A "+" icon indicates there are unexpanded gates and/or events, which then are clicked to expand the model.

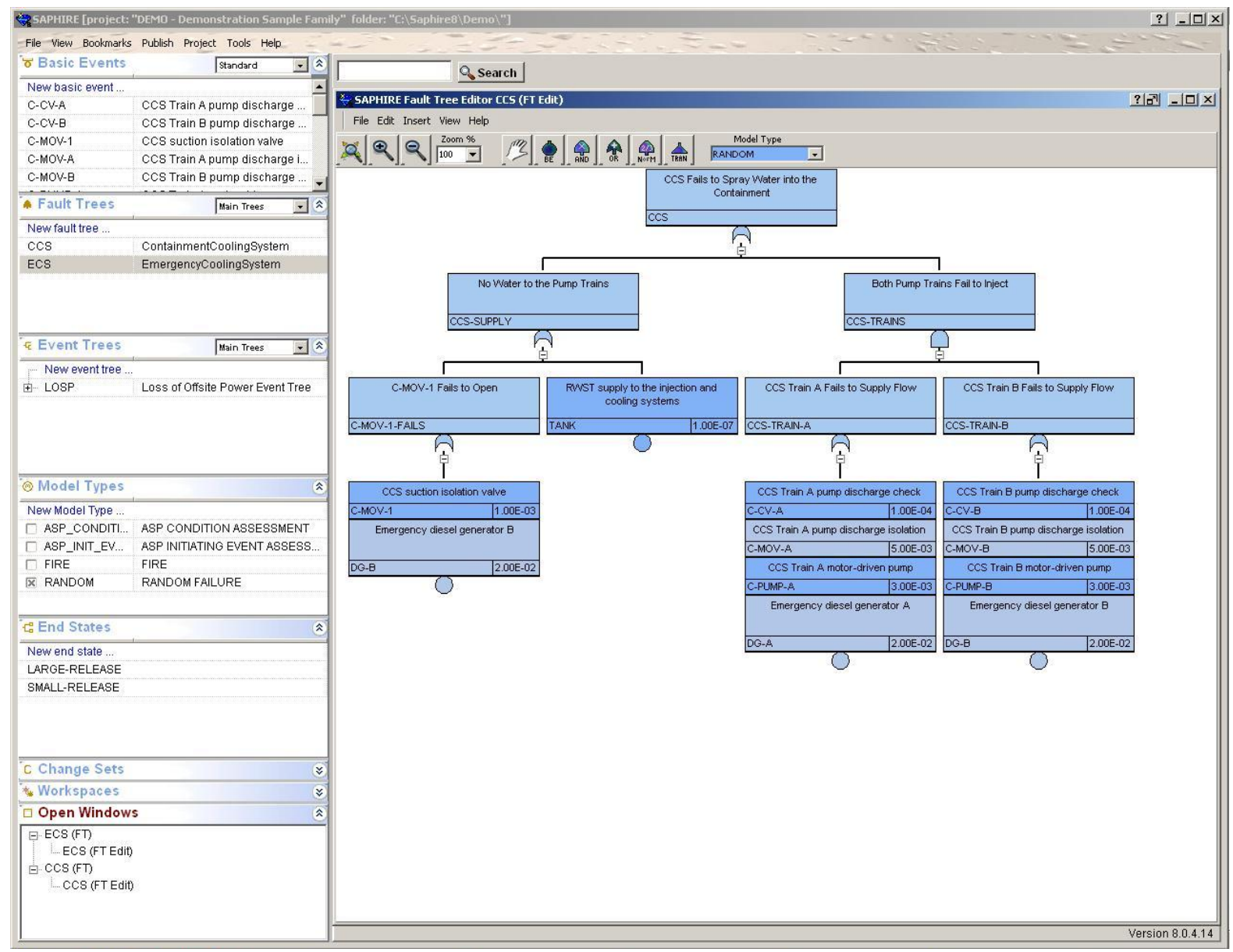

Figure 4. Fault Tree graphical editor in SAPHIRE 8. 


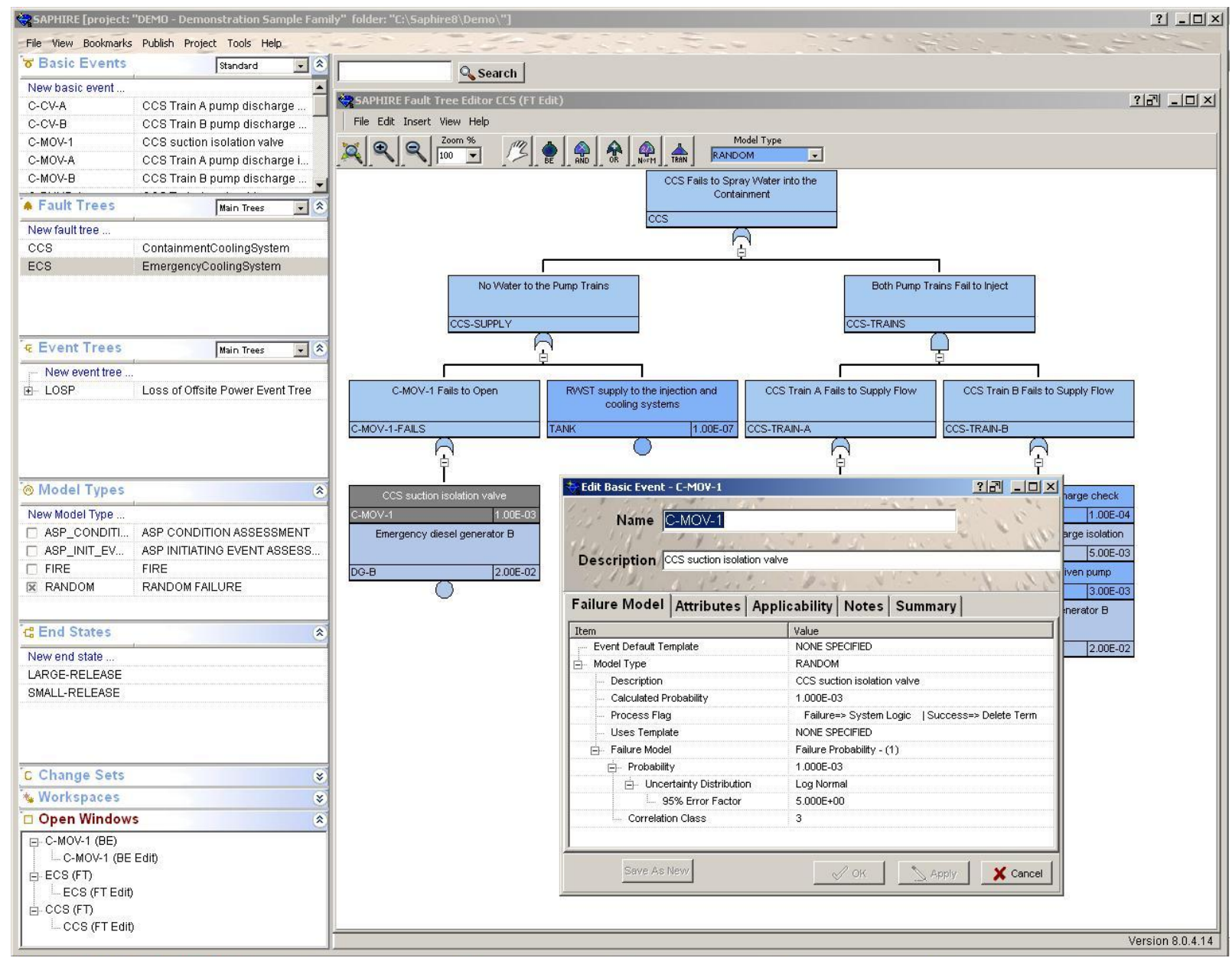

Figure 5. Basic Event editing form in the Fault Tree graphical environment

Adding a gate to a Fault Tree is accomplished by clicking on the button with the desired gate icon at the top of the editing screen. The cursor will show an arrow with a null symbol until it is placed over a gate that can accept the insertion. The cursor will then show a gate symbol and a left click of the mouse inserts the gate (Figure 6). 


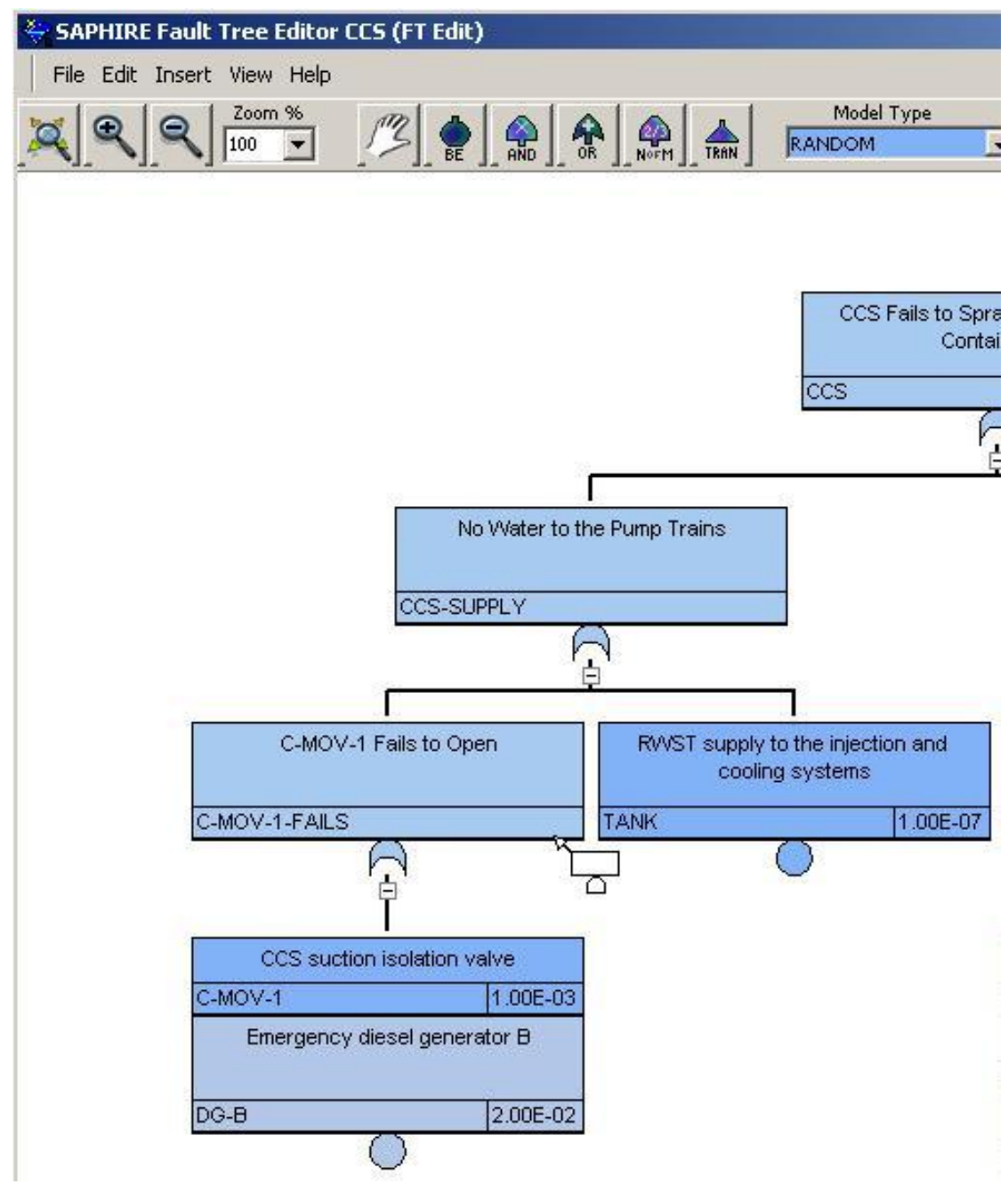

Figure 6. AND Gate cursor in Fault Tree editing screen

\section{Event Tree Editing}

Event Tree editing is accomplished in much the same manner as Fault Tree editing. All of the increased functionality of multiple object accessibility is available as described in the Fault Tree Editing section. Event Trees and Fault Trees can be open at the same time. The branch and top event insertions are made by clicking on the icon for the chosen insertion and placing the resulting cursor (branch up, branch down, top event insert left, top event insert right) over the branch point or top event as applicable, then click to insert. Information and editing of a top event can be accessed by double clicking on the top event box as in SAPHIRE 7. When end-states are double clicked, the frequency and sequence name is also viewable. 


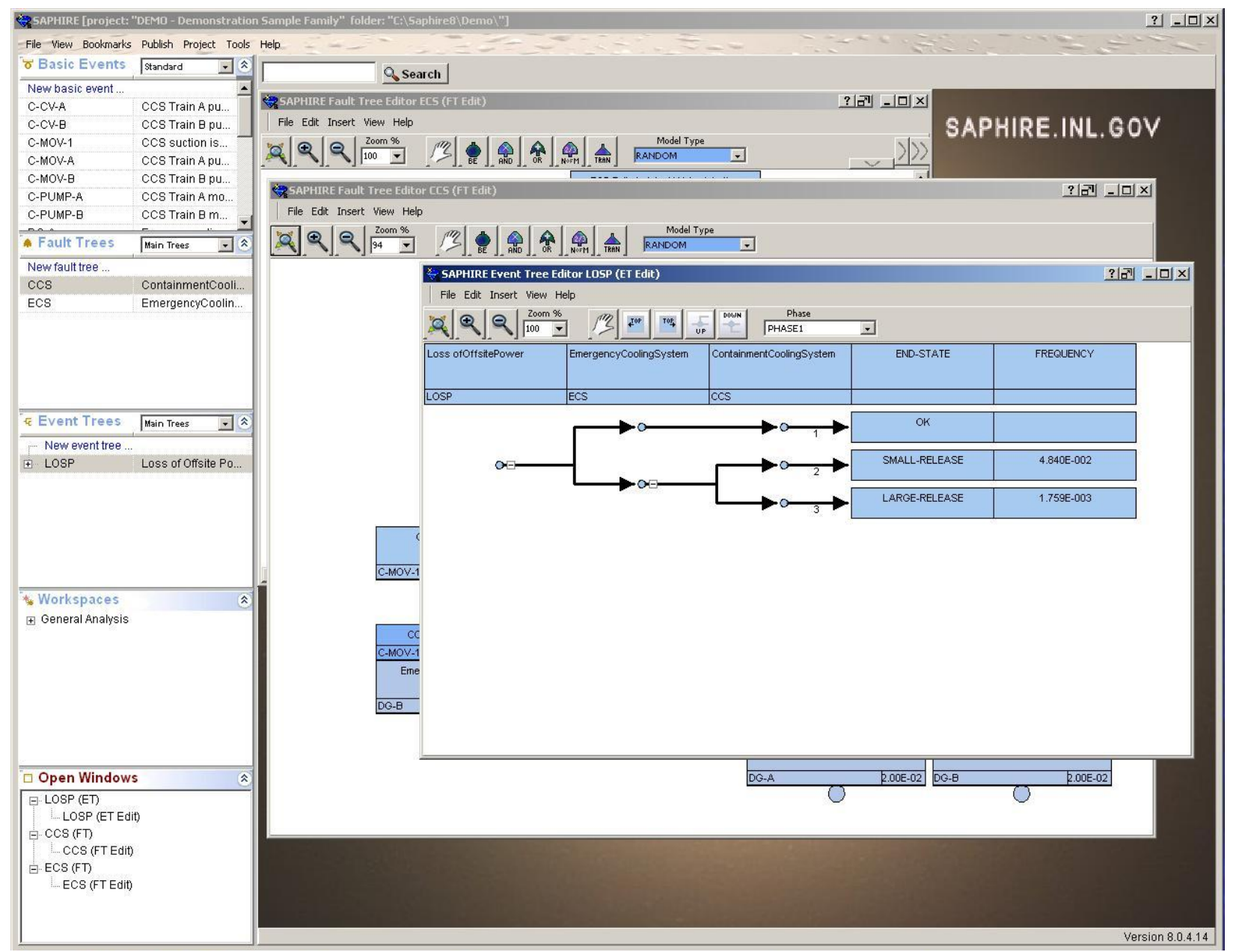

Figure 7. Event Tree editing screen

\section{General Support Functions}

SAPHIRE 8 has a search function that will return any object in the project that matches the search such as tree names, gates and basic events names, and including the matched text in the descriptions. It is not case sensitive. The results can be examined from the search for cross-references in the project, frequencies, quantification methods, and other information including a graphical view if viewing a tree with a movable magnified area. As with any window opened, the search remains open and accessible in the main panel (Figure 8). 


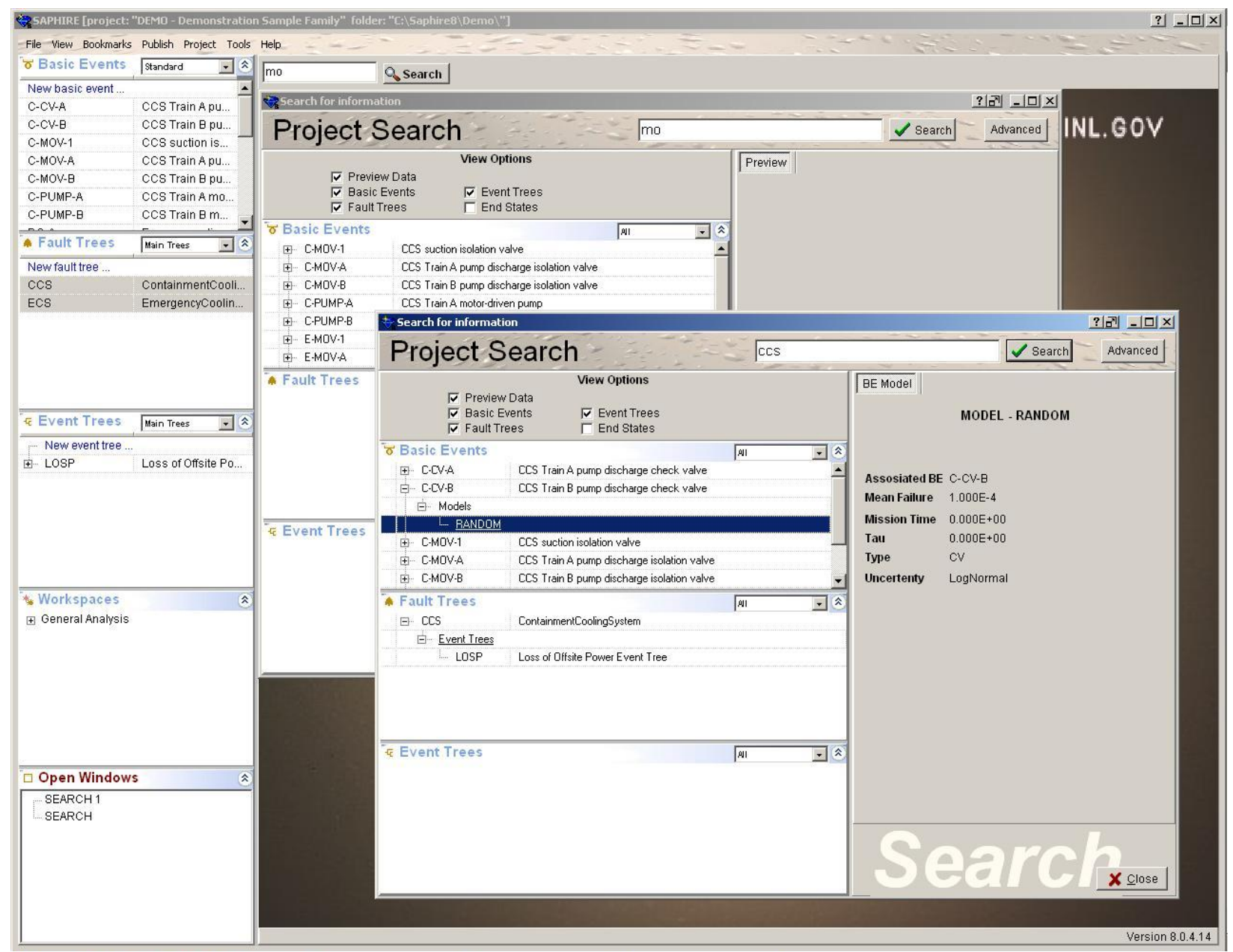

Figure 8. Sample search results

SAPHIRE 8 also has a bookmark option that provides a way to save a group of objects for later retrieval. To have all of the CCS basic events at the click of a bookmark, the CCS are highlighted in the Basic Events window and a right click will bring up the option to bookmark the selection (Figure 9). Name and save the bookmark (Figure 10) and it will be available for retrieval under Bookmarks. When the bookmark is retrieved, the previously highlighted basic events are selected. 


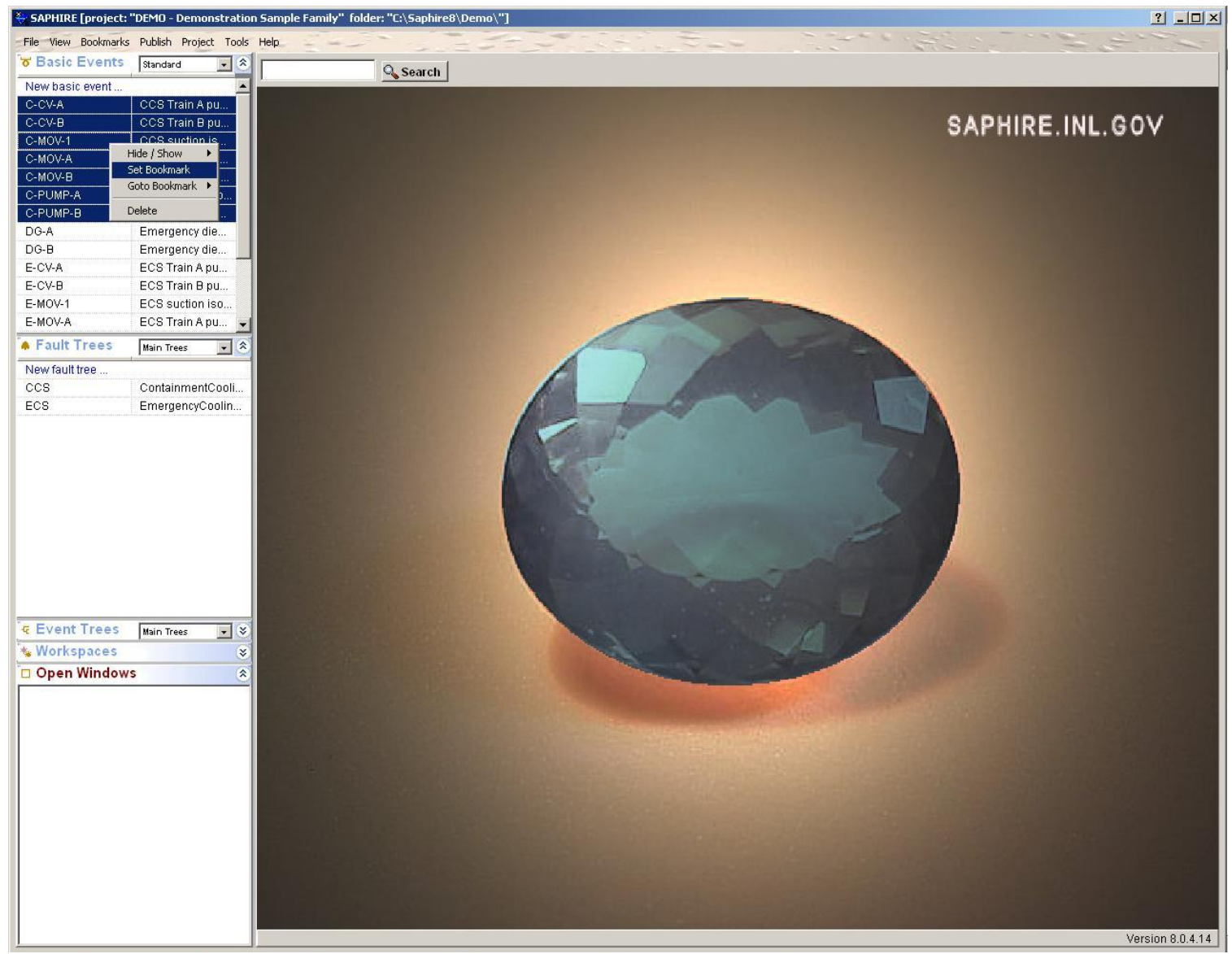

Figure 9. Bookmark example.

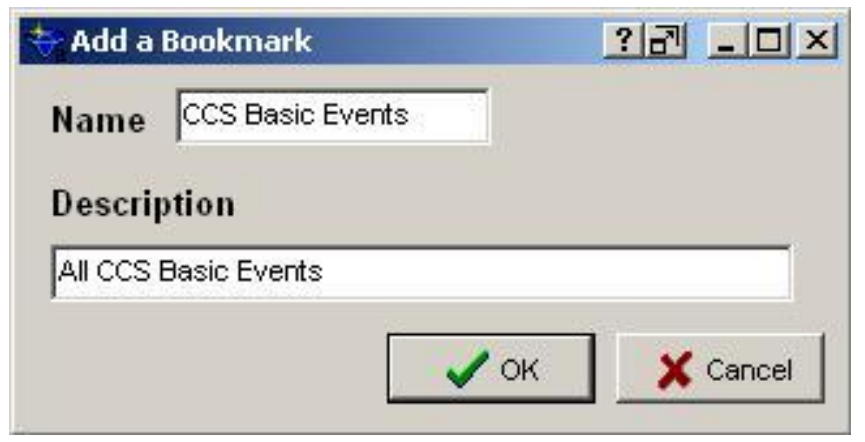

Figure 10. Bookmark add form. 


\section{FAULT TREES}

\section{Generate Cut Sets}

To solve fault tree cut sets from an existing model, the fault tree(s) need to be highlighted and then right click the mouse. A pop-up menu will appear (Figure 11) and then select Solve.

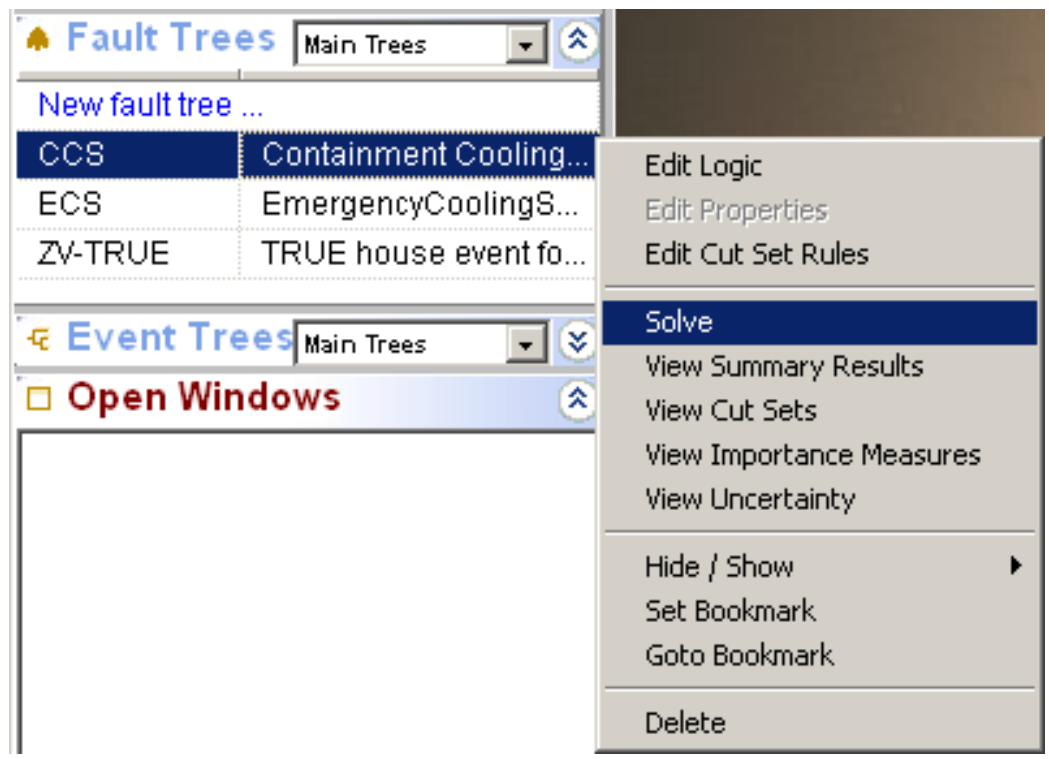

Figure 11. Selecting a fault tree to solve.

The Solve option is different compared to SAPHIRE 7 primarily in that SAPHIRE 8 has all of the solve options located on this one option screen. Within this screen (Figure 12) the following applications are performed: cut set generation, apply recovery rules, cut set update, and base case update. Also, the different model types can be analyzed together or separately.

Note that in SAPHIRE 8, the different model types (e.g., random, seismic, fire, flood) can be solved at the same time (as opposed to SAPHIRE 7). Whenever more than one model type is solved, the cut sets for that particular group of model types is stored uniquely from other groups. For example, if the random and flood model types are selected and solved, cut sets applicable to those two model types will be solved and stored internally in SAPHIRE 8 as a random+flood group. If random and fire model types are selected and solved, these new cut sets will be stored in a random+fire group, leaving the random+flood cut sets untouched for later retrieval. 


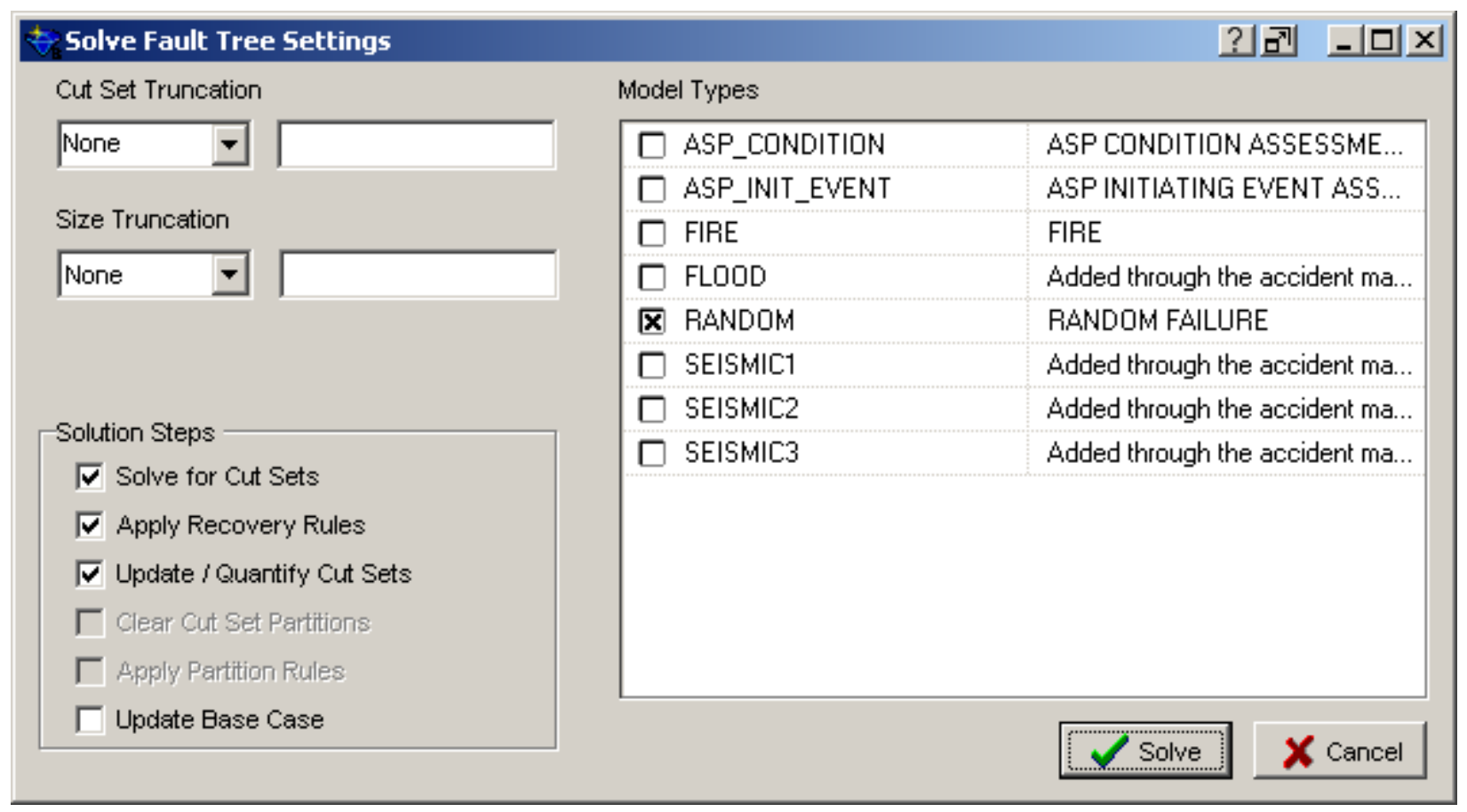

Figure 12. The fault tree solve settings.

\section{Solve Options:}

\section{Cut Set Generation:}

SAPHIRE solves the selected fault tree(s) based on its logic to obtain the minimal cut sets. These resultant cut sets are then quantified or truncated based on the truncation options.

\section{Cut Set Truncation:}

The truncation options are the same as those from SAPHIRE 7. The cut sets can be solved at a Global truncation or a fault tree specific truncation. The Global truncation will solve all fault trees at the specified truncation. The System truncation will solve the fault trees at the fault tree specific truncation. This option requires that a specific truncation be specified for all fault trees. The last truncation option is NONE, which means no truncation will be used in the cut set generation.

The next set of truncation options deal with the number of basic events per cut set. This truncation option is Size (direct number of basic events from logic model) and Zone (based on basic events attributes being specified to zone [i.e., for fire, flood analysis]).

\section{Solution Steps:}

This set of check boxes lets SAPHIRE 8 know what type of solution is requested. Whichever, box(es) is checked, that particular operation will be performed. Multiple boxes can be selected or just one box depending upon the final result. Each box performs the following process:

Solve for Cut Sets - Solve the fault tree for minimal cut sets based on the fault tree logic model. 
Apply Recovery Rules - This check box applies all applicable recovery rules to the fault tree cut sets (Fault Tree specific rule first then Project rule).

Update/Quantify Cut Sets - This option re-evaluates the existing cuts to remove any potential non-minimal cut sets and then quantifies these cut sets using the default calculation.

Update Base Case - This option stores the resultant cut sets into the base case.

\section{Model Types:}

The last option within this screen is what Model Types are to be solved. Each basic event/fault tree can be susceptible to one or more of the Model Types available within SAPHIRE. The default is Random, but multiple Model Types can be selected and SAPHIRE will solve the fault tree based on the Model Type(s) selected. The results will all be grouped together based on the Model Type(s) selected.

\section{Quantification}

The same quantification options are available in SAPHIRE 8 as in SAPHIRE 7. These quantification options are MinCut, Rare Event, and Min/Max. One way to change these options is by right clicking the mouse on the selected fault tree (only one may be selected) and selecting 'Edit Logic'. Inside the Fault Tree Editor, select 'Edit' from the menu bar, and then 'Properties'. The default quantification method may be changed in the lower left corner of the model information editor.

\begin{tabular}{l}
\hline Edit Logic \\
\hline Edit Properties \\
Edit Cut Set Rules \\
\hline Solve \\
View Summary Results \\
View Cut Sets \\
View Importance Measures \\
View Uncertainty \\
\hline Hide / Show \\
Set Bookmark \\
Goto Bookmark \\
\hline Delete
\end{tabular}

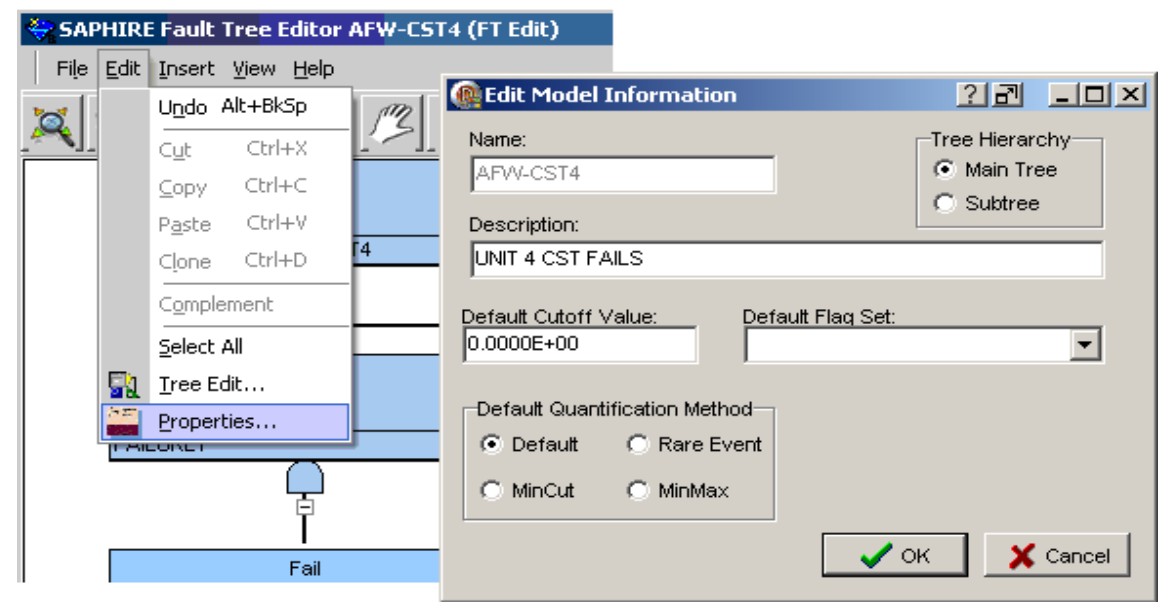

\section{Report:}

There are multiple Report options, View Summary Results and View Cut Sets. 


\begin{tabular}{l} 
Edit Logic \\
Edit Properties \\
Edit Cut Set Rules \\
\hline Solve \\
View Summary Results \\
View Cut Sets \\
View Importance Measures \\
View Uncertainty \\
\hline Hide / Show \\
Set Bookmark \\
Goto Bookmark \\
\hline Delete
\end{tabular}

Once the fault tree(s) have been solved then the cut sets can be viewed. From Figure 13 (View Cut Sets), the fault tree cut sets can be reported by selecting the Report button. This option will display the resultant cut sets for preview and then to the selected printer.

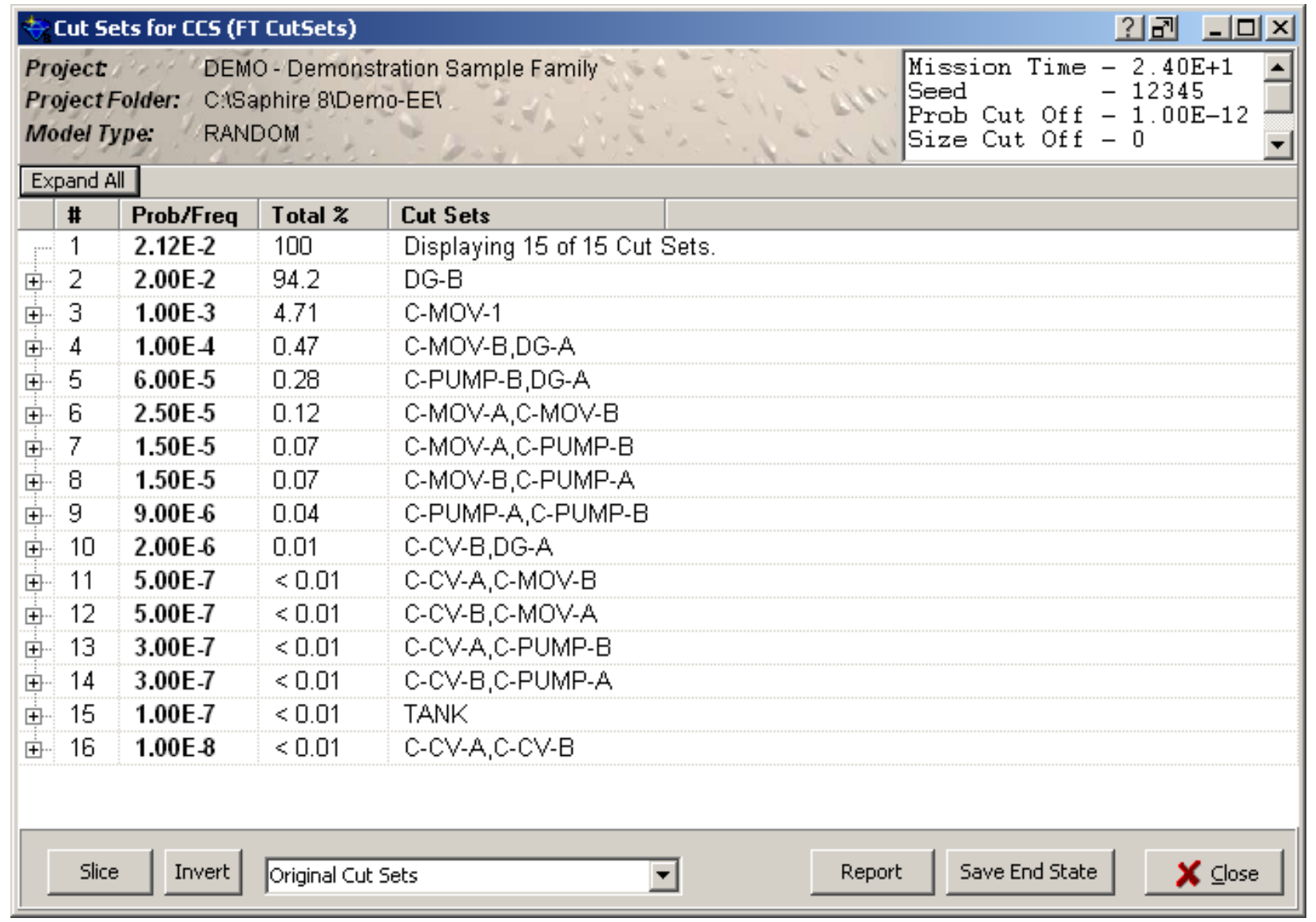

Figure 13. Fault tree cut set viewer.

The other option is to select Publish from the project's main menu bar. This report function allows the user to specify from a default listing of report outputs and select the document structure for printing. 


\begin{tabular}{l}
\hline Edit Logic \\
Edit Properties \\
Edit Cut Set Rules \\
\hline Solve \\
View Summary Results \\
View Cut Sets \\
View Importance Measures \\
\hline View Uncertainty \\
\hline Hide / Show \\
Set Bookmark \\
Goto Bookmark \\
\hline Delete \\
\hline
\end{tabular}

The different types of sampling are available within SAPHIRE 8, Latin Hypercube and Monte Carlo. To use either one of these sampling methods, its radio button must be selected along with the number of samples. The uncertainty option is shown in Figure 14.

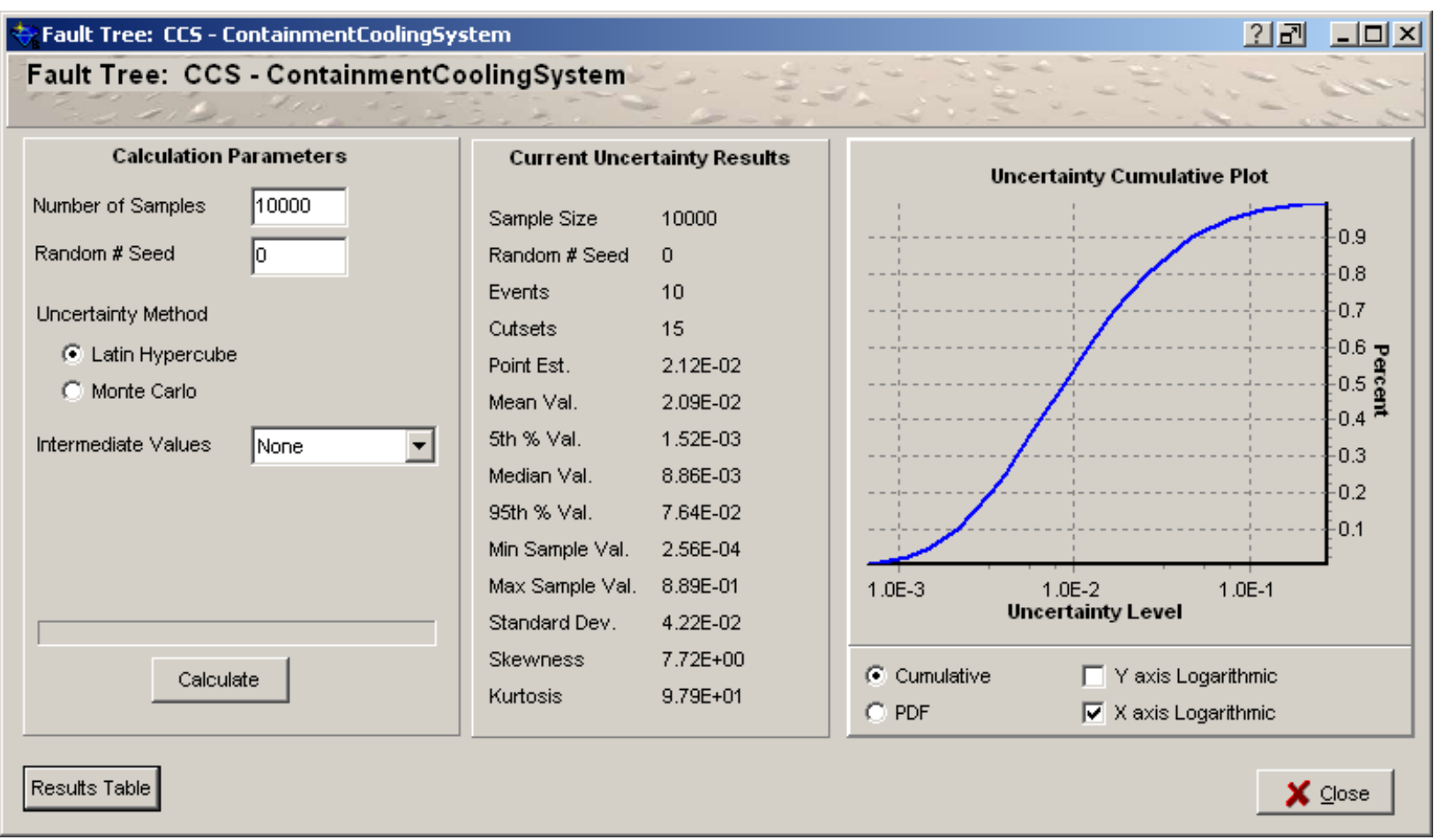

Figure 14. Fault tree uncertainty analysis dialog.

Once the Uncertainty Method is selected then the Calculate button is pressed. SAPHIRE 8 will perform the sampling technique specified for the number of samples. Once SAPHIRE performs the uncertainty analysis it updates the Current Uncertainty Results field and plots the results. 


\section{Importance Measures}

The importance measures in SAPHIRE 8 are the same as in SAPHIRE 7. The only difference is the menu option and the type of outputs. To view the importance measures, highlight the fault tree, right click the mouse and select View Importance Measures (Figure 15).

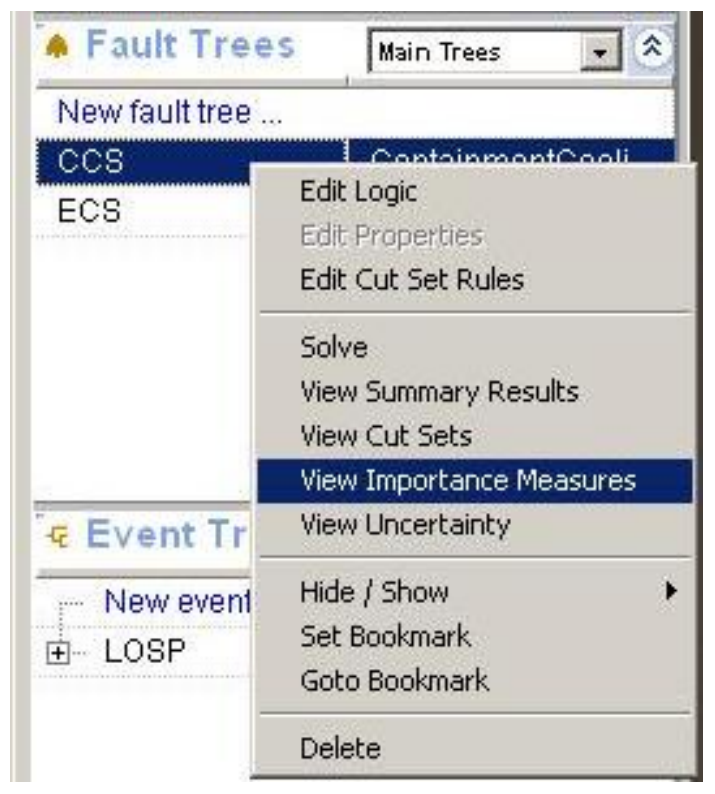

Figure 15. Importance measures path

The Fussell-Vesely (FV) measure is an indication of the percentage of the minimal cut set upper bound contributed by the cut sets containing the basic event. The equation for FV importance now being used (in version 8 ) is

$\mathrm{FV}=\mathrm{F}(\mathrm{i}) / \mathrm{F}(\mathrm{x})$

where

F(i) is the minimal cut set upper bound for the group of cut sets containing the event

$\mathrm{F}(\mathrm{x})$ is the original minimal cut set upper bound

In older versions of SAPHIRE (prior to SAPHIRE version 7.27), the FV equation used an approximate expression

$\mathrm{FV}=1-\mathrm{F}(0) / \mathrm{F}(\mathrm{x})$

where

$\mathrm{F}(0)$ is the minimal cut set upper bound with the event probability set equal to 0.0 .

$\mathrm{F}(\mathrm{x})$ is the original minimal cut set upper bound. 
When basic event probabilities are low, the risk reduction ratio (RRR) and FV measures provide the same ranked list of events. However, when event probabilities become large, the behavior of RRR and FV differ. For example, consider:

System $=$ A “or" B

$\mathrm{P}($ System $)=\mathrm{P}(\mathrm{A})+\mathrm{P}(\mathrm{B})-\mathrm{P}(\mathrm{A}$ and $\mathrm{B})$

When we plot $R R R_{A}$ and $F V_{A}$ as a function of their respective $P()$, where $P(A)=P(B)$, we see the behavior shown in Figure 16. For example, when $\mathrm{P}(\mathrm{A})=\mathrm{P}(\mathrm{B})=0.5$, then:

$\mathrm{FV}_{\mathrm{A}}=0.5 / 0.75=0.66$

Note that had we used the approximate FV found in older versions of SAPHIRE, then:

$\mathrm{FV}_{\mathrm{A}}=1-0.5 / 0.75=0.33$

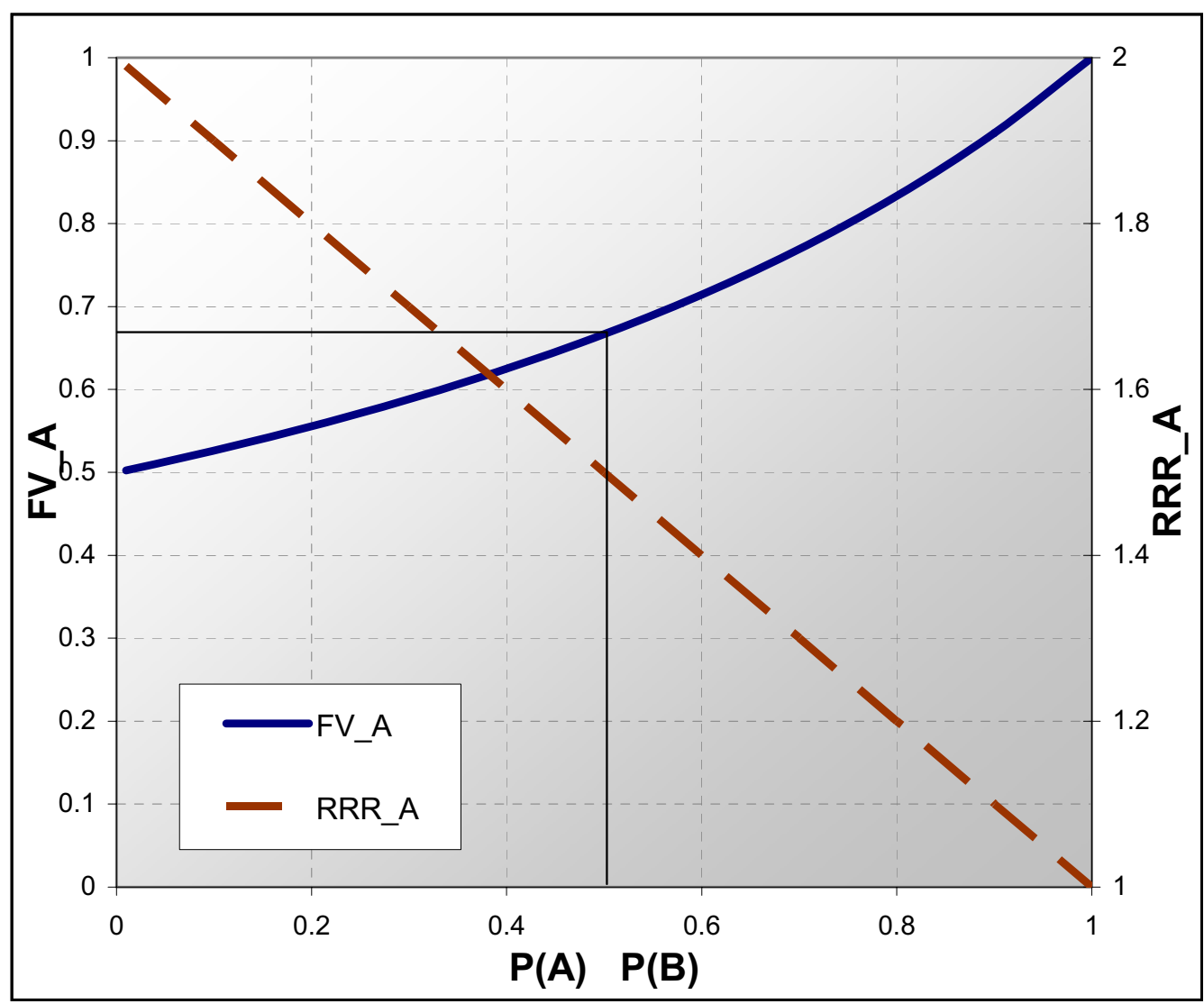

Figure 16. Behavior of FV and RRR as basic event probabilities are varied.

The importance measures are all loaded into one screen for reporting. The importance measures can be reported as point estimates or the component variability can be incorporated into the results. The point estimate is calculated using the formal importance measure equations using just the input probabilities for the individual components and the overall point estimate for the fault tree. However, if the importance of the components based on their variability is required, SAPHIRE 8 will perform the same calculation as 
stated for the point estimate except now it samples from the component's uncertainty and feeds this into the importance measure equation. For example, the standard importance measures are calculated as

$\begin{array}{ll}\text { Fussell-Vesely: } & \mathrm{FV}=\mathrm{F}(\mathrm{i}) / \mathrm{F}(\mathrm{x}) \\ \text { Risk Increase Ratio: } & \mathrm{RIR}=\mathrm{F}(1) / \mathrm{F}(\mathrm{x}) \\ \text { Risk Reduction Ratio: } & \mathrm{RRR}=\mathrm{F}(\mathrm{x}) / \mathrm{F}(0) \\ \text { Birnbaum: } & \mathrm{B}=\mathrm{F}(1)-\mathrm{F}(0)\end{array}$

where: $\mathrm{F}(\mathrm{x})=$ value of all the minimal cut sets evaluated with the basic event probabilities at their mean value.

$\mathrm{F}(\mathrm{i})=$ value of the minimal cut sets that contain the i'th basic event.

$\mathrm{F}(0)=\quad$ value of all the minimal cut sets evaluated with the i'th basic event probability set to zero.

$\mathrm{F}(1)=\quad$ value of all the minimal cut sets evaluated with the i'th basic event probability set to one.

For the point estimate, the equation is solved only once for each component using their input probability. Now to take into consideration the component's variability, the importance measure equation is calculated for each sample. From the results of the samples, the importance measure mean, and other parameters are obtained for each basic event.

The report for the importance measure based on the point estimate is shown in Figure 17. 


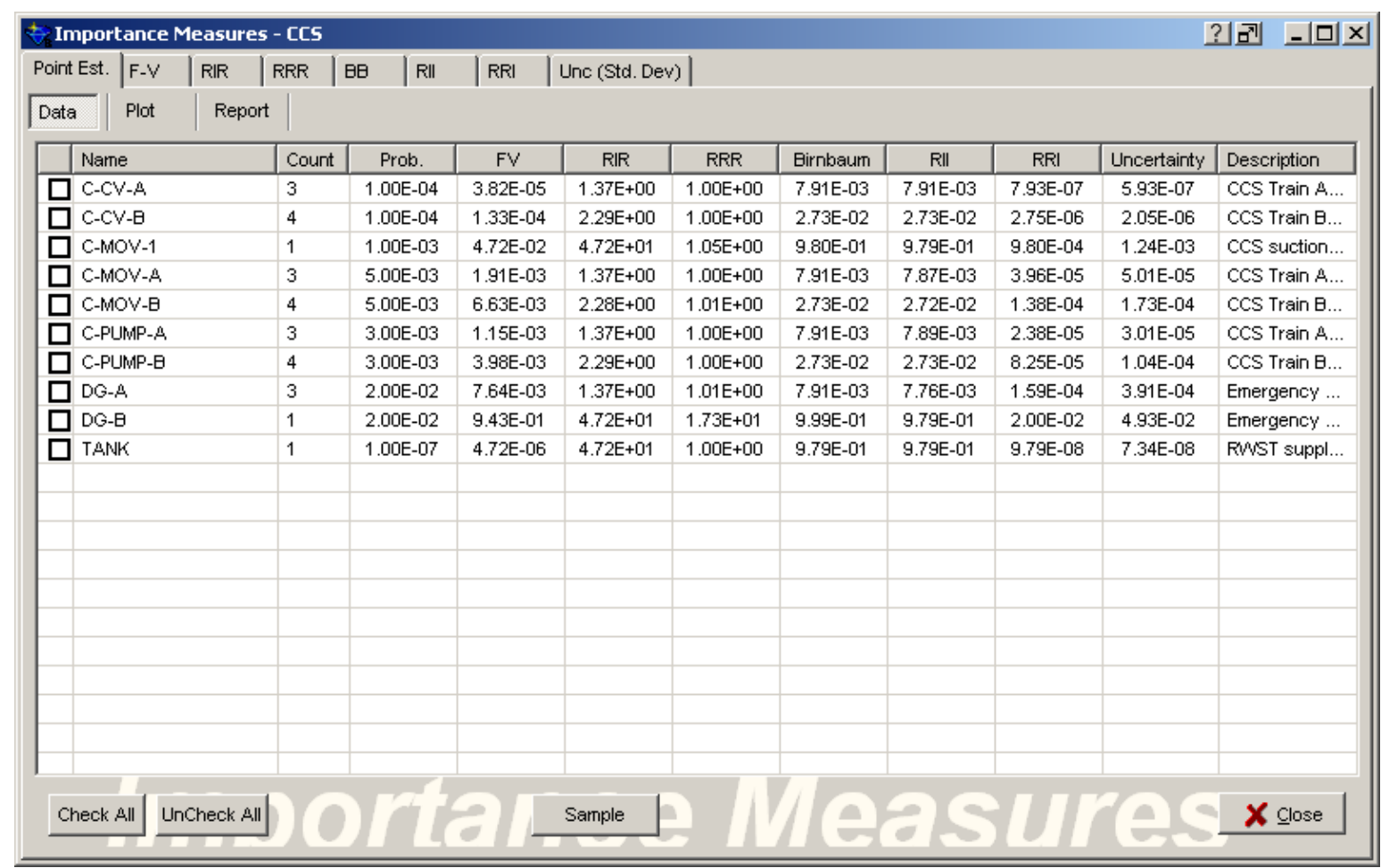

Figure 17. Point estimate importance measure dialog.

The report for the importance measures allowing the component's variability to be propagated through is shown in Figure 18, where the report provides the results of the FV sampling. For each iteration (up to the number of samples specified) SAPHIRE determines the $F(1), F(i), F(0)$, and $F(x)$ terms previously defined, wherein each of the basic events in the cut sets are sampled using their individual epistemic distributions (e.g., lognormal, beta, gamma). Then, for each iteration, the importance measures are quantified using these " $F($ )" terms. Each value for the various importance measures is stored in an array that is later used to determine the moments (such as mean and standard deviation) and percentiles (such as $5^{\text {th }}, 50^{\text {th }}$, and $\left.95^{\text {th }}\right)$.

The calculation arrays are temporarily (due to the potentially large storage space) stored in memory consequently, once the user closes the importance measure screen, the uncertainty results are discarded and must be rerun if needed again. 
\# Importance Measures - CCS

\begin{tabular}{l|l|l|l|l|l|l|l|} 
Point Est. & F-V & RIR & RRR & B日 & Rll & RRI & Unc (Std. Dev)
\end{tabular}

Data | Plot | Report |

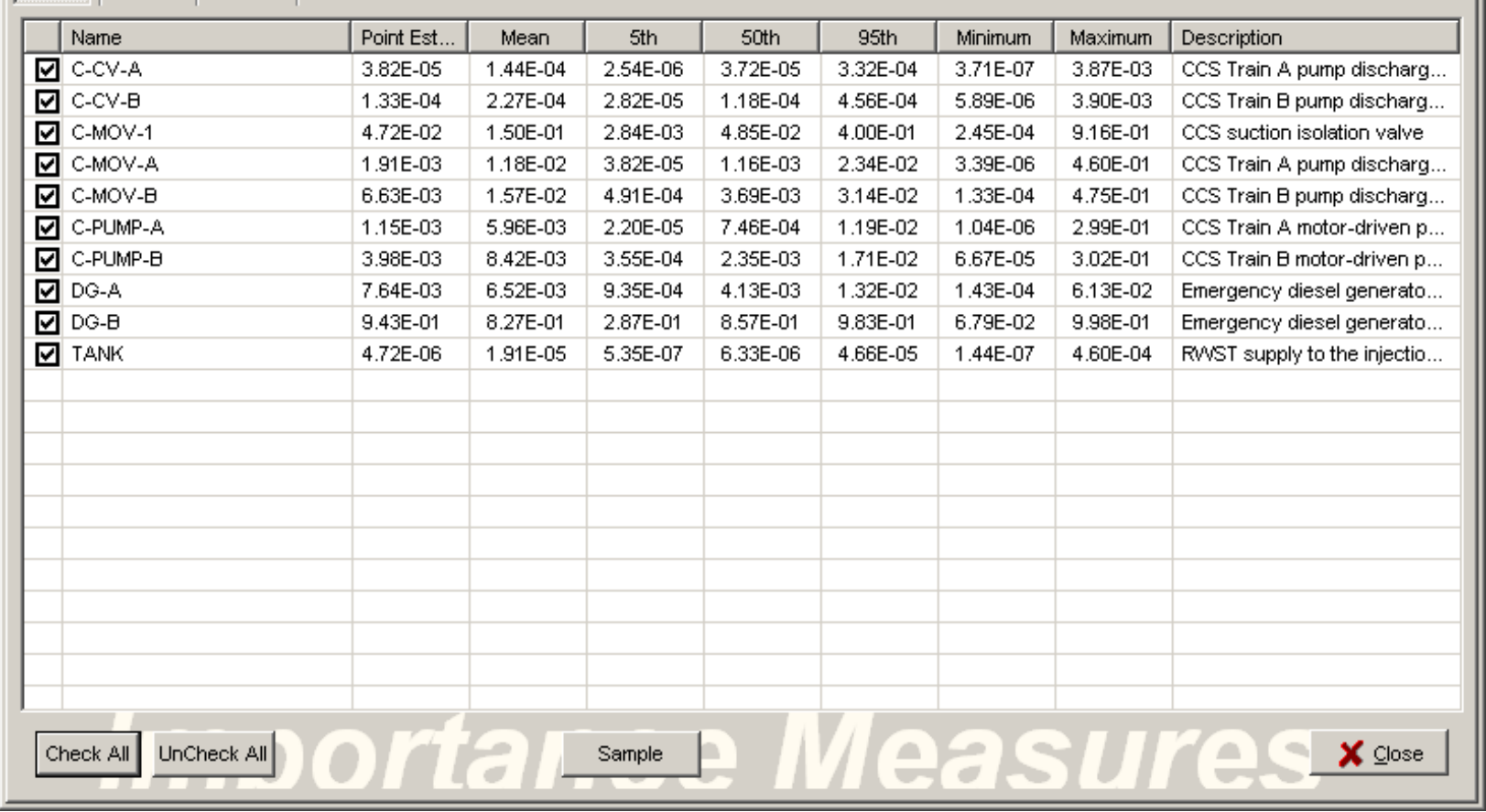

Figure 18. Uncertainty on importance measures.

The other report option for the importance measures is a plot of each component based on the sampling process. This option is displayed by selecting the plot tab as shown in Figure 19. 


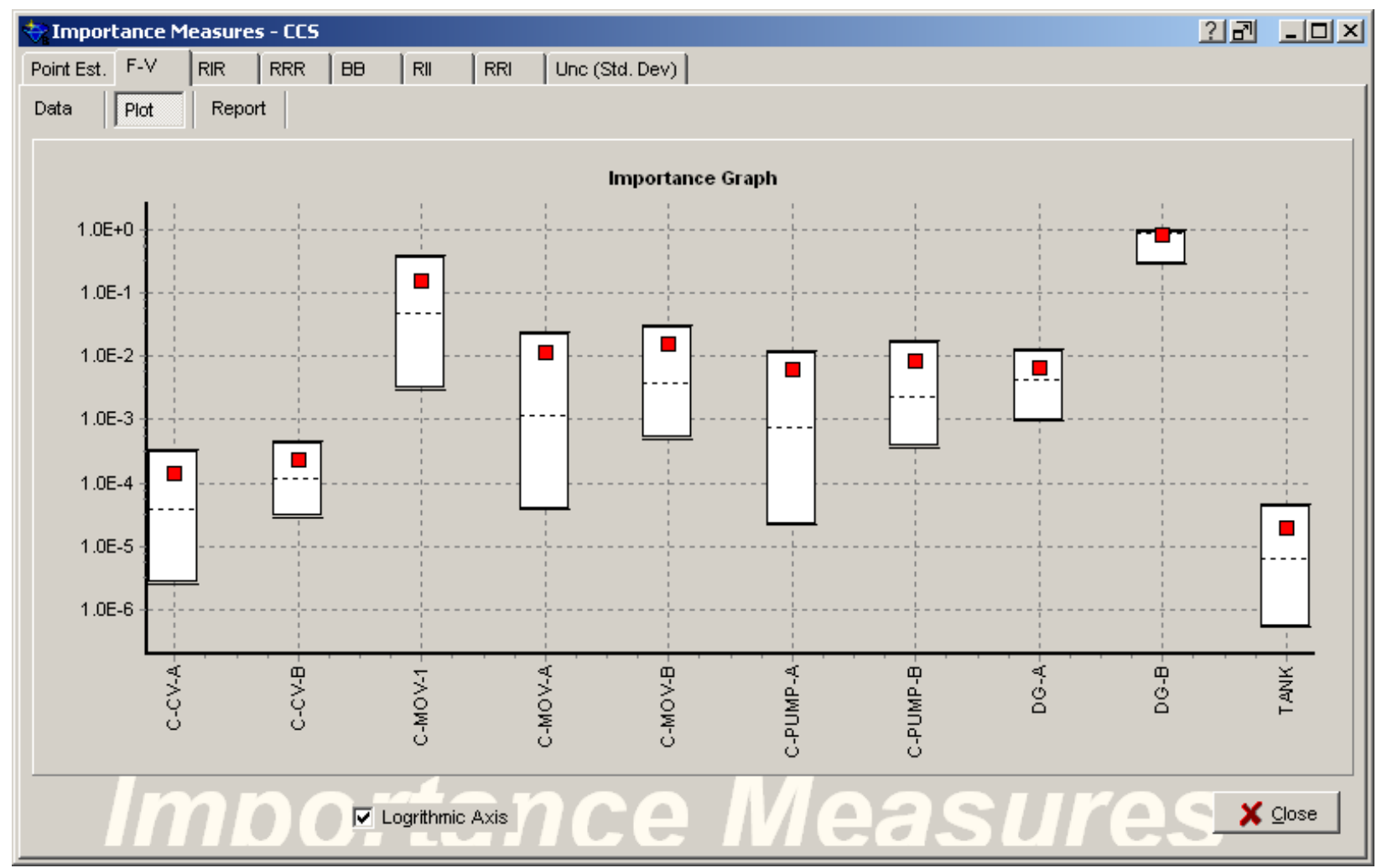

Figure 19. Plotting of importance measure uncertainty.

\section{EVENT TREES}

The analysis process for event trees is designed to solve the accident sequences for minimal cut sets. The cut set generation and other processes are similar to the fault tree option with exception that event tree sequences are used as the logic model. The different aspects of analyzing the event tree accident sequences will be covered

\section{Solve Options}

\section{Cut Set Generation:}

The cut set generation is based on the linked fault tree methodology (i.e. fault tree logic linked to the event tree logic). The solve option (Figure 20) will generate the minimal cut sets for the accident sequence logic, which is then quantified. Depending if the event tree is highlighted or an individual sequence will determine which sequences are analyzed. 


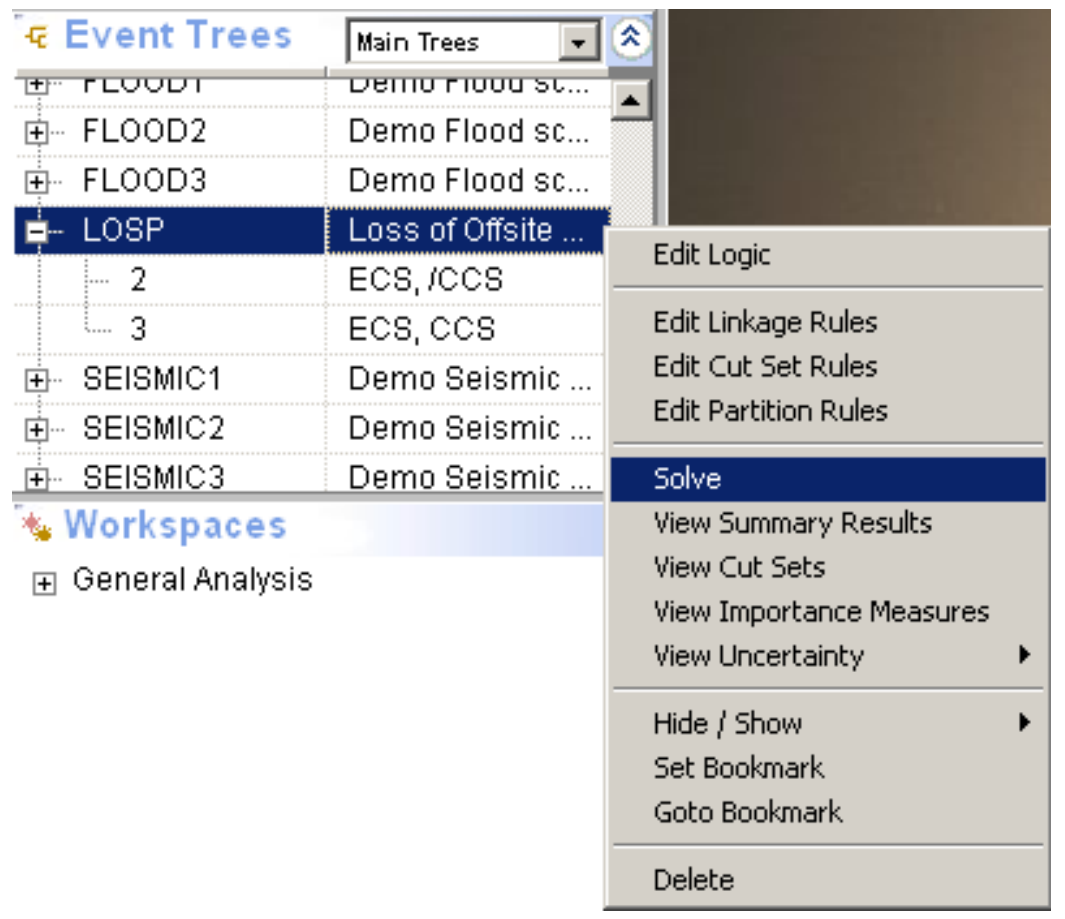

Figure 20. Event Tree Cut Set solve option path

Once the Solve option has been selected, SAPHIRE will pop up a screen that allows for sequence analysis settings. Each of the options will be discussed.

\section{Cut Set Truncation:}

None - This truncation option solves for all possible cut sets.

Normal - This truncation option generates all cut sets above the truncation value specified divided by the initiating event frequency (i.e., truncation value specified/initiating event frequency).

Conditional - This truncation option generates all cut sets above the truncation value specified regardless of the initiating event frequency.

\section{Solution Steps:}

The solution steps check boxes are designed to specify how to solve the accident sequences. These check boxes can be checked individually and the Solve button clicked or multiple boxes can be checked and SAPHIRE will apply all operations that are checked starting at top and working down.

Solve for Cut Sets - This check box will solve all event tree accident sequences that are highlighted for their minimal cut sets based on the sequence logic.

Apply Recovery Rules - This check box will apply all post-processing rules to the newly generated cut sets (Sequence specific rule, Event Tree specific rule, then Project rule).

Update/Quantify Cut Sets - This option re-evaluates the existing cuts to remove any potential non-minimal cut sets and then quantifies these cut sets using the default calculation.

Clear Cut Set Partitions - This option resets the partition rules (i.e., removes the end states from the cut sets that was created using partition rules). 
Apply Partition Rules - This option applies the partition rules to create user specific end states for the cut sets based on rules (Sequence specific rule, Event Tree specific rule, then Project rule). Update Base Case - This option stores the resultant cut sets into the base case.

\section{Model Types:}

The last option within this screen is what Model Types are to be solved. Each basic event/fault tree/event tree can be susceptible to one or more of the Model Types available within SAPHIRE. The default is Random, but multiple Model Types can be selected and SAPHIRE will solve the fault tree based on the Model Type(s) selected (Figure 21). The results will all be grouped together based on the Model Type(s) selected.

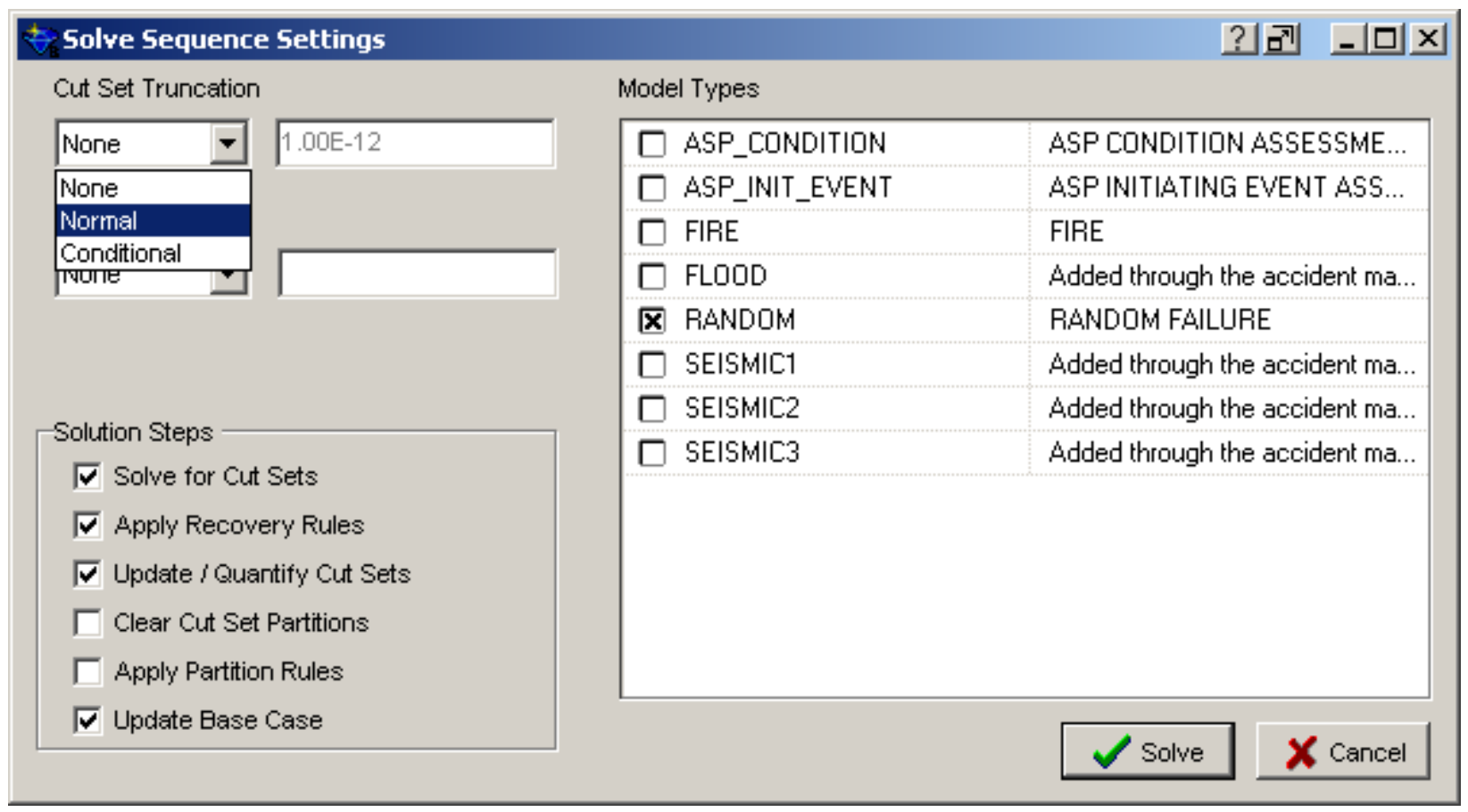

Figure 21. Model type selection for solving sequences

\section{Report:}

There are multiple Report options, just like in SAPHIRE 7. The two options from within the event tree menu field are View Summary Results and View Cut Sets (Figure 22). 


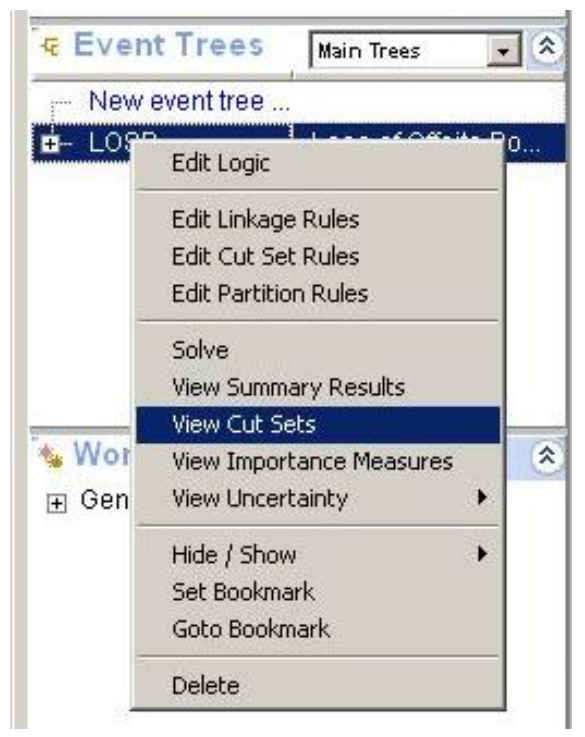

Figure 22. Event Tree View Cut Set path

Once the event tree accident sequence(s) have been solved then the cut sets can be viewed. From the screen below (Figure 23) the accident sequence cut sets can be reported by selecting the Report button. This option will print out the resultant cut sets for preview and then to the selected printer. 


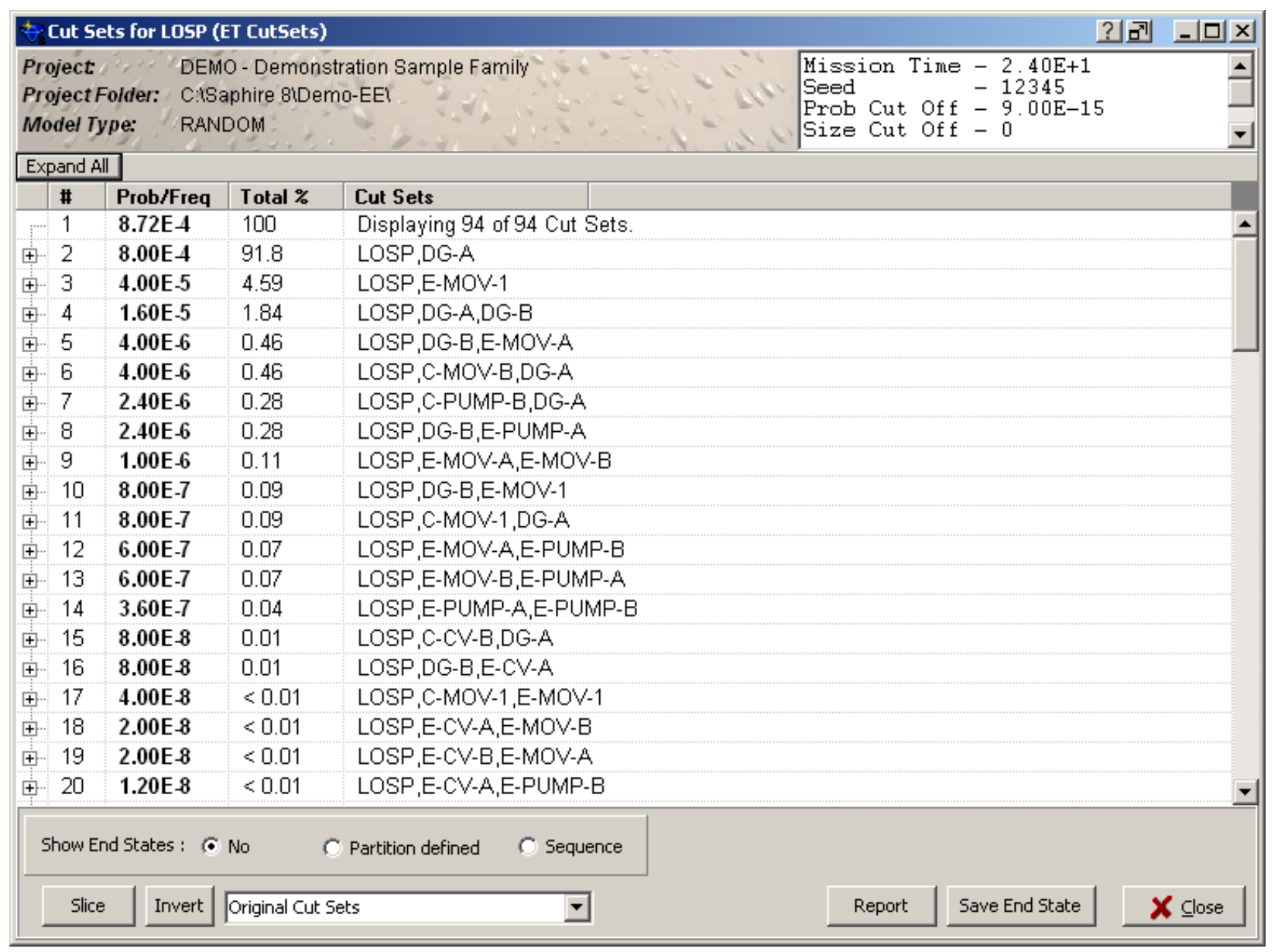

Figure 23. Cut Sets view window

The other option is to select Publish from the project's main menu bar. This report function allows the user to specify from a default listing of report outputs and select the document structure for printing.

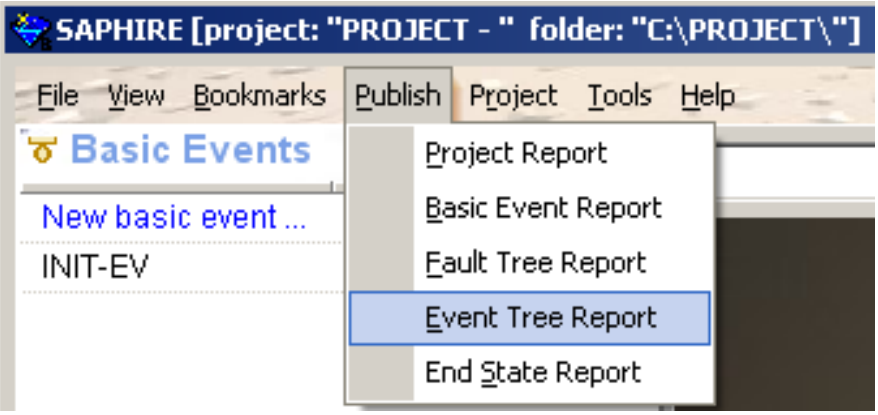

Figure 24. Event Tree Report path

\begin{tabular}{|c|c|c|}
\hline - & Main Trees & + 숫 \\
\hline \multicolumn{3}{|l|}{ New event tree ... } \\
\hline$\dagger \cdots$ ET1 & Event Tree 1 & \\
\hline$+\cdots$ ET2 & Event Tree 2 & \\
\hline$+-\mathrm{ET3}$ & Event Tree 3 & \\
\hline$+\cdots$ ET4 & Event Tree 4 & \\
\hline †... ET5 & Event Tree 5 & \\
\hline
\end{tabular}




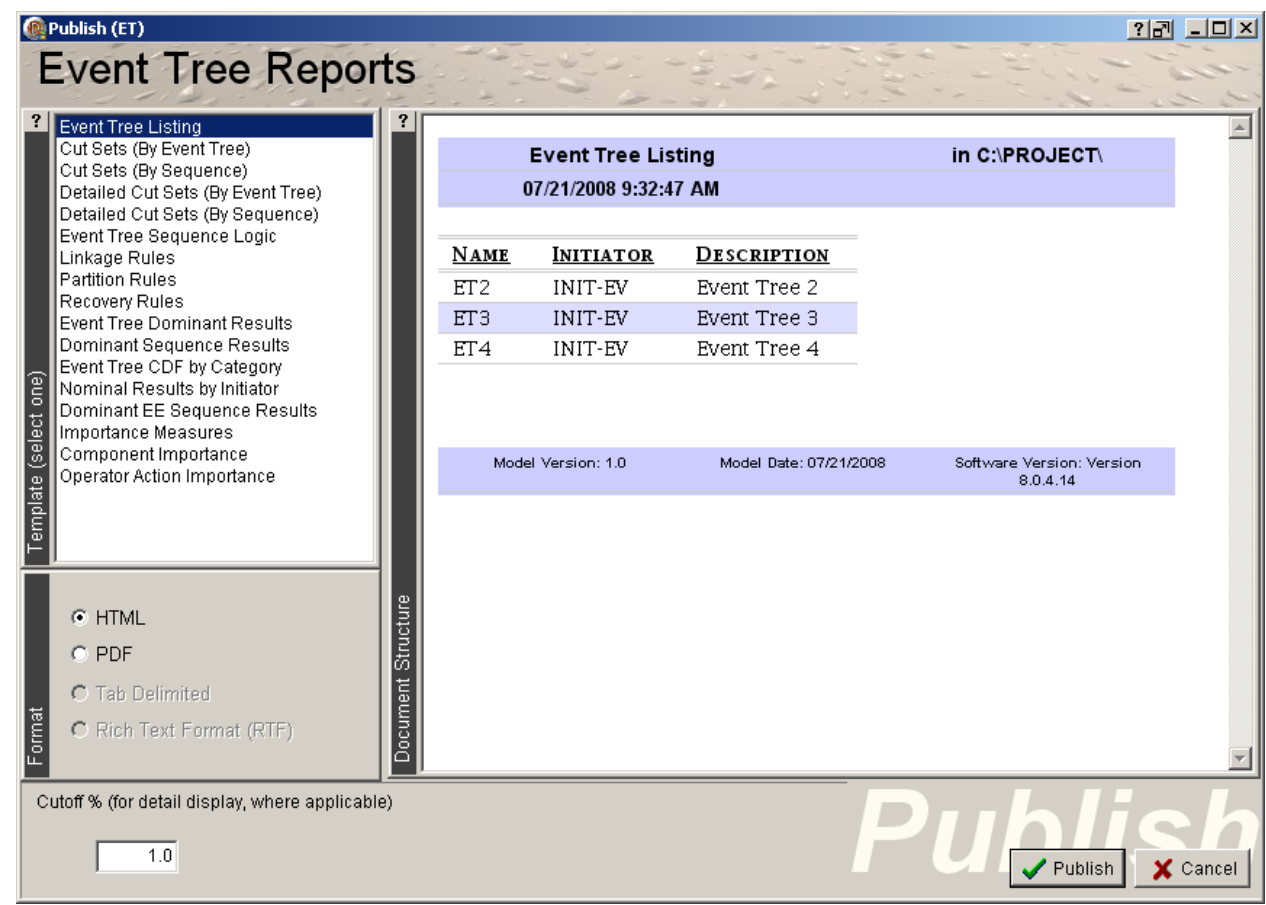

Figure 25. Event Tree Report

\section{Uncertainty Analysis}

The Uncertainty analysis is the same as that performed in SAPHIRE 7. The only difference is the menu options and improved plotting. In SAPHIRE 8, the Uncertainty analysis is performed in the View Uncertainty option after the cut sets have been generated. The type of uncertainty needs to be specified on whether all highlighted sequences are to be analyzed independently or if all highlighted sequences are to be analyzed as a group.

The different types of sampling available within SAPHIRE 8 are Latin Hypercube and Monte Carlo. To use either one of these sampling methods, its radio button must be selected along with the number of samples. The Uncertainty option shown below is for all highlighted sequences to be analyzed individually to obtain a single result for each sequence. 


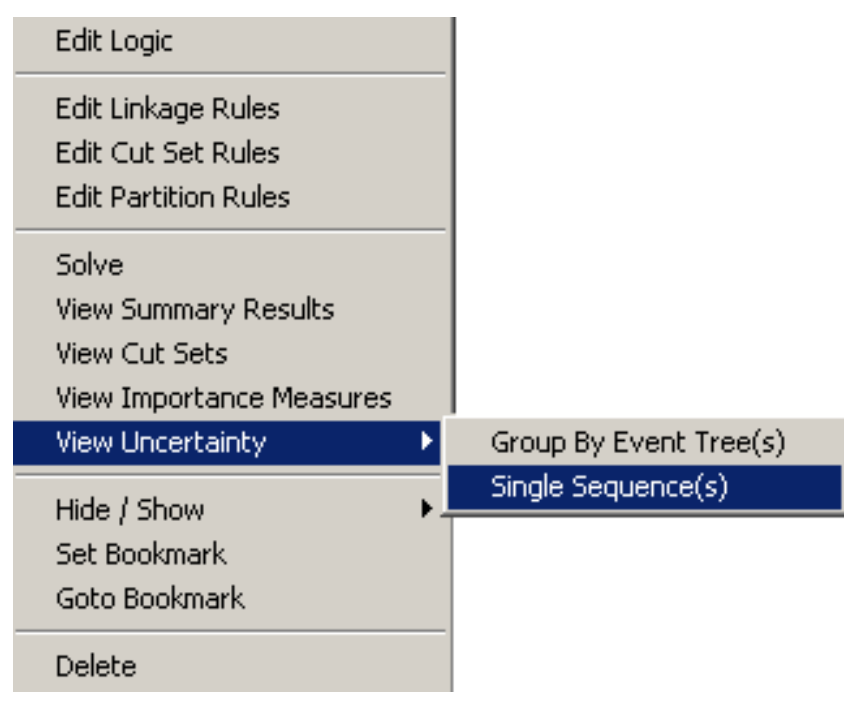

Figure 26. Uncertainty Analysis path

Once the Uncertainty Method is selected then the Calculate button is pressed. SAPHIRE 8 will perform the sampling technique specified for the number of samples. Once SAPHIRE performs the uncertainty analysis it plots the results (Figure 27). The percentiles and overall mean can be displayed by selecting the Results Table button.

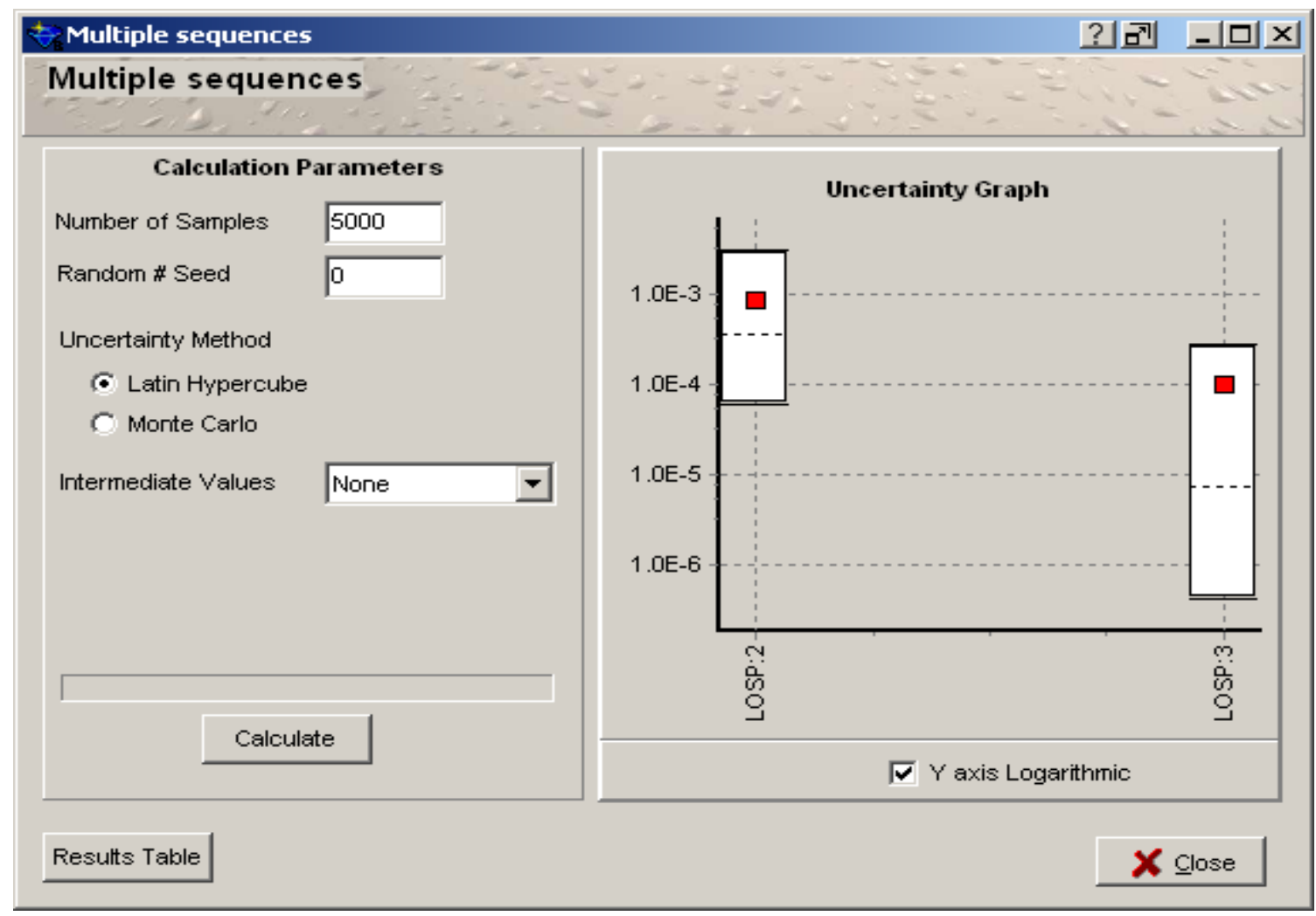

Figure 27. Uncertainty plot example 


\section{Importance Measures}

The importance measures in SAPHIRE 8 are the same as in SAPHIRE 7. The only difference is the menu option and the type of outputs. To view the importance measures, highlight the fault tree, right click the mouse and select View Importance Measures.

\begin{tabular}{l} 
Edit Logic \\
\hline Edit Linkage Rules \\
Edit Cut Set Rules \\
Edit Partition Rules \\
\hline Solve \\
View Summary Results \\
View Cut Sets \\
\hline View Importance Measures \\
\hline View Uncertainty \\
\hline Hide / Show \\
Set Bookmark \\
Goto Bookmark \\
\hline Delete
\end{tabular}

Figure 28. View Importance Measures path

The importance measures are all loaded into one screen for reporting. The importance measures can be reported as point estimates or the component variability can be incorporated into the results. The point estimate is calculated using the formal importance measure equations using just the input probabilities for the individual components and the overall point estimate for the accident sequence(s). However, if the importance of the components based on their variability is required, SAPHIRE 8 will perform the same calculation as stated for the point estimate except now it samples from the component's uncertainty and feeds this into the importance measure equation. For example, Fussell-Vesely (FV) is calculated as

$\mathrm{FV}_{\mathrm{i}}=\mathrm{F}_{\mathrm{i}}(\mathrm{x}) / \mathrm{F}(\mathrm{x})$

where: $\mathrm{F}(\mathrm{x})$ is the original minimal cut set upper bound

$\mathrm{F}_{\mathrm{i}}(\mathrm{x})$ is the minimal cut set upper bound with only the basic event of interest.

For the point estimate, the equation is solved only once for each component using their input probability. Now to take into consideration the component's variability, the FV equation is calculated for each sample. From the results of the samples, the FV mean, and other parameters are obtained for each component.

The report for the importance measure based on the point estimate is shown in Figure 29. 


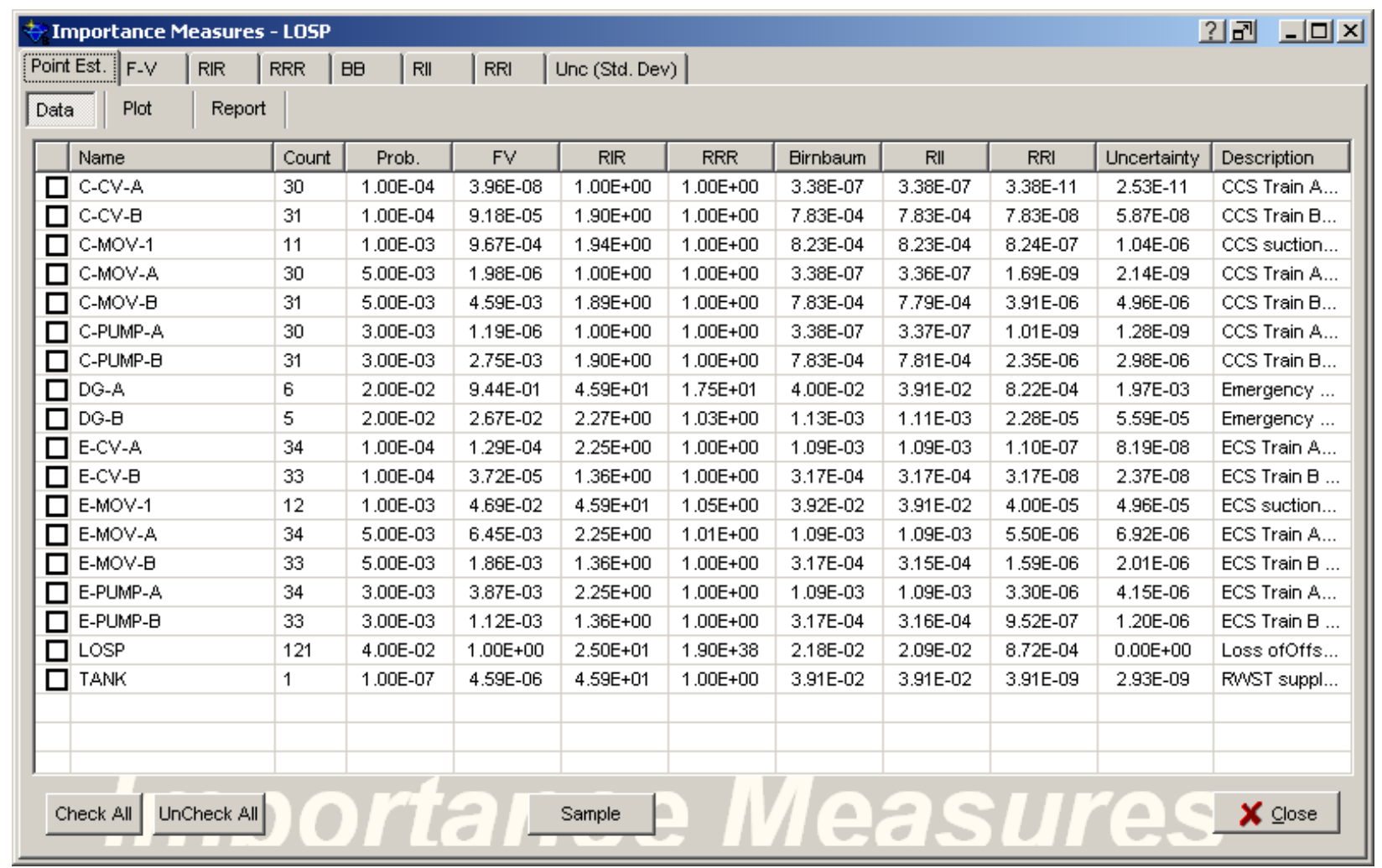

Figure 29. Importance Measures based on point estimate

The report for the importance measures allowing the component's variability to be propagated through is listed below. The report shown below provides the results of the FV sampling (Figure 30). 


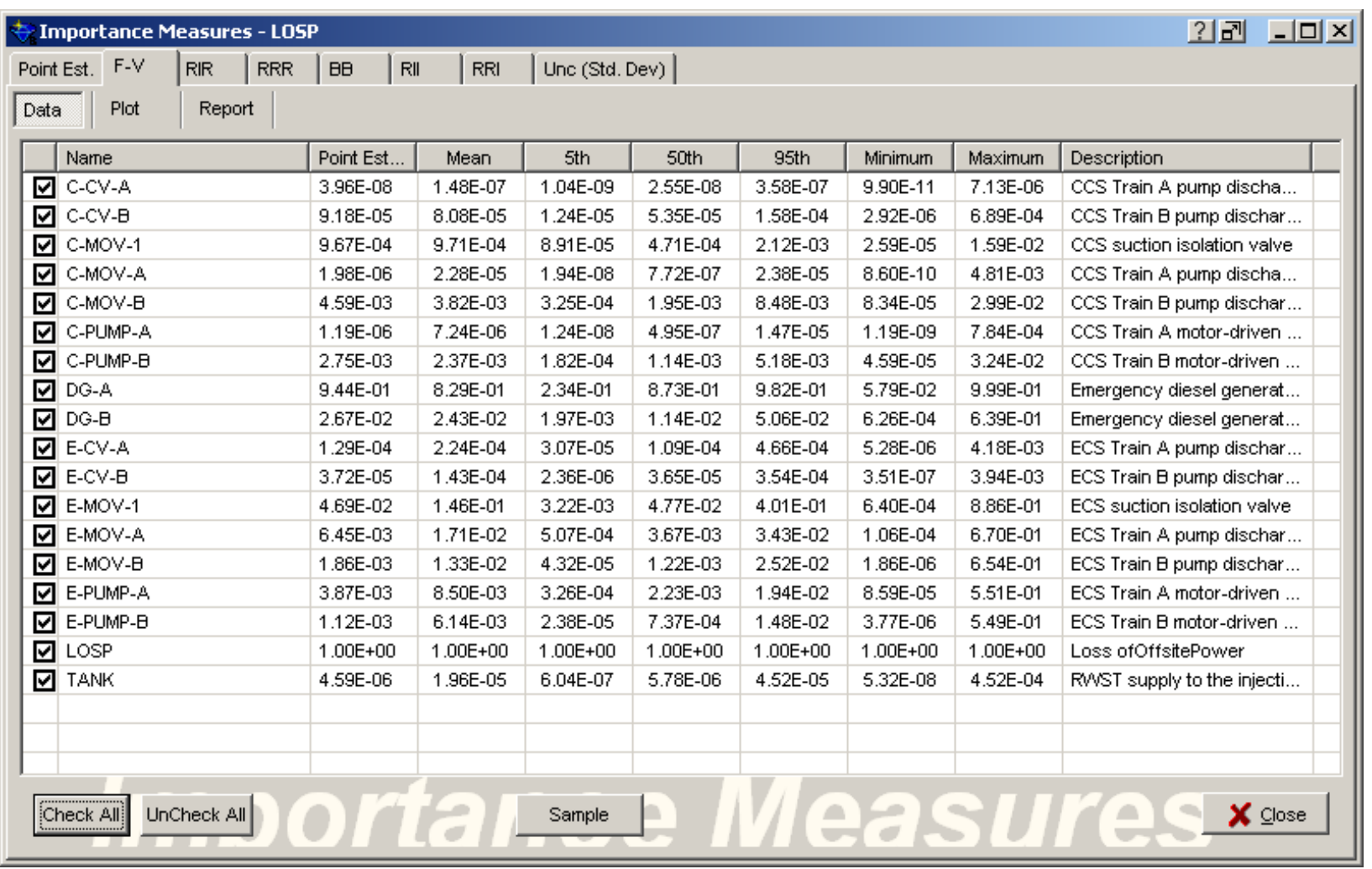

Figure 30. FV Importance Measures report

The other report option for the importance measures is a plot of each component based on the sampling process and this plot is shown in Figure 31. In this plot, the $5^{\text {th }}$ and $95^{\text {th }}$ percentiles are shown via the white box, while the mean is shown as a small red box. 


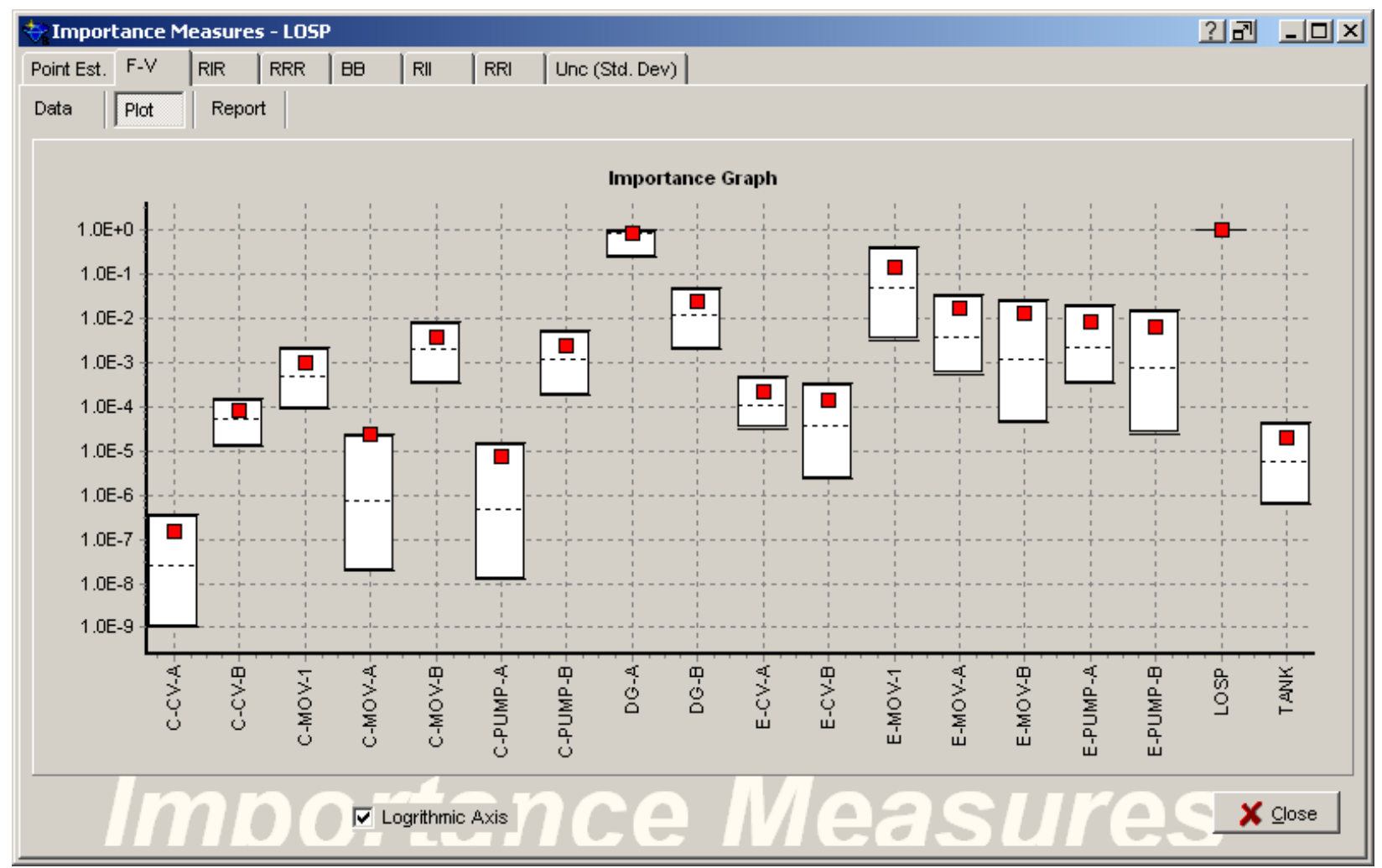

Figure 31. Plotting importance measure uncertainty.

\section{RASP COMMON-CAUSE FAILURE MODULE}

This CCF module uses Boolean reduction to realize combinations of CCF basic events, and removes approximations made in the conditional CCF probability modeling for events assessment as a result of setting components' failure modes to $1, \mathrm{~T}, 0$, or F.

This common cause method is a new calculation type. The common cause failure type " $\mathrm{R}$ " can be found in the basic events under the "Failure Model" option (as seen in Figure 32). 


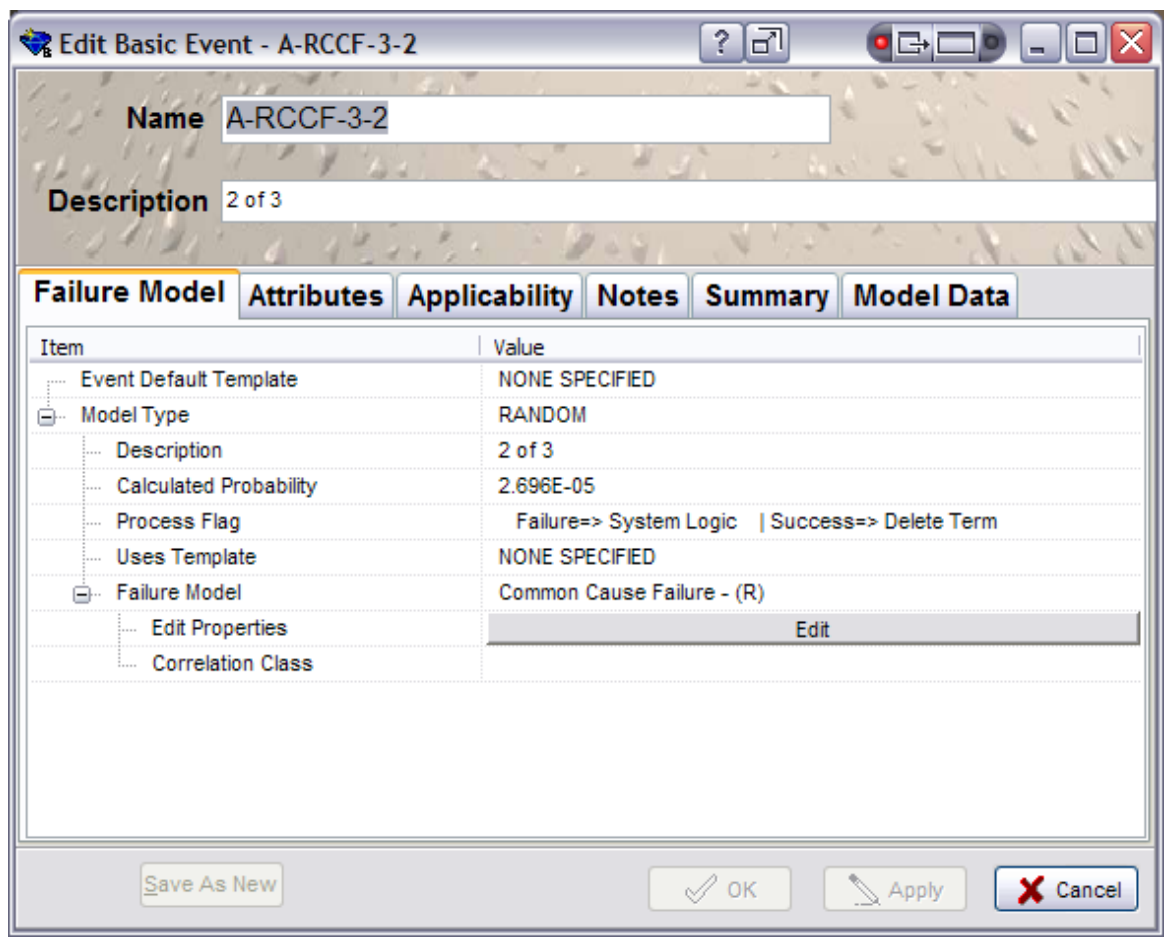

Figure 32. A basic event set up as the new CCF type.

To demonstrate the CCF module, a new CCF object will be created. A system will be modeled of three redundant motor operated valves (MOVs), represented by basic events MOV-CC-A, MOV-CC-B, and MOV-CC-C. The success criterion for this system is that two components have to work for the system to work. Consequently, if two (of three) components fail, the system is failed.

The basic event representing CCF will be called MY_CCF. The fault tree representing the system is shown below. 


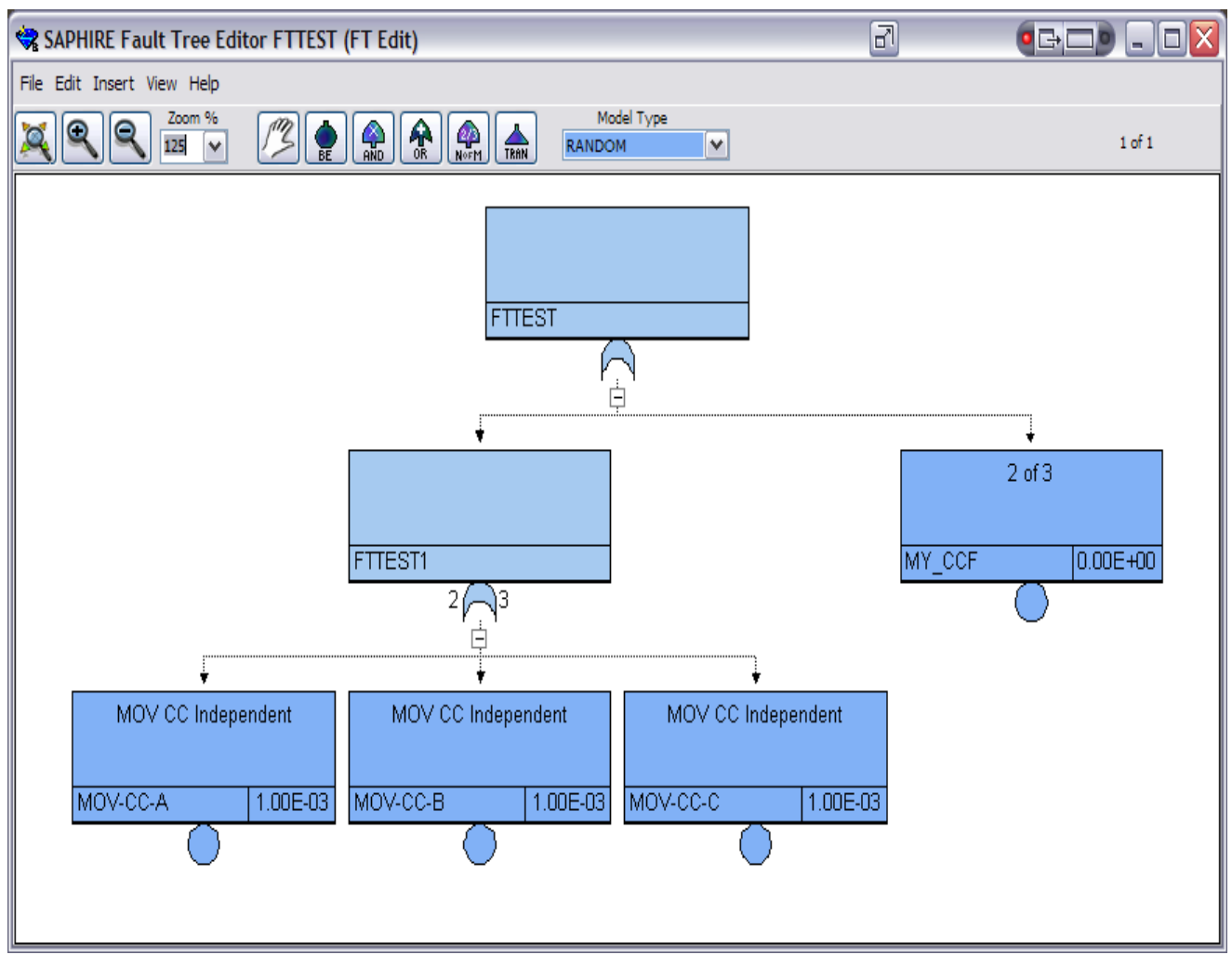

Figure 33. Fault tree with CCF representing the example system.

The basic event MY_CCF is a part of the fault tree graphic as shown in Figure 33. However, MY_CCF is a basic event and must be edited. 


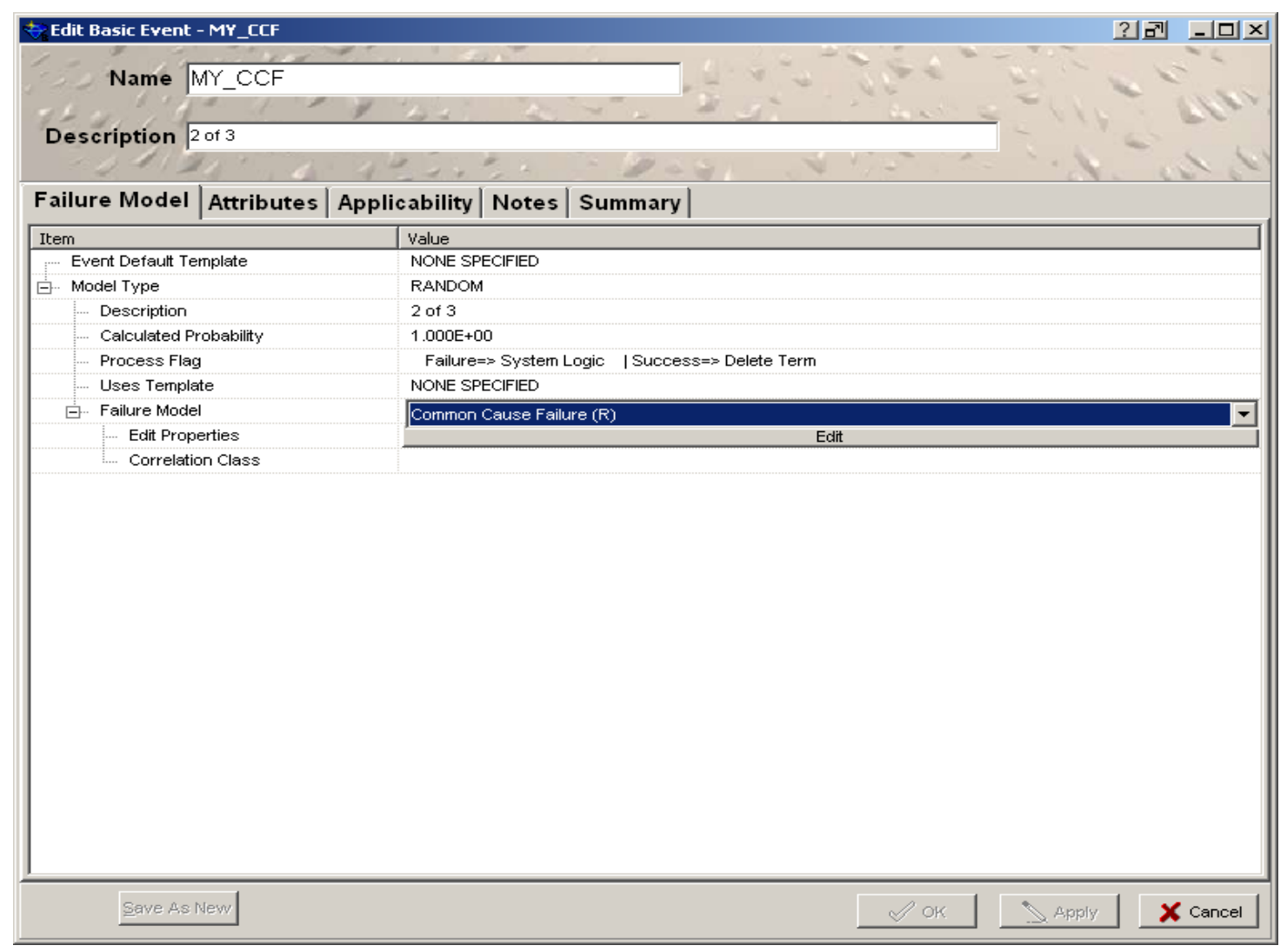

Figure 34. Editing the example CCF basic event.

To make a CCF object, its calculation type must be set to "Common Cause Failure - (R)." Once this calculation type is selected, the user can edit the parameters of the CCF object by clicking the "Edit" button or the "Model Data" tab. The Model Data for CCF objects appears as shown below. For the example case, the three Independent Failure Events (MOV-CC-A, MOV-CC-B, and MOV-CC-C) and the CCF Factors need to be specified. Since the alpha-factor model was selected, the Factors asked by SAPHIRE are alpha, alpha $_{2}$, and alpha 3 (labeled Alpha 1, Alpha 2, and Alpha 3, respectively). These Factors are other basic events that must already exist in the database.

For this example, a "staggered" testing scheme is assumed. As already indicated, the Failure Criteria is set to "2."

The Separator character is used by SAPHIRE to determine the "auto generated" event names, as we will see when describing the results of the CCF calculation. Initially, the default results are given as "Rolledup," meaning the overall CCF probability is returned by this object. In this example, the overall CCF probability is 2.696E-5. However, the details of this calculation can be seen by changing the "Results Detail Level" to "Full Detail." 


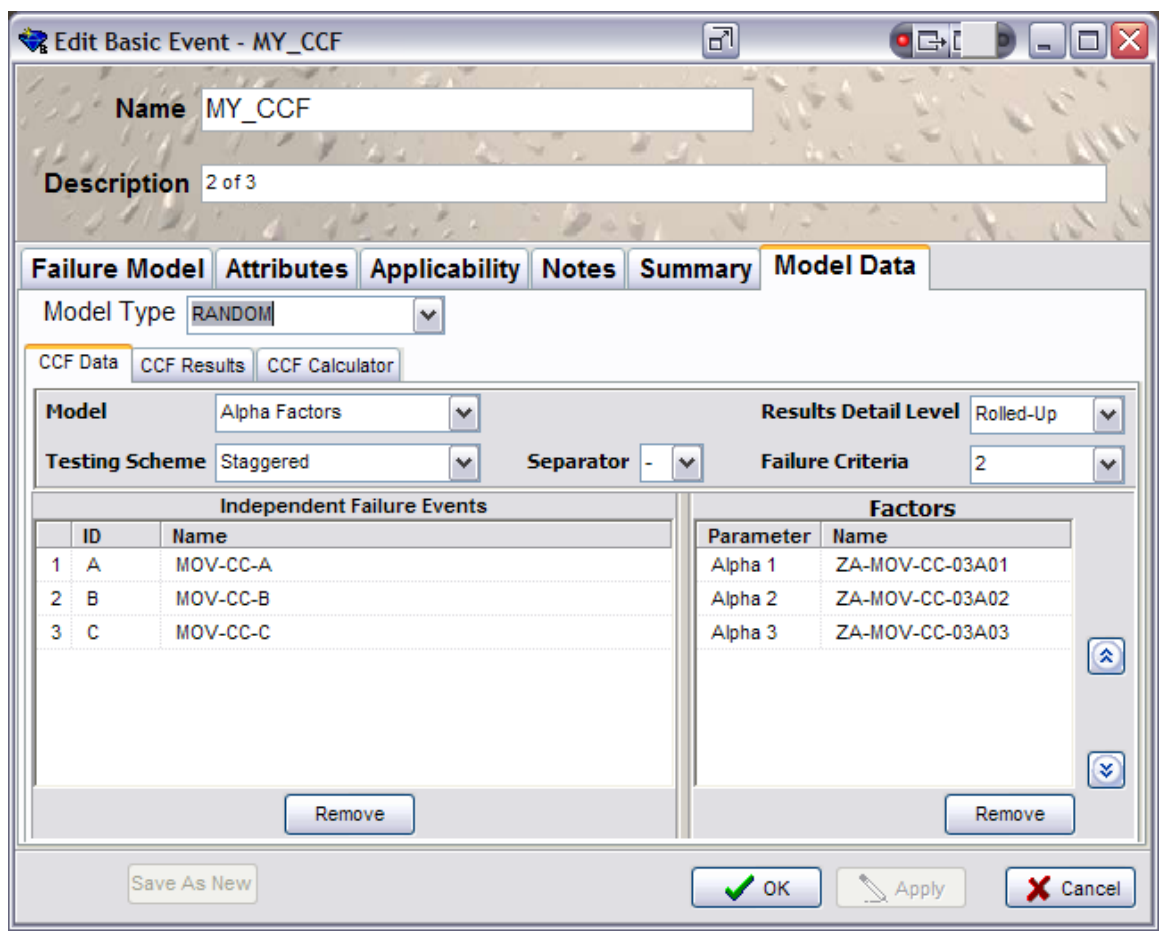

Figure 35. Editing the parameters of the CCF object.

When evaluating the "Full Detail" results for this example, the results can be seen by clicking on the "CCF Results" tab. The detailed results appear as:

2 inputs out of 3 possible must fail - All independent only groups are not counted.

\# 1, 6.4500E-06, MY_CCF-AB

\# 2, 6.4500E-06, MY_CCF-AC

\# 3, 6.4500E-06, MY_CCF-BC

\# 4, 7.6100E-06, MY_CCF-ABC

Q1 $=9.7950 \mathrm{E}-04$

$\mathrm{Q} 2=6.4500 \mathrm{E}-06$

$\mathrm{Q} 3=7.6100 \mathrm{E}-06$

$3{ }^{*} \mathrm{Q} 2$

$1{ }^{*} \mathrm{Q} 3$

4 permutations.

$2.6960 \mathrm{E}-05$ total failure value.

If these results are evaluated in more detail, the following are seen: 
2 inputs out of 3 possible must fail - All independent only groups are not counted.

This line indicates that two of three components have to fail to have failure of this part of the fault tree. Also, that a term with ALL independent failures are not counted in the CCF probability.

Next, it is seen:

$$
\begin{aligned}
& \text { \# 1, 6.4500E-06, MY_CCF-AB } \\
& \text { \# 2, 6.4500E-06, MY_CCF-AC } \\
& \text { \# 3, 6.4500E-06, MY_CCF-BC } \\
& \text { \# 4, 7.6100E-06, MY_CCF-ABC }
\end{aligned}
$$

These lines represent the "cut sets" included in the CCF calculation. The first three cut sets represent a $\mathrm{CCF}$ of two components, specifically $\mathrm{AB}$ or $\mathrm{AC}$ or $\mathrm{BC}$. The last line represents $\mathrm{CCF}$ of all three components. Their respective probabilities are also listed, for example the probability of seeing A and B fail due to CCF is 6.45E-6. Also note the name of the CCF "events" listed here. For A and B failing due to CCF, SAPHIRE automatically creates the name "MY_CCF-AB" by appending the CCF basic event name with the separator character ("-") and then the identifier for the A and B components (A and B, respectively).

Next, it is seen:

$$
\begin{aligned}
& \mathrm{Q} 1=9.7950 \mathrm{E}-04 \\
& \mathrm{Q} 2=6.4500 \mathrm{E}-06 \\
& \mathrm{Q} 3=7.6100 \mathrm{E}-06
\end{aligned}
$$

These lines represent the $\mathrm{Q}_{1}, \mathrm{Q}_{2}$, and $\mathrm{Q}_{3}$ terms in the CCF basic parameter model used by the calculation. Next, it is seen:

$$
\begin{aligned}
& 3^{*} \mathrm{Q} 2 \\
& 1^{*} \mathrm{Q} 3
\end{aligned}
$$

These lines indicate that we see three combinations of $\mathrm{Q}_{2}$ terms, and only one $\mathrm{Q}_{3}$ term. Consequently, for this example, the full expression to determine the CCF probability is given by:

$\mathrm{P}(\mathrm{CCF})=3 * \mathrm{Q}_{2}+\mathrm{Q}_{3}=3 * 6.45 \mathrm{E}-6+7.61 \mathrm{E}-6=2.696 \mathrm{E}-5$.

Next, a line is seen indicating how many CCF terms we are accounting for in the CCF calculation.

\section{4 permutations.}

Lastly, the CCF probability is seen. 
Note that if MY_CCF is set to have results "Rolled up" then only a basic event with probability of 2.696E-5 will appear in the fault tree cut sets, or:

\begin{tabular}{llll}
\hline \hline$\#$ & Prob/ Freq & Total\% & Cut Set \\
\hline \hline 1 & $2.696 \mathrm{E}-5$ & 89.99 & MY_CCF \\
\hline 2 & $1.000 \mathrm{E}-6$ & 3.34 & MOV-CC-A, MOV-CC-B \\
\hline 3 & $1.000 \mathrm{E}-6$ & 3.34 & MOV-CC-A, MOV-CC-C \\
\hline 4 & $1.000 \mathrm{E}-6$ & 3.34 & MOV-CC-B, MOV-CC-C \\
\hline Total & $2.996 \mathrm{E}-5$ & & \\
\hline
\end{tabular}

However, if detailed results are allowed to appear, the results will show all of the combinations of the CCF terms, or:

\begin{tabular}{llll}
\hline \hline$\#$ & Prob/Freq & Total\% & Cut Set \\
\hline \hline 4 & $7.610 \mathrm{E}-6$ & 25.40 & MY_CCF-ABC \\
\hline 1 & $6.450 \mathrm{E}-6$ & 21.53 & MY_CCF-AB \\
\hline 2 & $6.450 \mathrm{E}-6$ & 21.53 & MY_CCF-AC \\
\hline 3 & $6.450 \mathrm{E}-6$ & 21.53 & MY_CCF-BC \\
\hline 5 & $1.000 \mathrm{E}-6$ & 3.34 & MOV-CC-A, MOV-CC-B \\
\hline 6 & $1.000 \mathrm{E}-6$ & 3.34 & MOV-CC-A, MOV-CC-C \\
\hline 7 & $1.000 \mathrm{E}-6$ & 3.34 & MOV-CC-B, MOV-CC-C \\
\hline Total & $2.996 \mathrm{E}-5$ & &
\end{tabular}

By having all of the detail from the CCF, the fault tree calculation is equivalent to having the fault tree logic as shown in Figure 36 and Figure 37:

In later versions of SAPHIRE 8, the "R" CCF events will be adjusted for sequences where flag sets are used. 


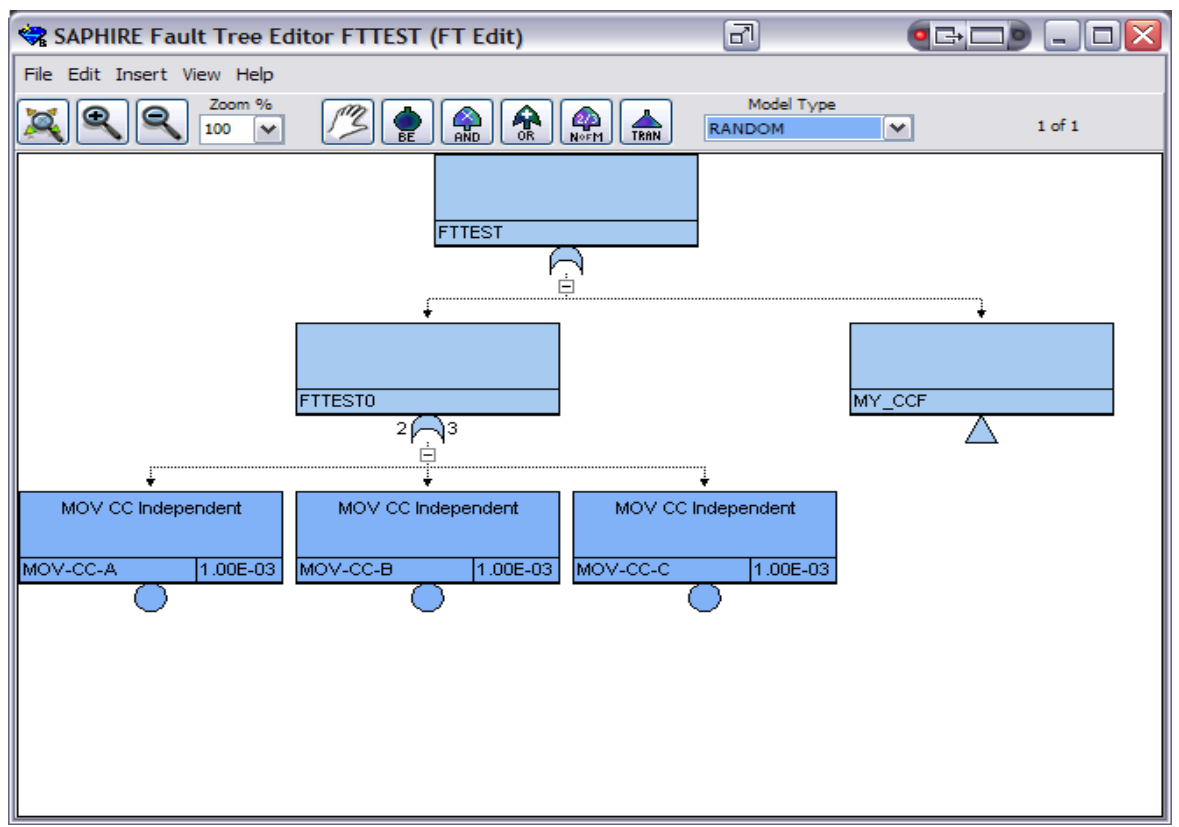

Figure 36. Fault Tree with MOV CCs independent and MY_CCF included

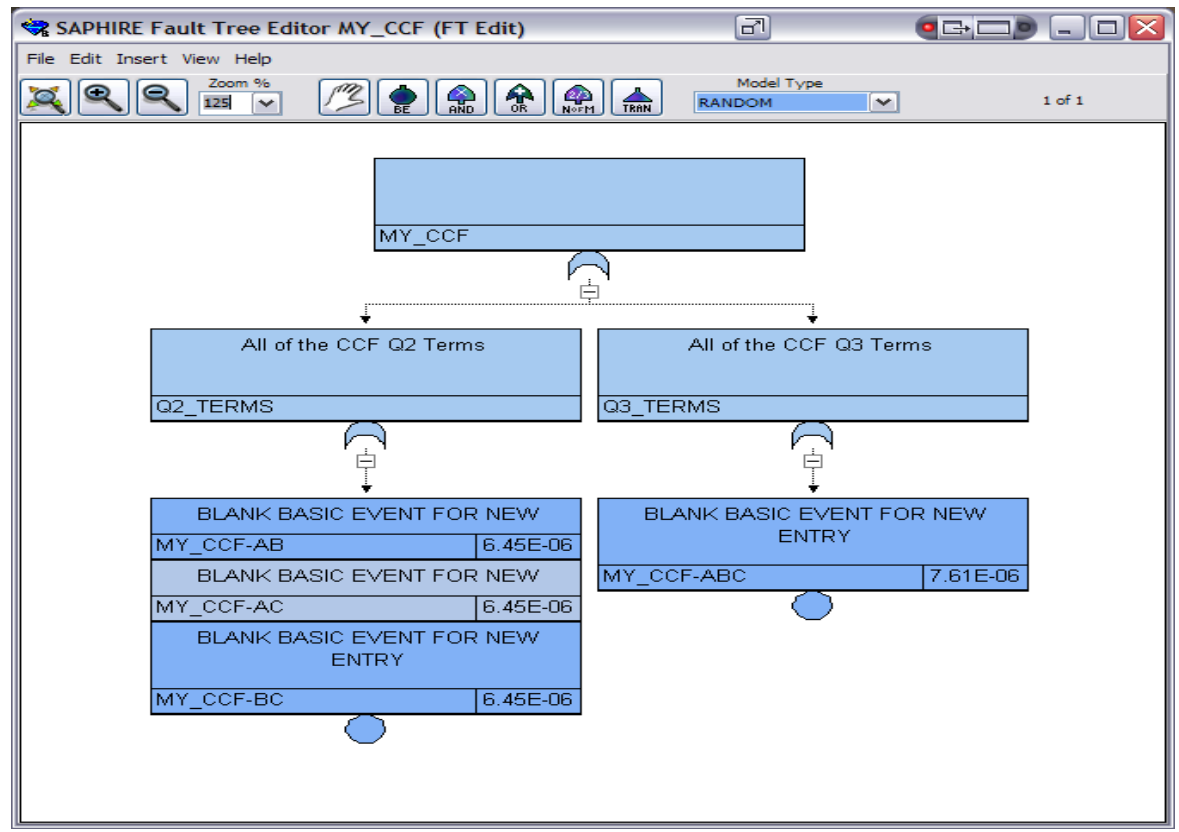

Figure 37. CCF Fault Tree 


\section{PHASE AND LERF MODELING}

SAPHIRE 8 has the capability to perform Phase and LERF modeling. Phases can be assigned to the project by opening the Project menu and selecting Phases as shown in Figure 38.

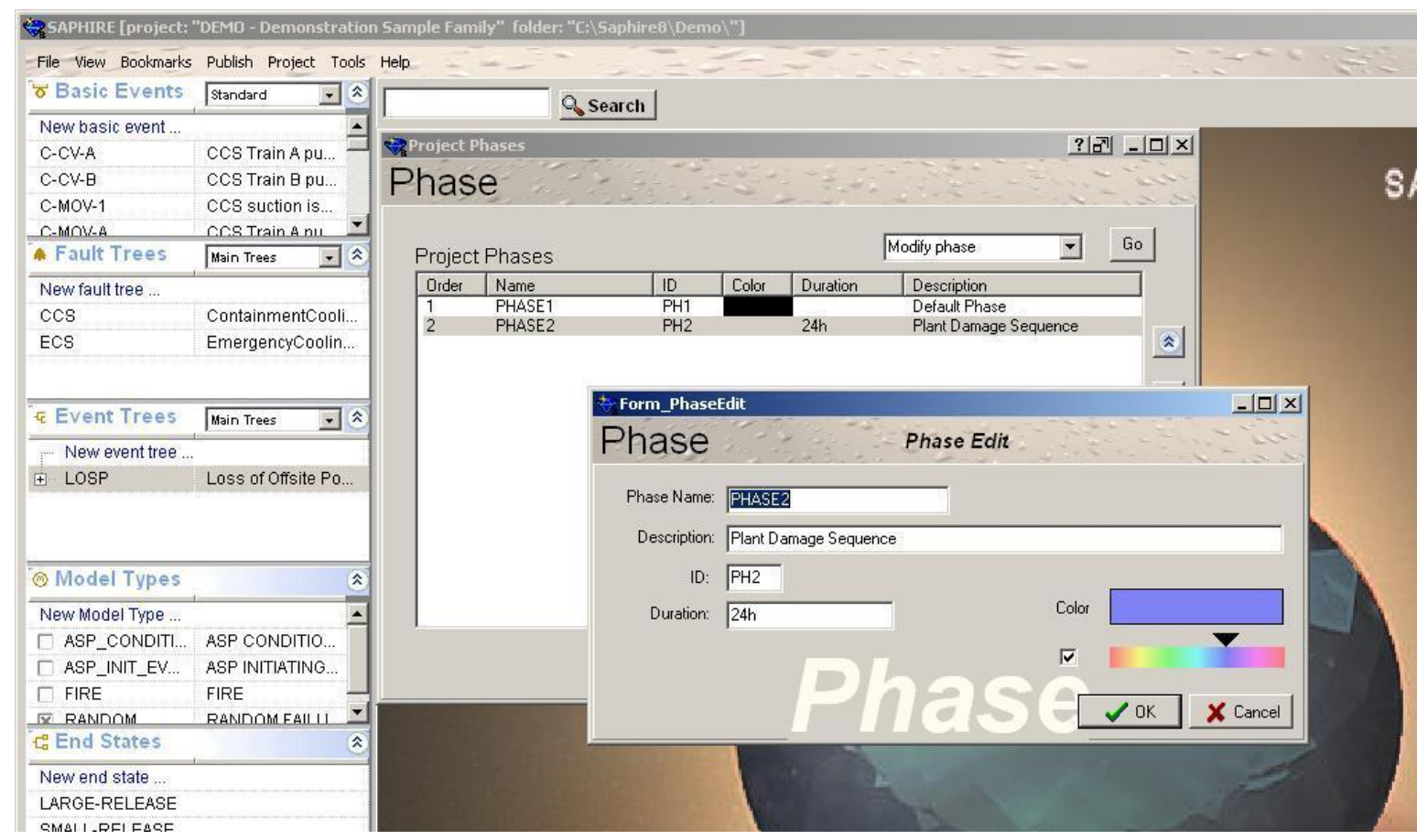

Figure 38. Adding a new Phase path

Phases can be assigned to objects in the Event Trees as shown in Figure 39 where End-States for the Level 1 phase lead to the PDS phase. Current functionality can only assign end-states based on phases. Transfers to the next levels of the model and analyses for such will be available in the near future. 


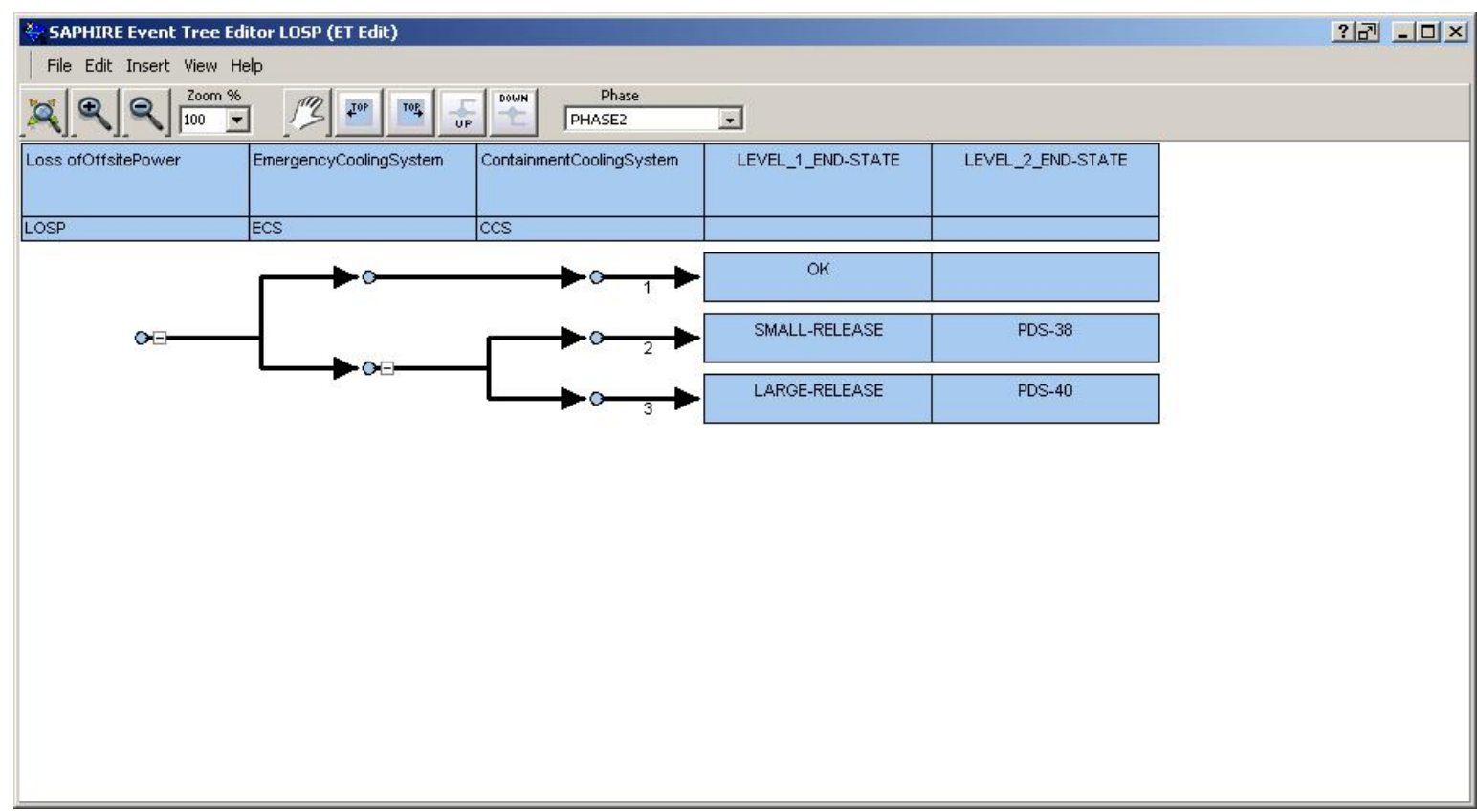

Figure 39. Example Event Tree with multiple level End-States

\section{External Events Modeling via an Accident Sequence Matrix}

The following describes the approach to which an "accident matrix" can be used to construct a portion of a SAPHIRE EE model. First, the accident matrix file should be created as an ASCII file using the following columns (separated by commas):

*Analysis Type, Initiating Event, IE Frequency, IE Description, Event Tree Name, Event Tree Description, X-Fer to, Flag Set Name, Flag Set Description, End State Substitution, Flagset Setting(s).

If any lines are meant to be comments, start them with a * or a $\mid$. Add each item desired as a new line and fill in the needed columns. An example is shown in Figure 40. 


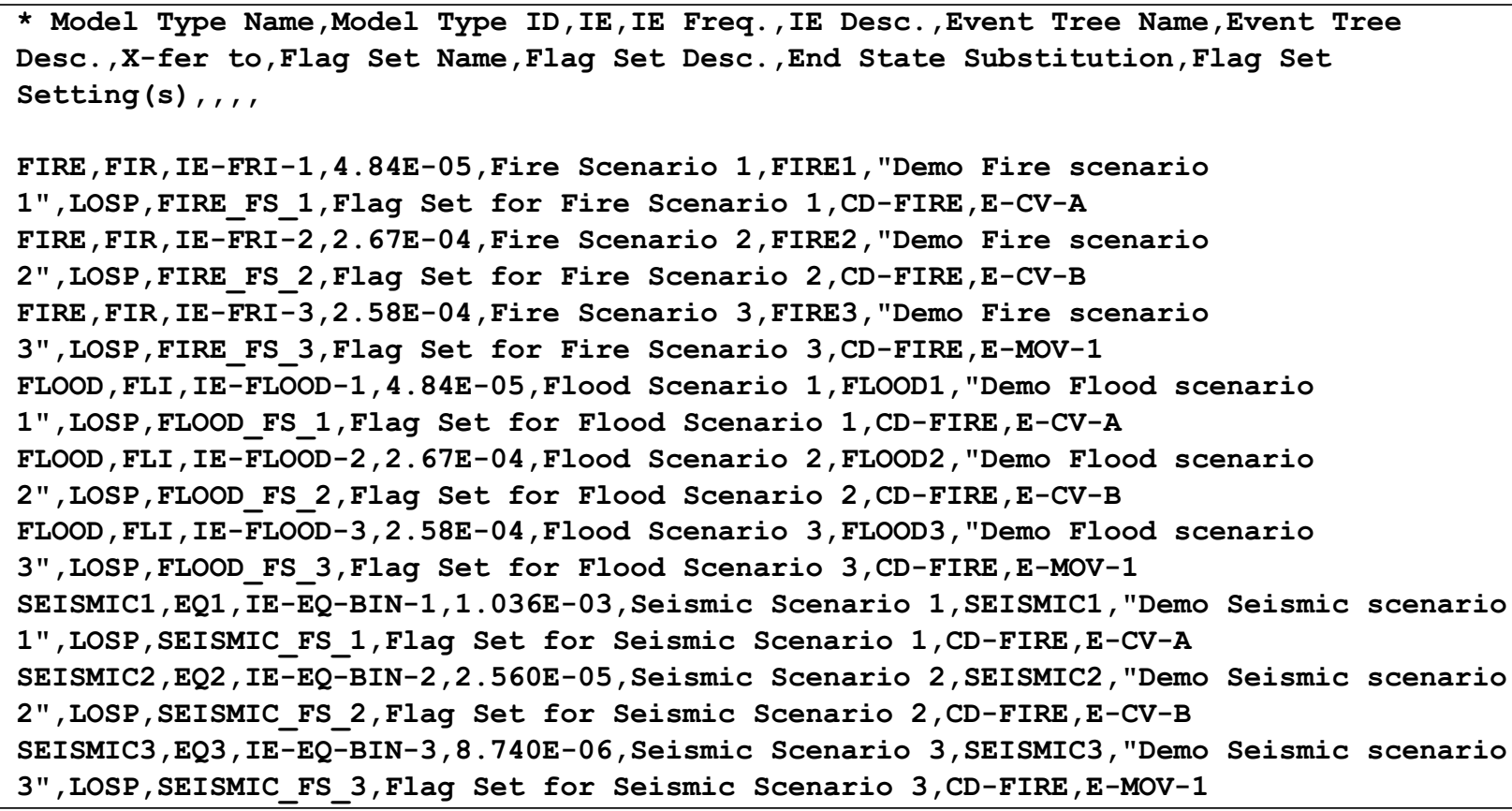

Figure 40. Information for an accident sequence matrix file.

In SAPHIRE, the accident matrix file is loaded via Project->Tools->Add Accident Matrix from the main menu. Click the open button and locate the accident matrix file desired. Click the Add button and the process begins. Information will appear in the text box (see Figure 41) as the accident matrix file is being processed. Any errors or warnings will also be displayed in this box. When done, click the OK button. The project will now be populated with the event trees and basic events described in the accident matrix file. 


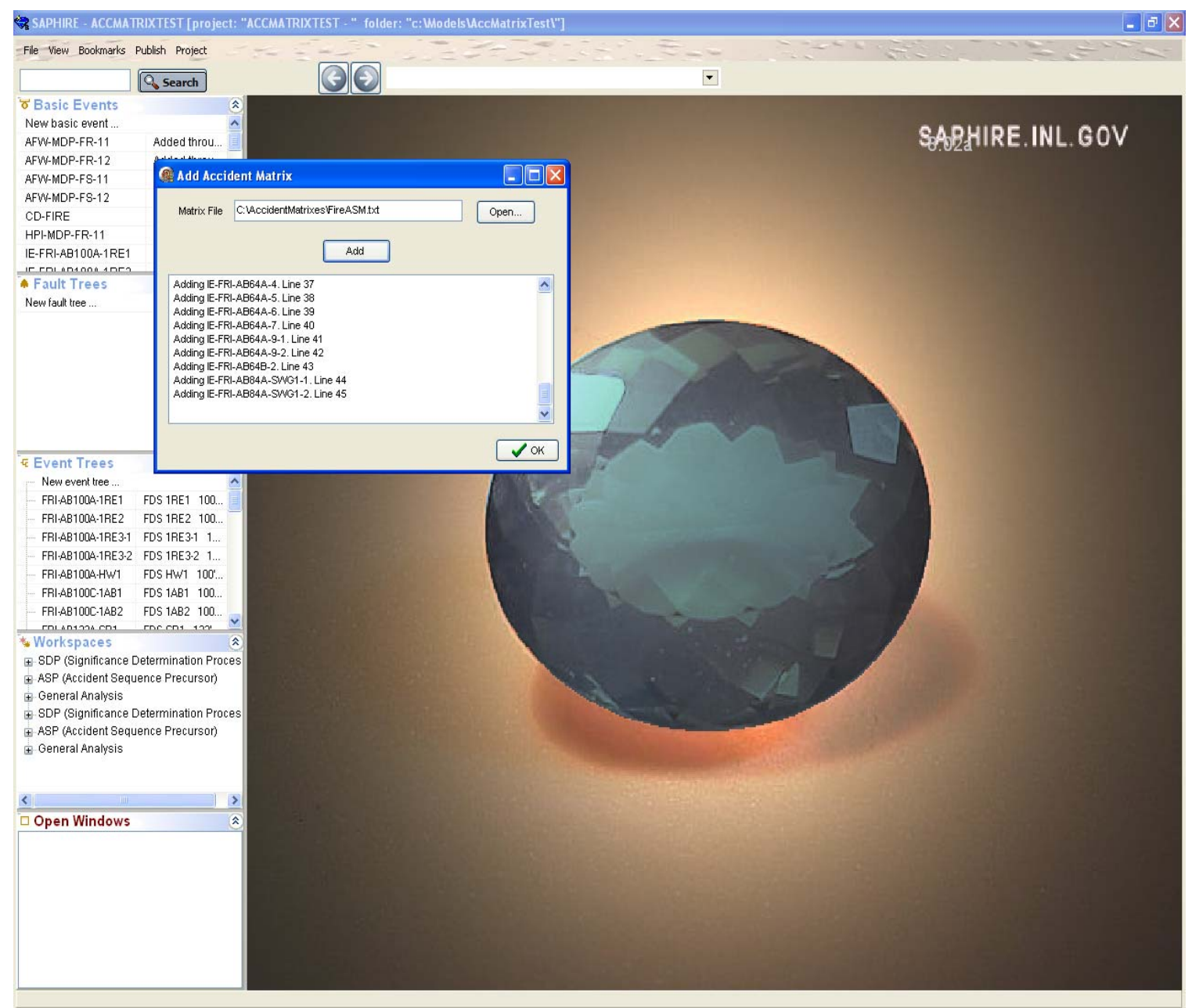

Figure 41. Processing the accident sequence matrix file.

\section{Loading and Extracting Information}

The Load and Extract option (under the File menu option) outputs MAR-D formatted files of the project information (when Extracting) or imports information (when Loading). The files to be loaded or extracted should be in the project subfolder labeled "Mard." To load MAR-D information, click the Load tab, then click the Open button to specify which MAR-D files to load. Once the files are specified, the applicable information will appear as checked in the object list (basic events, fault trees, event trees, etc.). Click Process to load the information. To Extract files, click the Extract tab (as shown in Figure 42). 


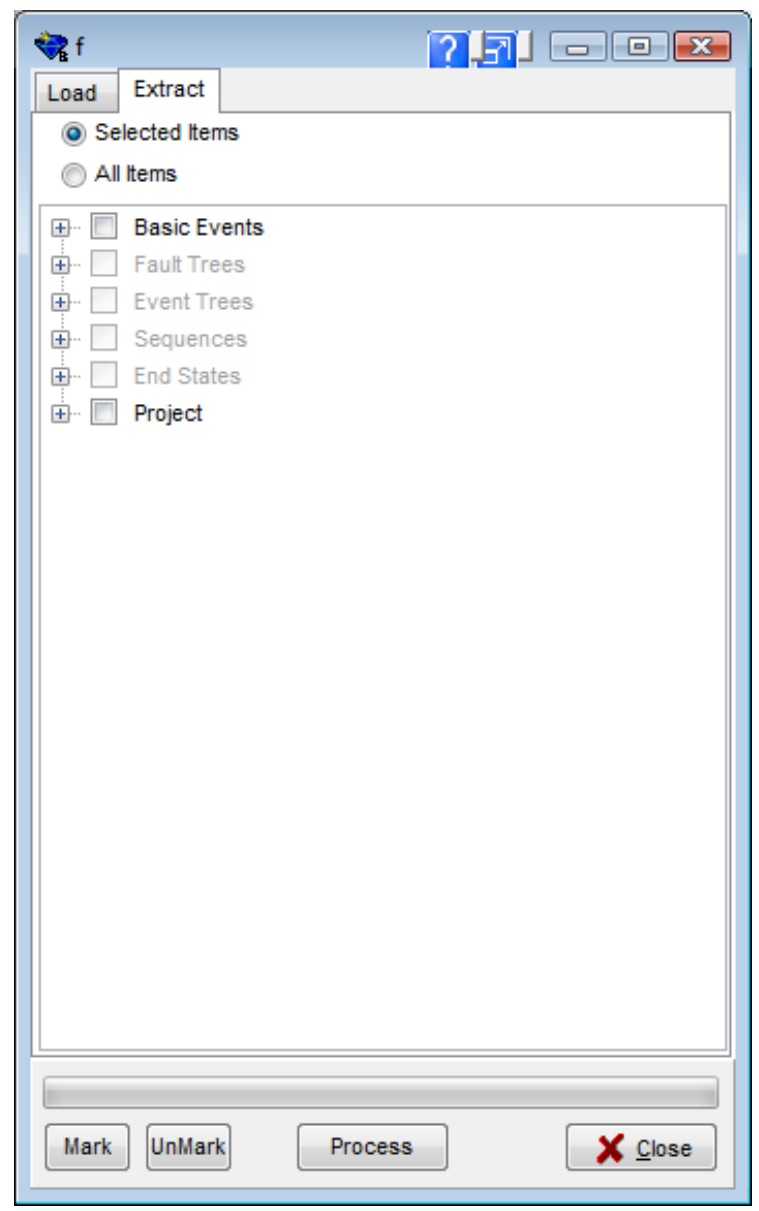

Figure 42. Load and extract option.

If items are already selected in the object lists in the main SAPHIRE window, one can extract MAR-D information for just these select items by clicking the Selected Items button. Alternatively, SAPHIRE will all output of all items within a particular object type by selecting the All Items button. The sub items for an object may be selected by using the check boxes under that object. To output the MAR-D file(s), click the Process button.

\section{WORKSPACES}

The workspace concept is introduced in SAPHIRE 8. The Workspaces menu is located in the left panel and contains analysis options available for the model type loaded (Figure 43). A model not identified as a SPAR model will only show the option for a General Analysis, which is a full option analysis. SPAR models also have the option for SDP and Events Assessment, which are tailored analyses for specific situations. 


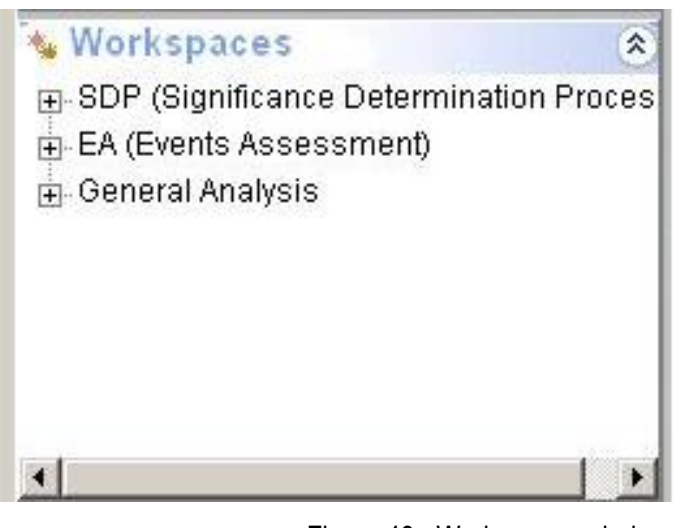

Figure 43. Workspaces window

Workspaces are created and saved separately from the base model which keeps the original database intact. Each workspace created can have multiple analyses performed and saved. SAPHIRE 8 saves the "current case" to the workspace and the immediately prior version of the model in the workspace is compared to (ala the "base case" in SAPHIRE 7). Each time the workspace calculation is performed, the changed model becomes the "base case" for the next calculation within that workspace. The logic in the original model is kept unchanged outside of the workspace. Workspaces are independent of each other and calculations made within one workspace will not affect another. Only changes made to the model in the base model area outside of the workspace environment will overwrite the original database.

\section{SOLVING PROJECT - Significance Determination Process (SDP)}

The Significance Determination Process (SDP) is a specific analysis function within SAPHIRE 8. This function is designed to analyze SPAR models to obtain results that represent the annualized change in core damage frequency. These models have all of their components and systems modified in order to be recognized by the SAPHIRE 8 modules that carry out this analysis. This module performs the Phase 2 SDP analysis using PRA models. The following will provide the steps and results of performing an SDP analysis.

The SDP analysis within SAPHIRE 8 is straight forward as far as setting up the model. The biggest challenge, like all analyses, is understanding the event and making sure it is being mapped correctly into the PRA model. SAPHIRE 8 is designed to walk the analyst through the required steps to perform an SDP analysis. There are four major steps in setting up the SDP analysis.

To start this process, the user selects SDP (Significance Determination Process) $\rightarrow$ New Analysis from the Workspaces menu option. 


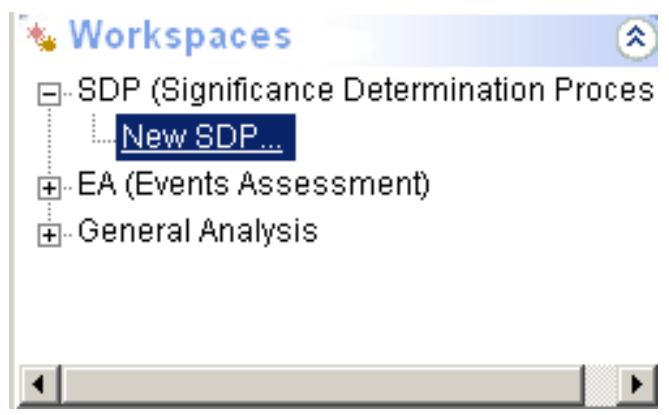

Figure 44. SDP Workspace creation path

This option requires a double click and then SAPHIRE copies all of the information from the project into a new workspace. By making this copy, any modifications will only be saved for this particular analysis and the original model will not be affected. SAPHIRE 8 then loads the step 1 analysis screen (Figure 45), which allows the user to start mapping the event into the PRA model.

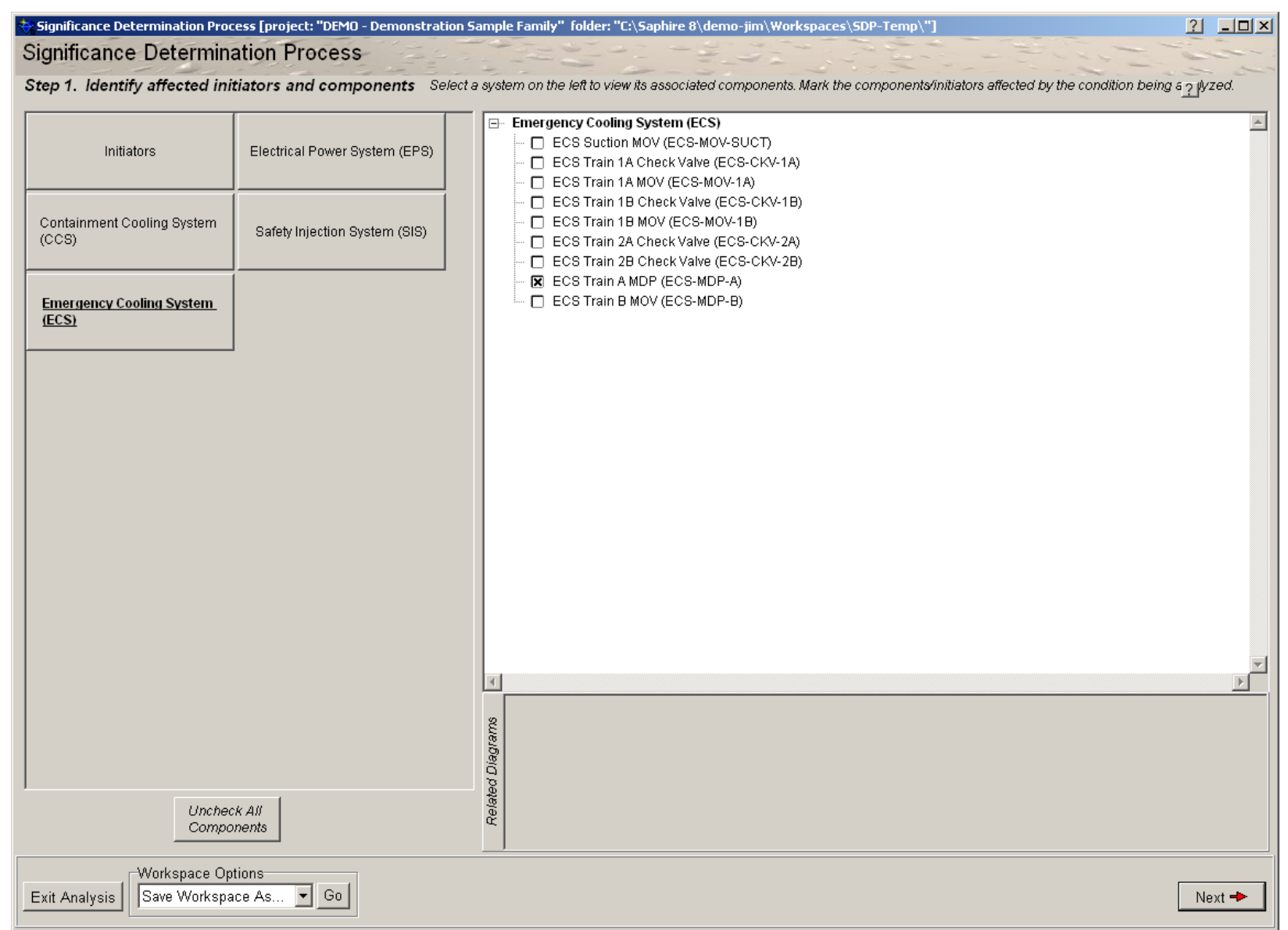

Figure 45. SDP Step 1 analysis screen

From the screen above, the analyst can select either the initiating event or component that needs to be adjusted based on the inspection finding. To perform this step, select the appropriate system and SAPHIRE will then list all components that are modeled under that system. The actual components need 
to be checked from the list shown in the right hand box. By checking the component, SAPHIRE will know this component will be modified for this particular analysis. Once all components have been checked, select the Next button to move to Step 2.

The screen shown above has a button called "Uncheck All Components", which will remove all checked components in order to start a new analysis. Also, the there is an option that will allow the analyst to save the workspace, by selecting the Go button next to the "Save Workspace As..." text. This save option will store all of the mapped components and modifications to a separate workspace that will remain with the project.

After the Next button is selected, SAPHIRE will show all of the components that were selected. This is step 2 in the SDP process, which allows for the components to be modified. This screen (Figure 46) provides the analyst with 2 options for modifying the component in question. The two options are:

1. New Failure Probability

2. True (Component is Failed).

The first option allows the analyst an opportunity to either increase/decrease the components probability based on the information from the inspection. The second option states the component did fail. This option will automatically adjust the common cause failure probability event. The CCF event is modified, because the modified failure failed not only that particular event, but also has the potential of failing all similar components due to the same failure mechanism.

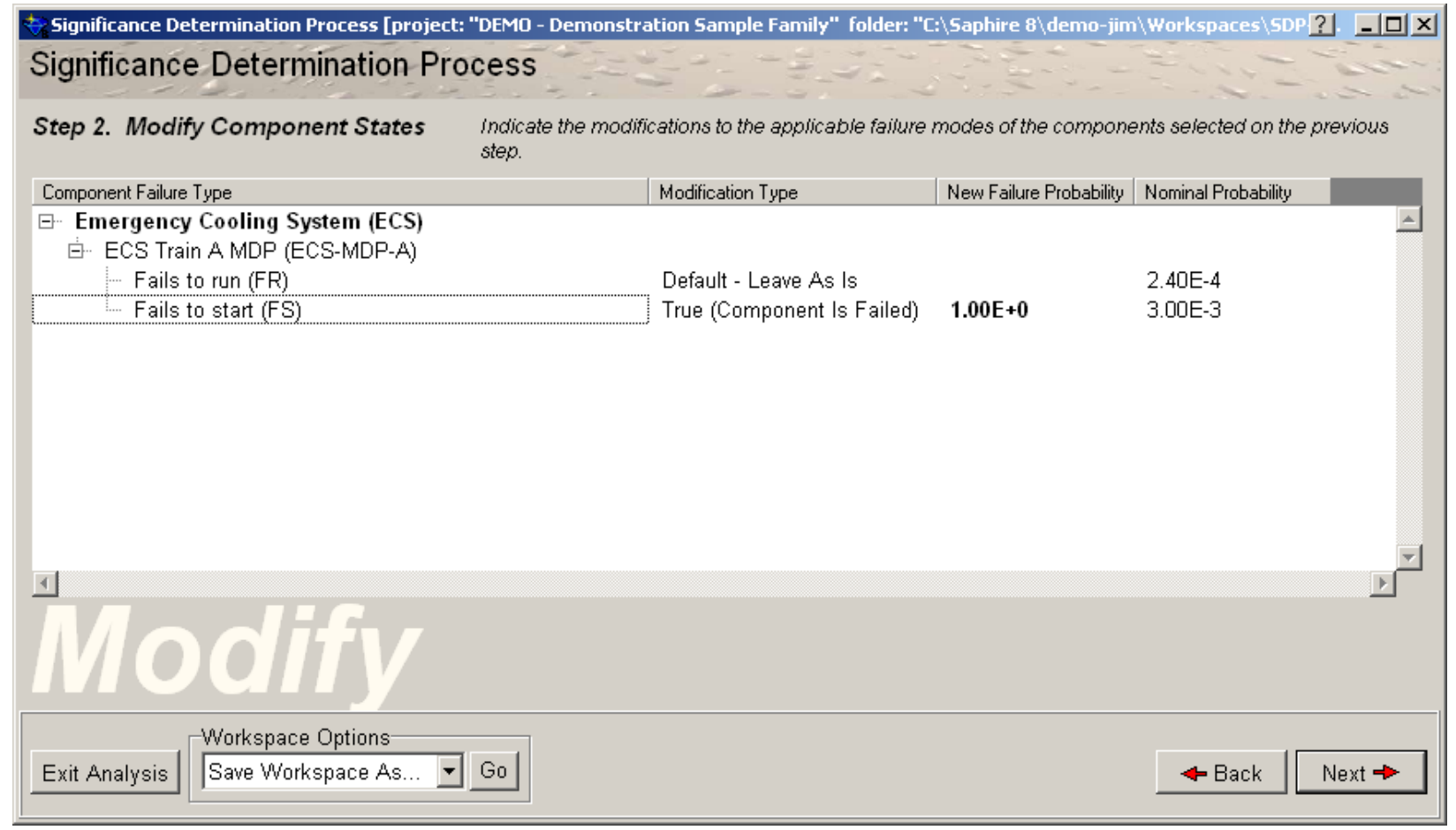

Figure 46. SDP Step 2 analysis screen 
Once all of the components have been modified, the Next button is selected. SAPHIRE will now move to the third step (Figure 47). Step 3 is the analysis options (called Analyze Changes). This option is used to set up the duration of the event that was found in the inspection. The starting date and time for the analysis defaults to the time listed from the computer. This date and time can be changed to the actual start time of the event. The next part is the duration or when the event was discovered and corrected. There are two ways of making this modification. The first way is to click the End Date radio button and directly input the date and time. The second way is to specify duration in either hours, days, weeks, or months.

The next options found on this screen are the addition of a title and descriptive information. SAPHIRE provides a place for the title of the analysis and a notebook page to include as much information as possible about the event. This information is carried along and stored with the analysis. The final results will report this information.

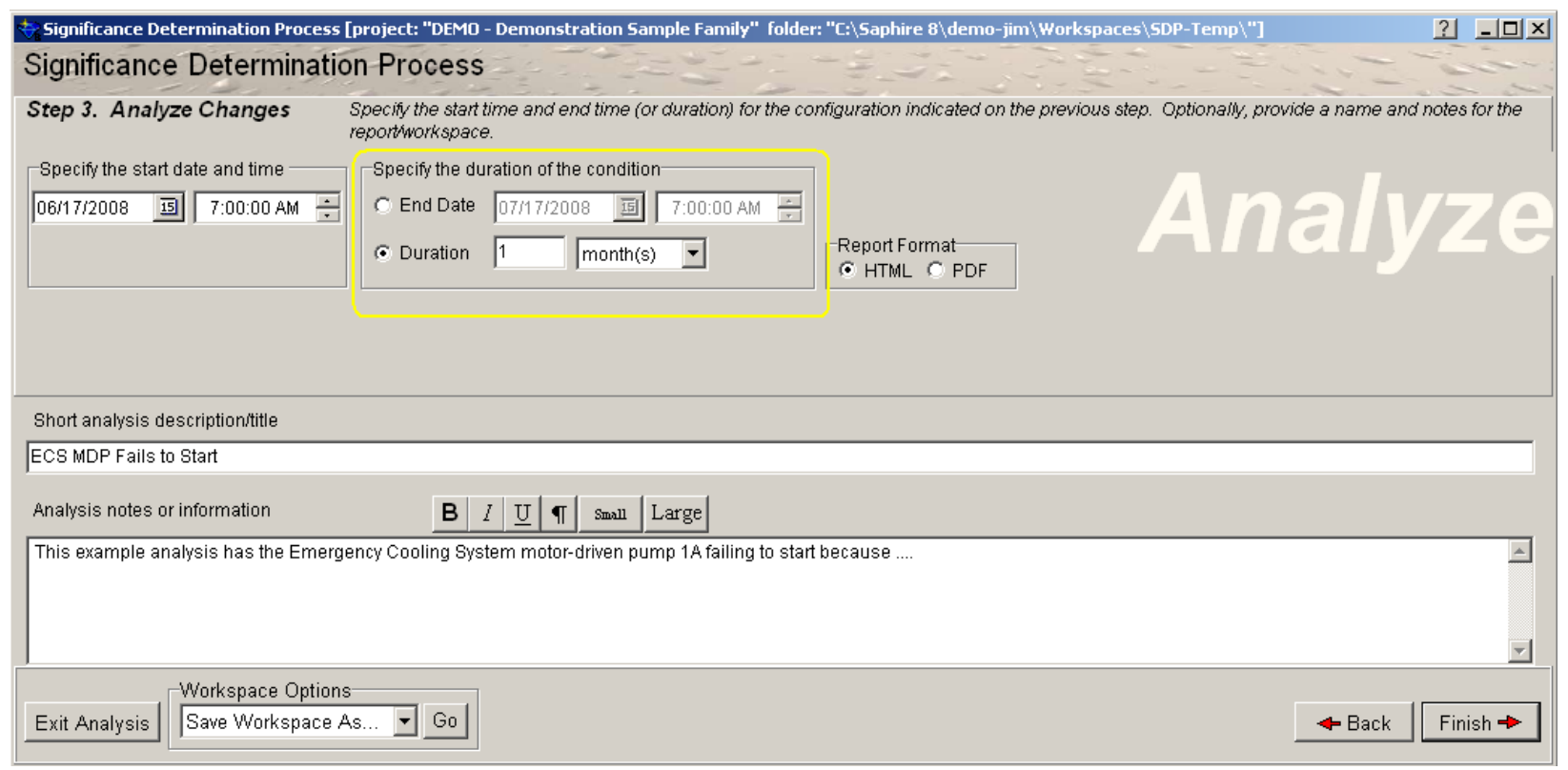

Figure 47. SDP Step 3 analysis screen

Once the duration has been input along with the analysis title and description, the Finish button needs to be clicked. This will start the analysis option. A SAPHIRE pop up screen will appear letting the analyst know that the analysis can take some time to complete. Click "OK" to allow SAPHIRE to continue with the analysis.

? The SDP process needs to perform/update the analysis by performing a calculation. Depending on the model and the specified changes, this calculation could take some time to complete.

Press OK to continue the calculation, or Cancel to stop.

$$
\text { OK Cancel }
$$


SAPHIRE will provide a complete overview of the analysis in the form of five different sections. The distinct sections are: I. Summary, II. Insights, III. Figures, IV. Notes, and V. SPAR Model Issues. The overall report is shown below.

I. Summary: The first section provides a summary that includes a graph (Figure 49) illustrating the annualized CDF and its risk significance, the duration of the condition, and the component(s) that were unavailable along with its failure setting.

II. Insights: The second section provides information about the results. The output listed in this section is based on the final risk significance. The higher the risk significance, the more information is provided.

III. Figures: The third section provides a variety of graphs representing the evaluation.

Figure III-A - The first figure provides a risk profile of the event (Figure 50).

Figure III-B - The second figure provides a pie chart showing the percent contribution to the annualized CDF given the condition (Figure 51).

Figure III-C - The third figure shows the relative risk change graphed by the initiating events given the condition (Figure 52).

Figure III-D - The fourth figure shows the change in annualized CDF as function of duration (Figure 53).

IV. Notes: The fourth section provides the output of the notes the analyst typed into the analysis. By reporting the notes, all of the information is documented and kept together.

V. SPAR Model Issues: Each SPAR model comes with a disclaimer discussing implications of using the model. Included in the disclaimer are points of contacts related to the models and the SDP software.

Increase in Yearly Core Damage Frequency

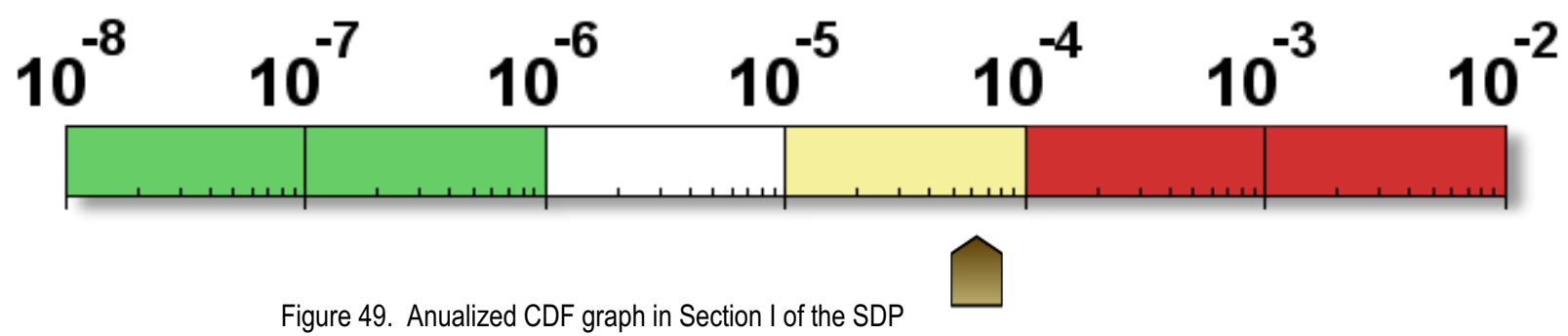

I. Summary: Condition: Yellow: 5.9E-05/yr

The given condition duration is 1 week.

Emergency Cooling System (ECS) ECS Train A MDP (ECS-MDP-A) had adjustments made to the following failure modes: 
- Fails to start (FS) was changed from 3.00E-3 to True (Component Is Failed). This implies that the component was failed for the entire duration.

Multi-pass option with cut set update calculation used (Preferred method).

Used truncation value of $1 \mathrm{E}-12$

Model Version: 0.0 Model Date: --/--/----

\section{Insights:}

The following accident sequences were most impacted (by total increase):

\begin{tabular}{|l|c|l|c|c|}
\hline Event Tree & Sequence & Failed Systems & Increase & $\begin{array}{c}\text { (Factor } \\
\text { Increase) }\end{array}$ \\
\hline LOSP & 2 & ECS & $5.8 \mathrm{E}-5$ & $(2.3 \mathrm{E}+2)$ \\
\hline LOSP & 3 & ECS, CCS & $9.1 \mathrm{E}-8$ & $(1.1 \mathrm{E}+0)$ \\
\hline
\end{tabular}

The following components contribute the most to the annualized CDF:

\begin{tabular}{|l|r|}
\hline \multicolumn{1}{|c|}{ Components } & \multicolumn{1}{c|}{ Percent Contribution } \\
\hline RWST failure & $56.0 \%$ \\
\hline Diesel Generator B Fails to start & $26.0 \%$ \\
\hline Diesel Generator B Fails to run & $16.0 \%$ \\
\hline ECS Train B MDP Fails to start & $3.9 \%$ \\
\hline Diesel Generator A Fails to run & $0.5 \%$ \\
\hline
\end{tabular}

\section{Figures}




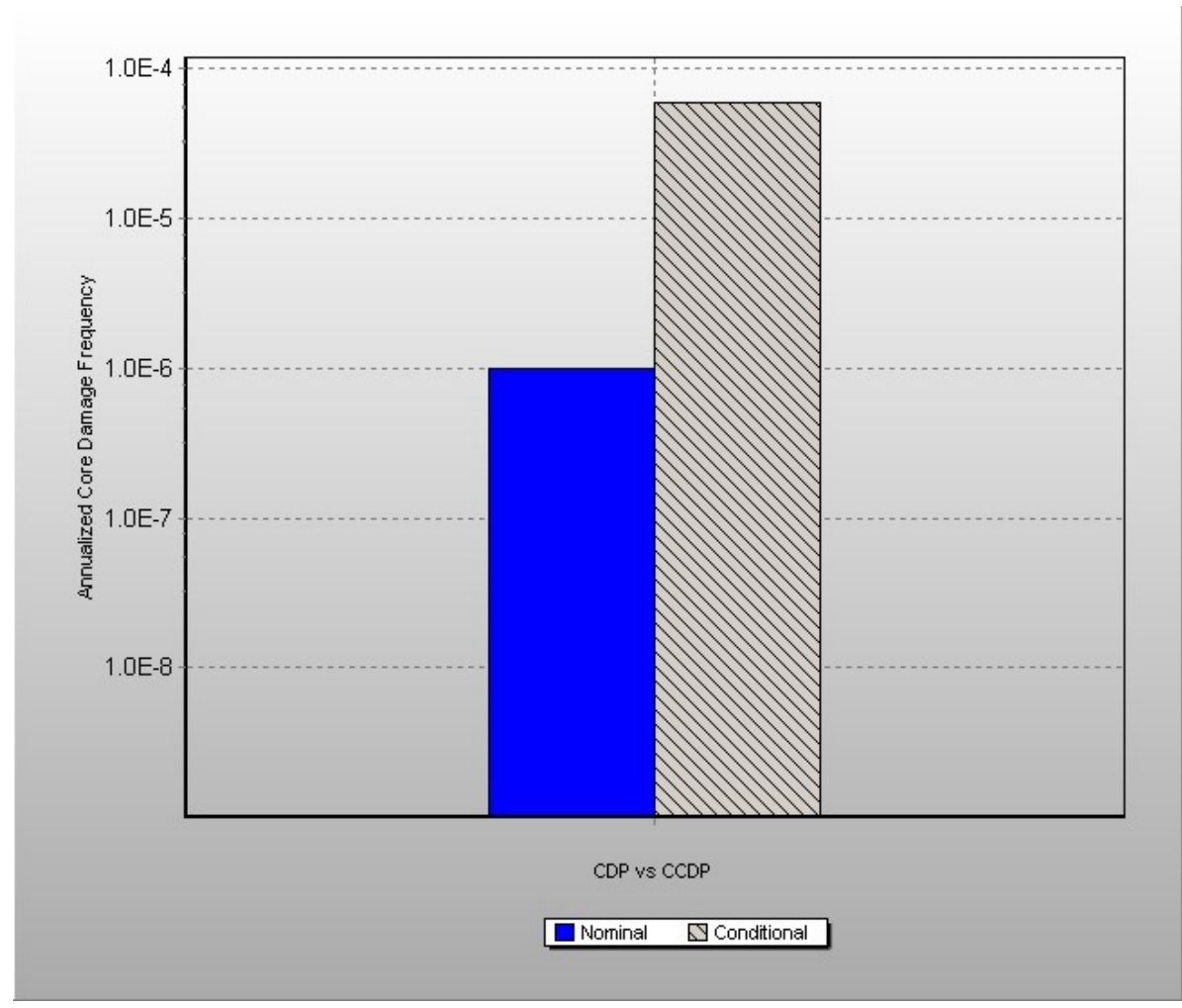

Figure 50. Relative risk change for the condition (Figure III-A of SDP report) 


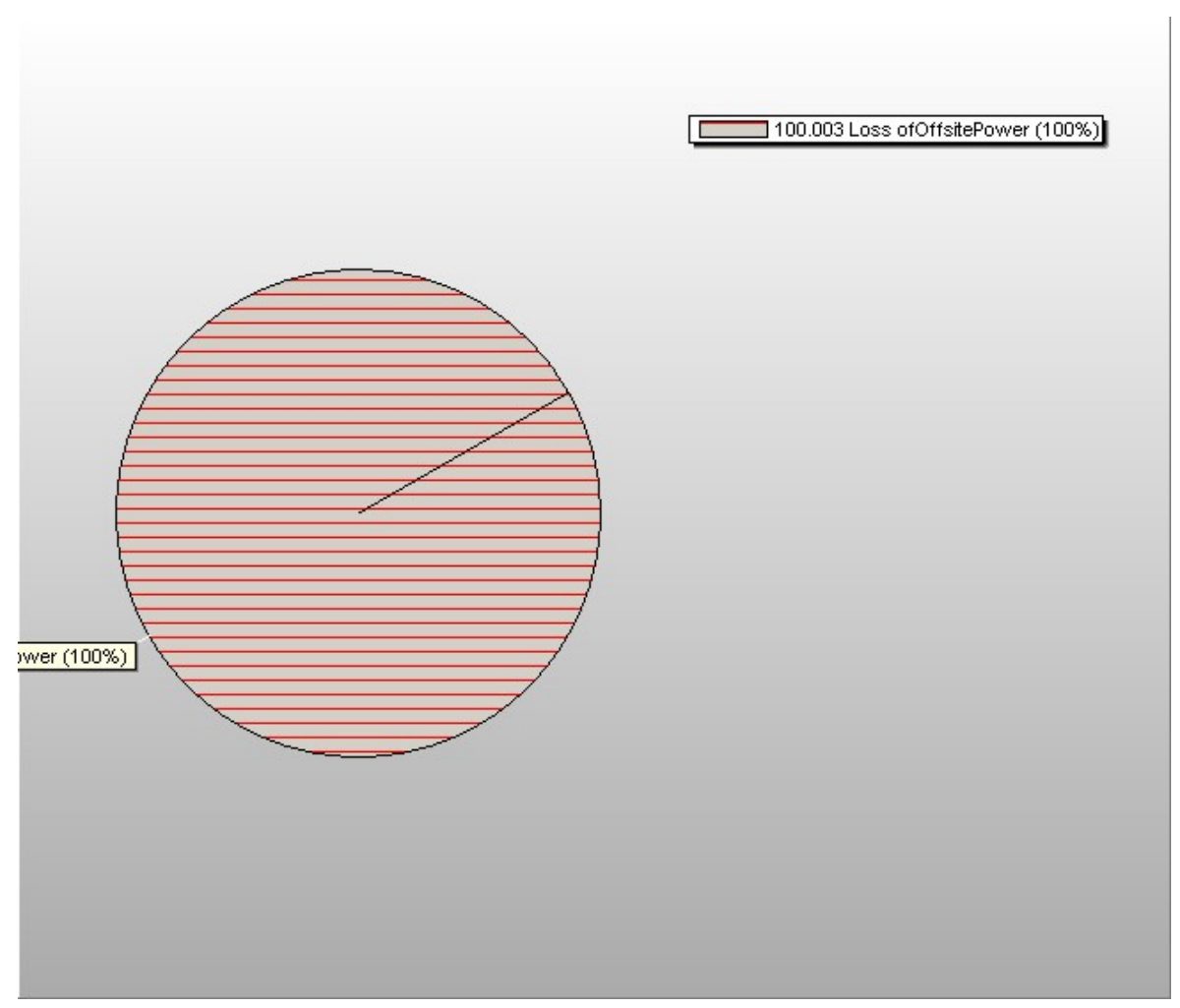

Figure 51. Percent contribution of initiating events to the increase in CDF (Figure III-B of SDP report)

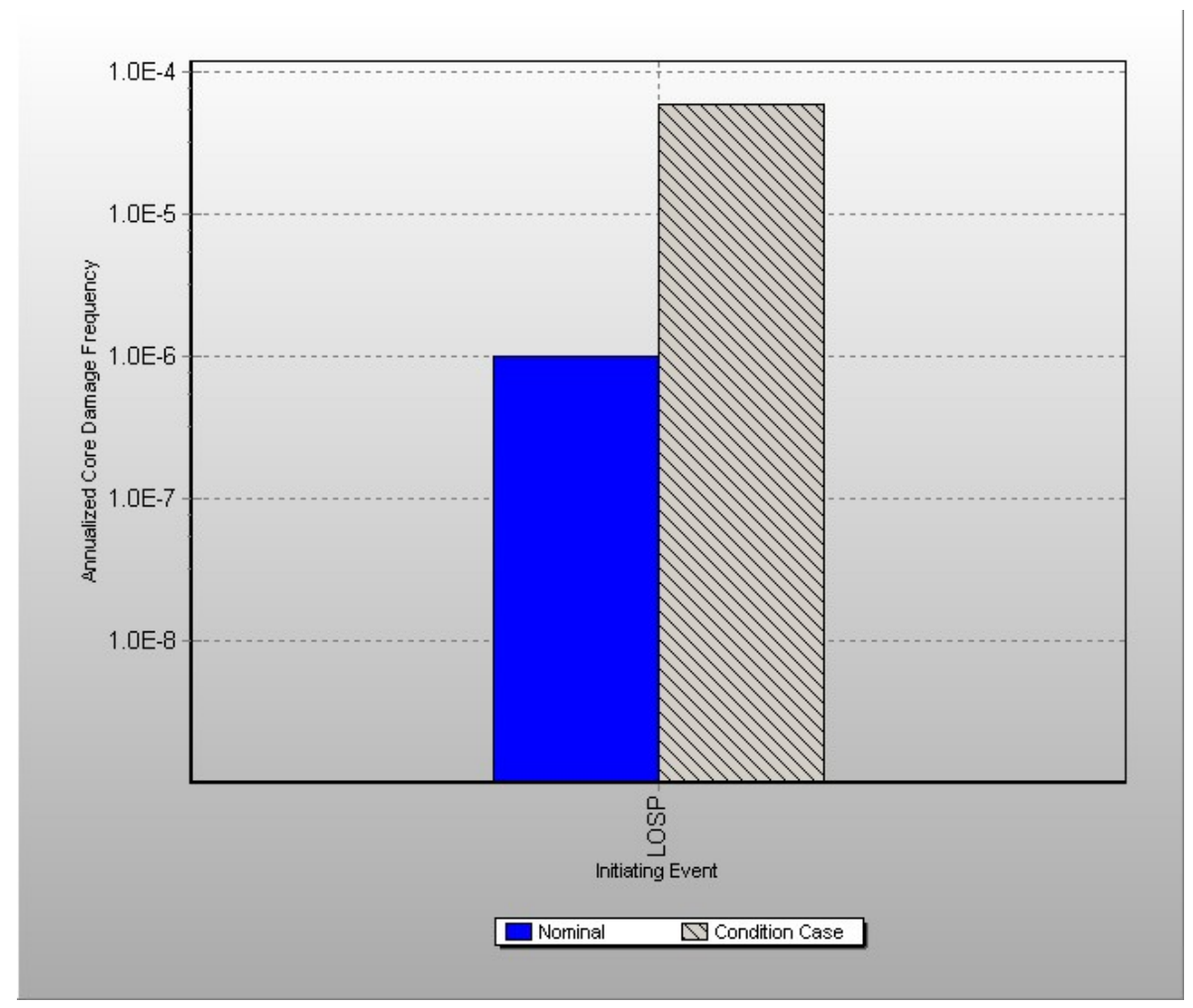

Figure 52. Relative risk change grouped by initiating event (Figure III-C of SDP report) 


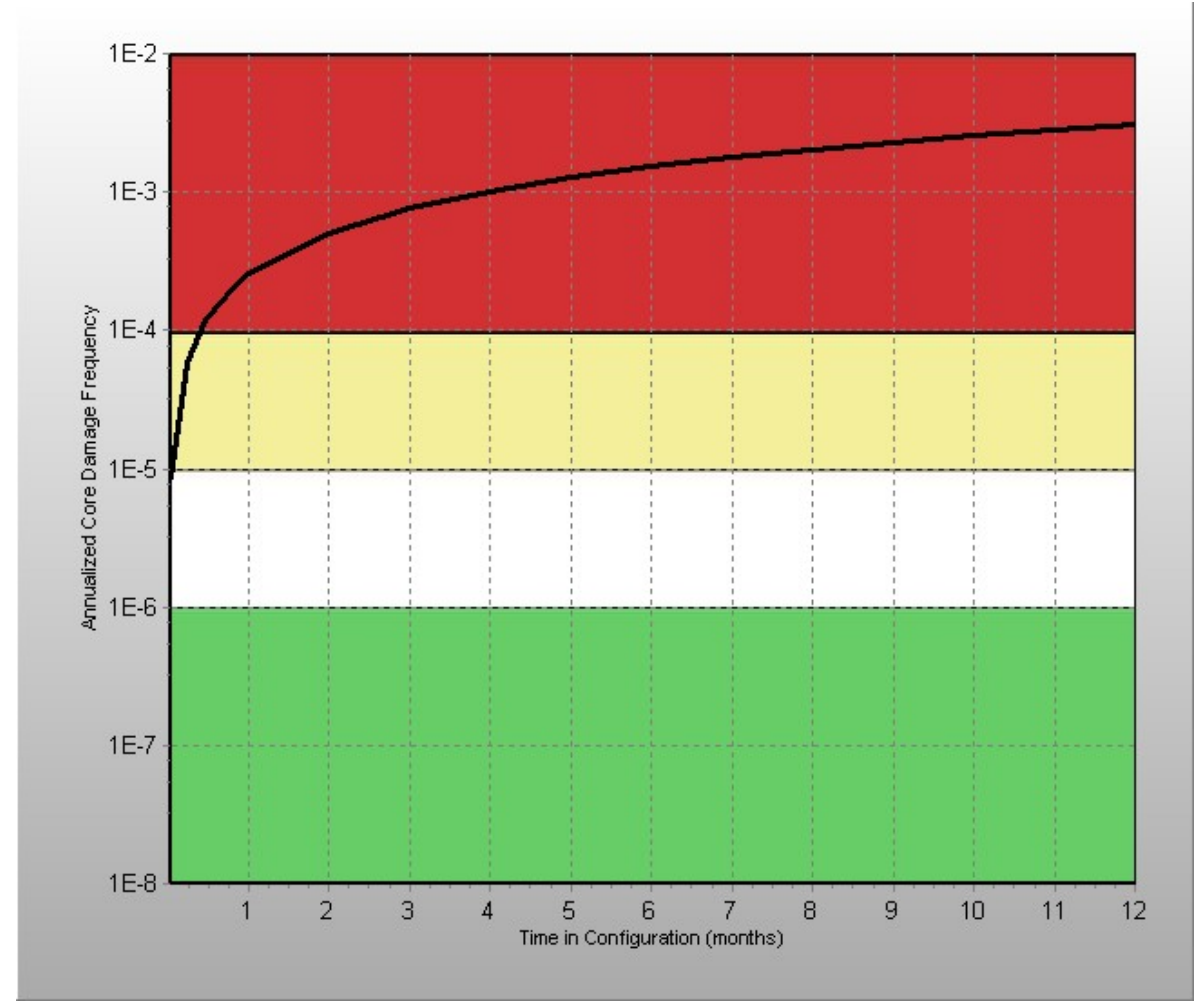

Figure 53. Change in annualized CDF as a function of duration (Figure III-D of SDP report)

IV. Notes: This example analysis has the Emergency Cooling System motor-driven pump $1 \mathrm{~A}$ failing to start because ....

V. SPAR Model Issues: Although the Revision 3 SPAR models are distributed for unrestricted use, nevertheless, analysts are advised to understand the inputs and assumptions inherent in the models and to apply model results accordingly. The Revision 3 SPAR models have been designed to capture the significant risk contributors associated with internal event initiators for plants operating at power. Therefore, these models may not provide the level of detail necessary to capture the risk from less significant contributors. Thus, for applications that relate to these contributions, the analyst may be required to make the appropriate modifications to the models.

\section{SOLVING PROJECT - Event Assessment (EA)}

The event assessment (EA) process performs two types of analyses on the PRA model. The first type is an initiating event assessment. This type of assessment is designed to analyze the PRA model given the initiating event occurred. The results are a condition core damage probability, since the PRA model is solved with the specific initiating event set to 1.0. The other assessment type is a conditional assessment. This type of assessment is based on a component(s) being failed or potentially unavailable to perform its safety function for some duration. The results from this analysis are also a conditional core damage 
probability. Each of these assessment types will be discussed below along with the SAPHIRE screen shots.

\section{Initiating Event Assessment}

To start the event assessment process, the analyst selects the EA (Events Assessment) option from the Workspaces menu option.

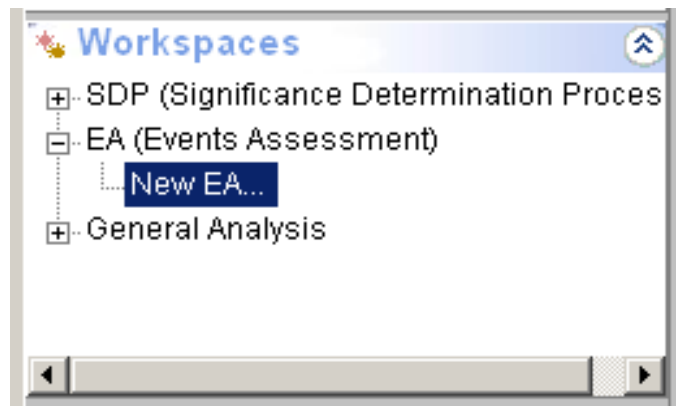

Figure 54. Event Assessment workspace path

This option requires a double click and then SAPHIRE copies all of the information from the project into a new workspace. By making this copy, any modifications will only be saved for this particular analysis and the original model will not be affected. SAPHIRE 8 then loads the Event Assessment Type analysis screen, which allows the analyst to select the type of assessment by selecting the appropriate radio button to start mapping the event into the PRA model.

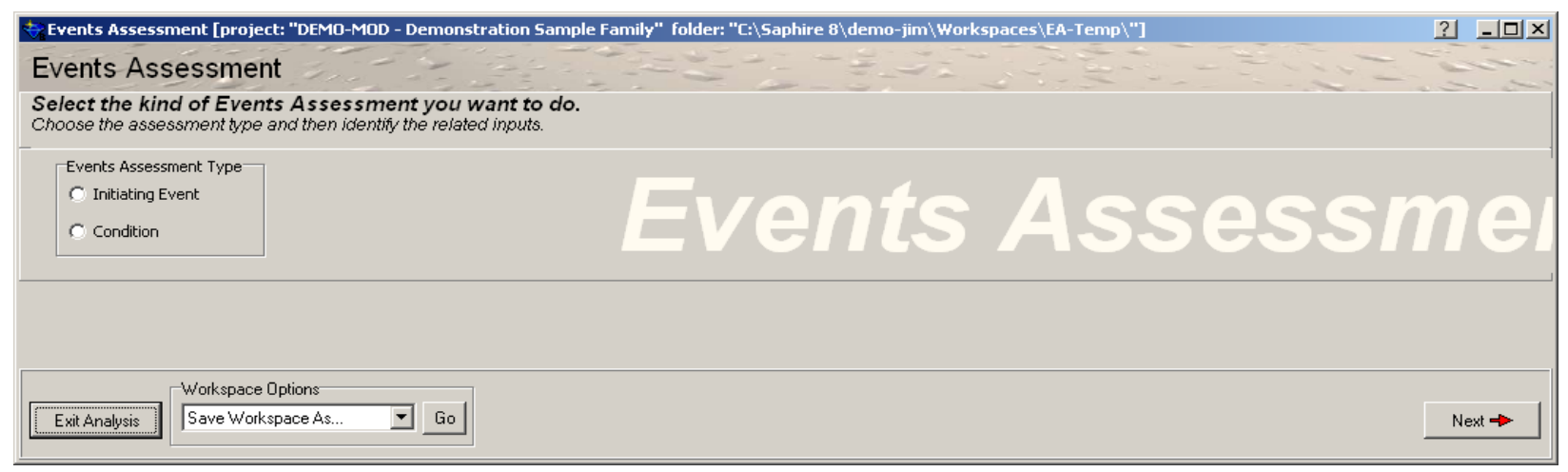

Figure 55. Events Assessment Type analysis screen

Once the Initiating Event assessment type radio button is selected, SAPHIRE loads up all of the initiating events developed in the PRA model (Figure 56). The analyst then selects the initiating event that occurred and selects the Next button. 


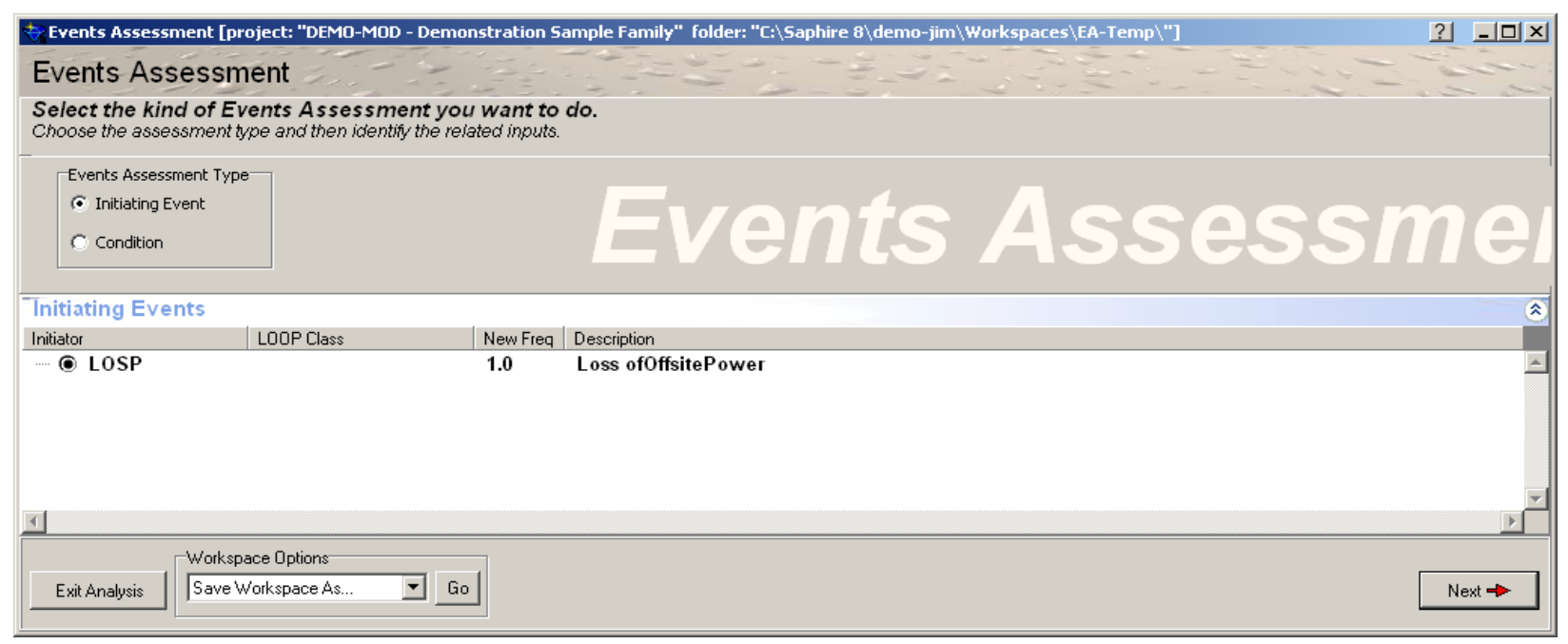

Figure 56. EA Initiating Events selection screen

SAPHIRE now displays a screen that contains all of the basic events found in the project (Figure 57). This list is used to finalize the assessment by selecting those component(s) that failed or could potentially fail if required during the mission time. After all of the affected components have been selected, the Next button is clicked to continue the assessment process.

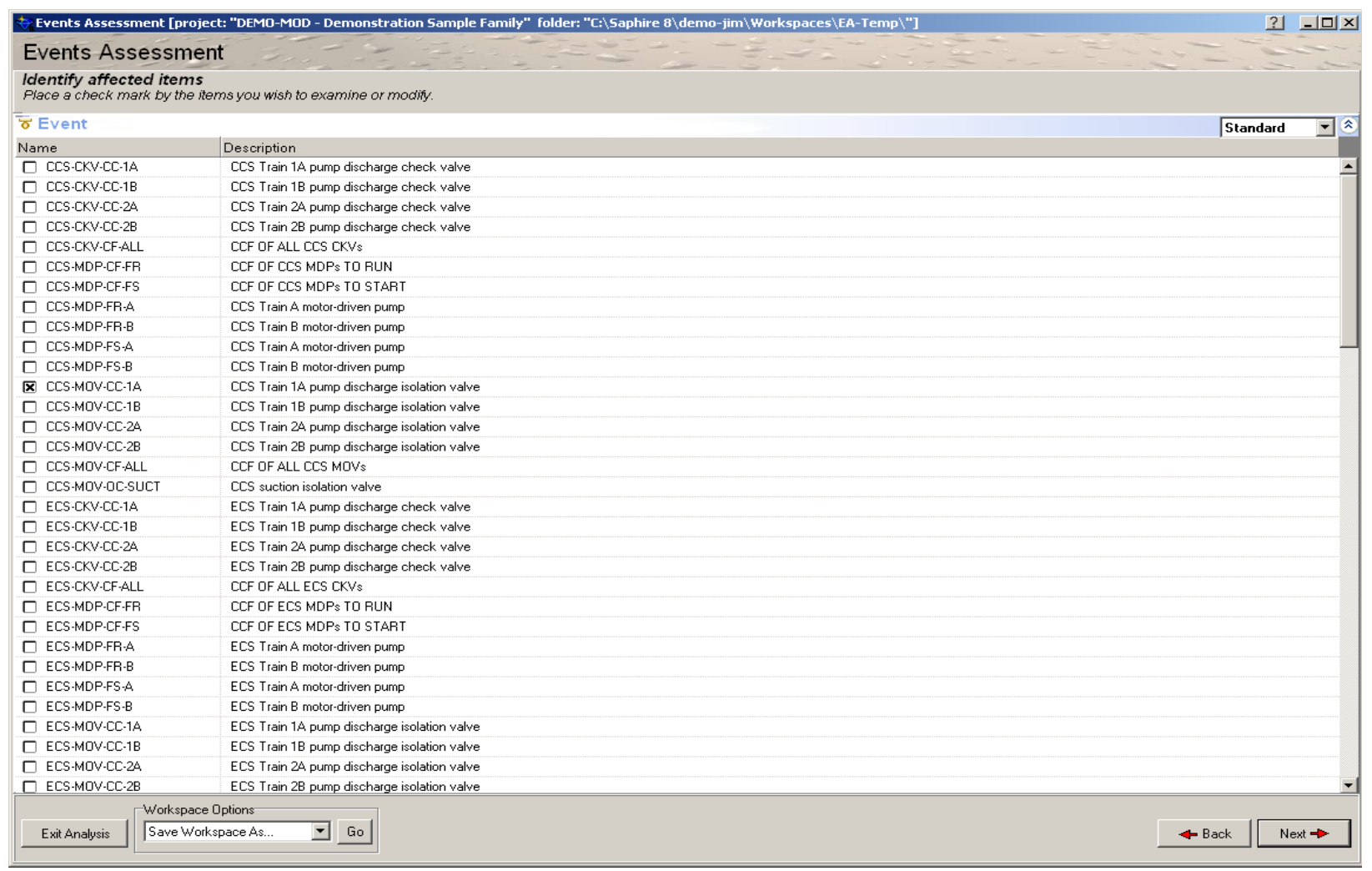

Figure 57. EA Basic Events selection screen 
The next screen is designed to make the probability modifications to the selected component(s) (Figure 58). There are four probability options that can be selected for the component(s).

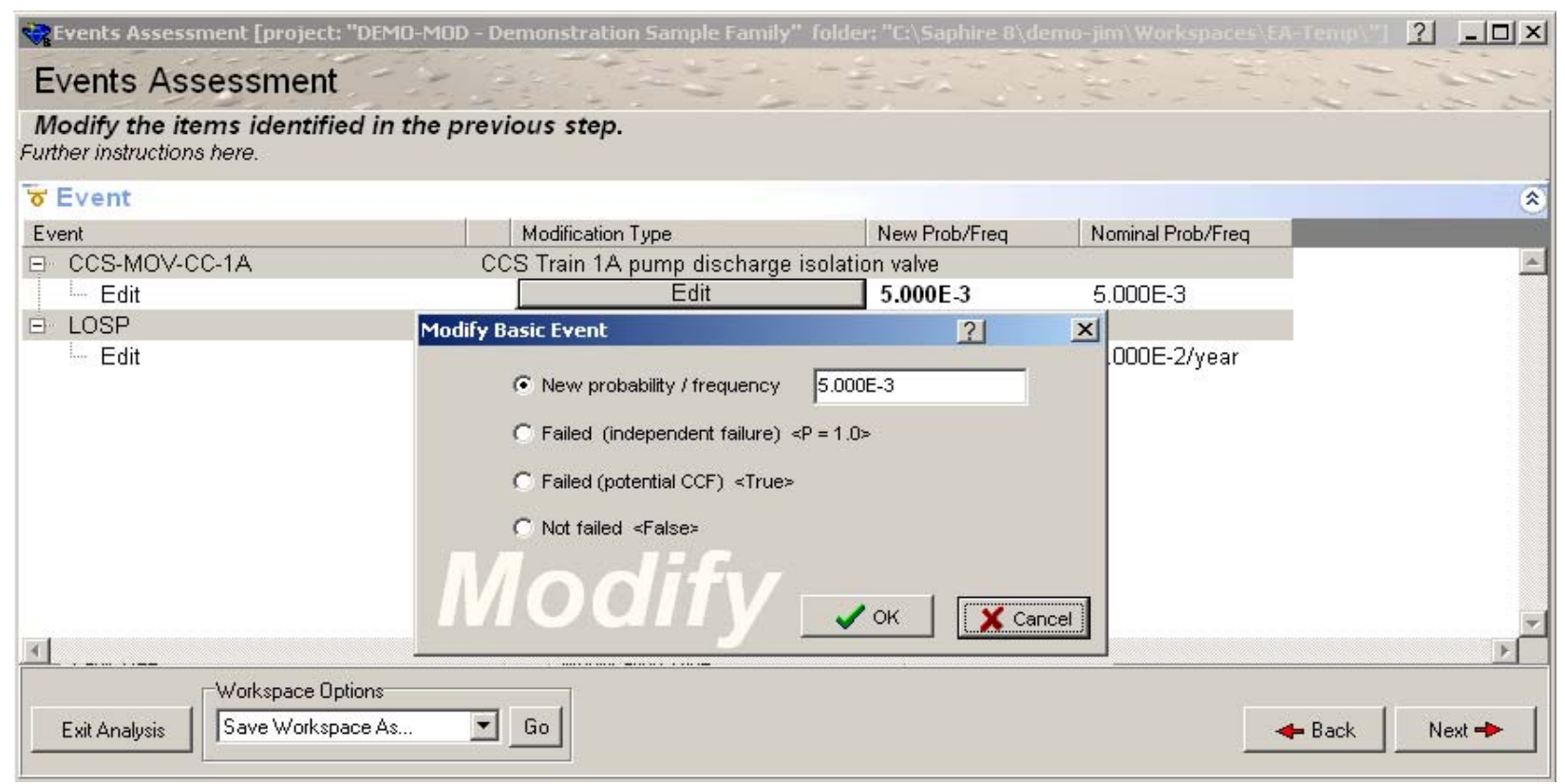

Figure 58. EA Basic Event modification screen

The first option allows for the component's probability to be changed from its nominal probability to some other value. This value could be any value between 0.0 and 1.0. The next option tells SAPHIRE that the component failed but its failure was an independent failure. This means that the mechanism that caused the component to fail will not cause any similar components to fail the same way (i.e., no common mode failure). By selecting this radio button, SAPHIRE will not change the component's common cause failure probability, since it is assumed that the failure was an independent failure. The next radio button sets the component to a True house event, which tells SAPHIRE that the component(s) failed due to a potential common cause shock; therefore, SAPHIRE will automatically adjust the component's common cause failure probability. The last radio button tells SAPHIRE that the component can not fail, house event False.

Once the component's probabilities have been modified, the Next button is selected. The next screen provides the analyst with solve options (Figure 59). The analyst can select whether a single or multiple pass solution is required. Again, single pass will only solve the model and apply the recovery rules. The multiple pass will solve the model, apply the recovery rules, and perform a cut set update to remove any potential non-minimal cut sets that could have been created from the recovery rules. The specification of the truncation level to be used is specified along with the uncertainty information (number of samples, seed number, and type). Lastly, a title for the analysis along with a description of the event can be input into their appropriate spots. This will keep all of the information together. 


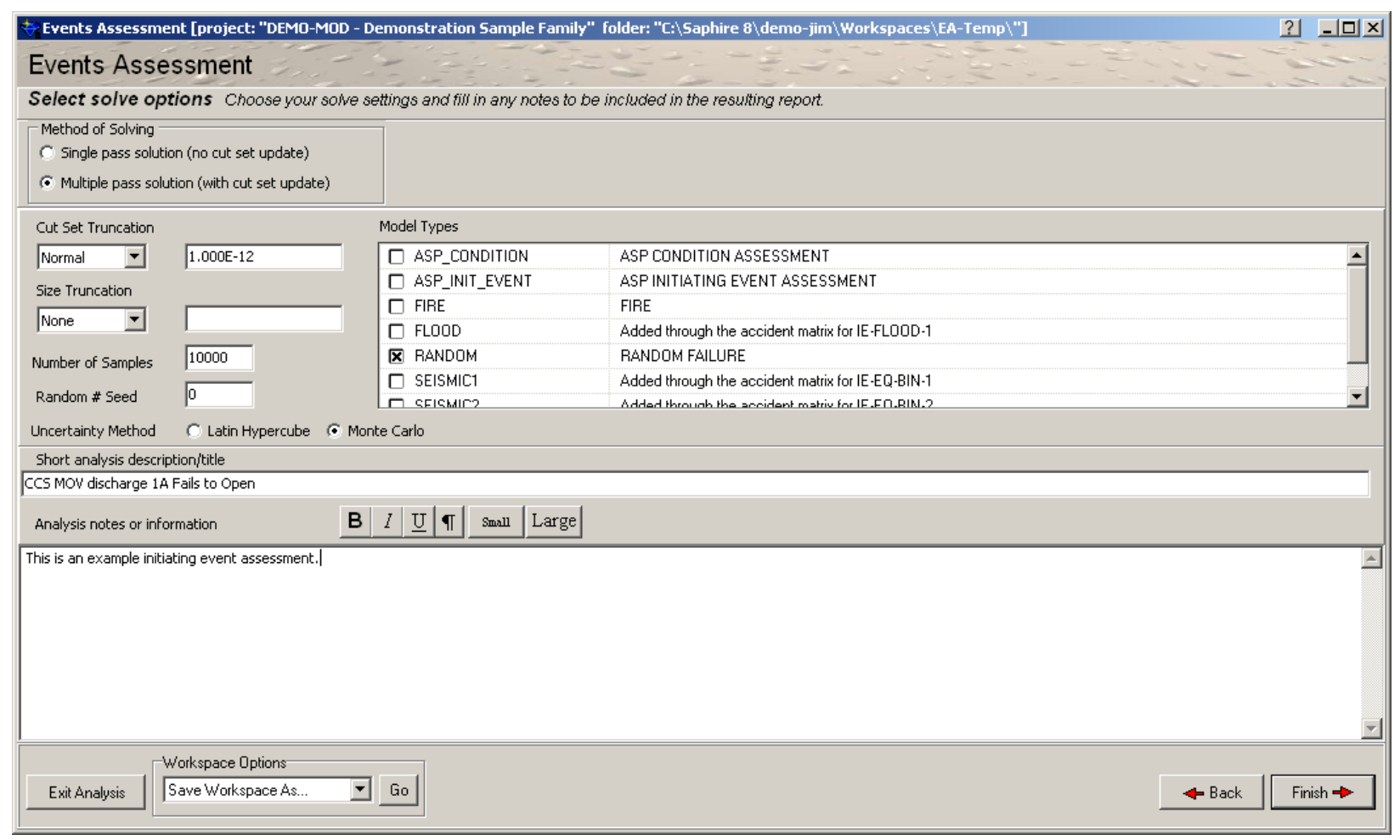

Figure 59. EA Solve options screen for Initiating Event assessment

Now that all of the information has been specified, click the Finish button to analyze the event. SAPHIRE will provide an output of the assessment on the last screen (Figure 60). 


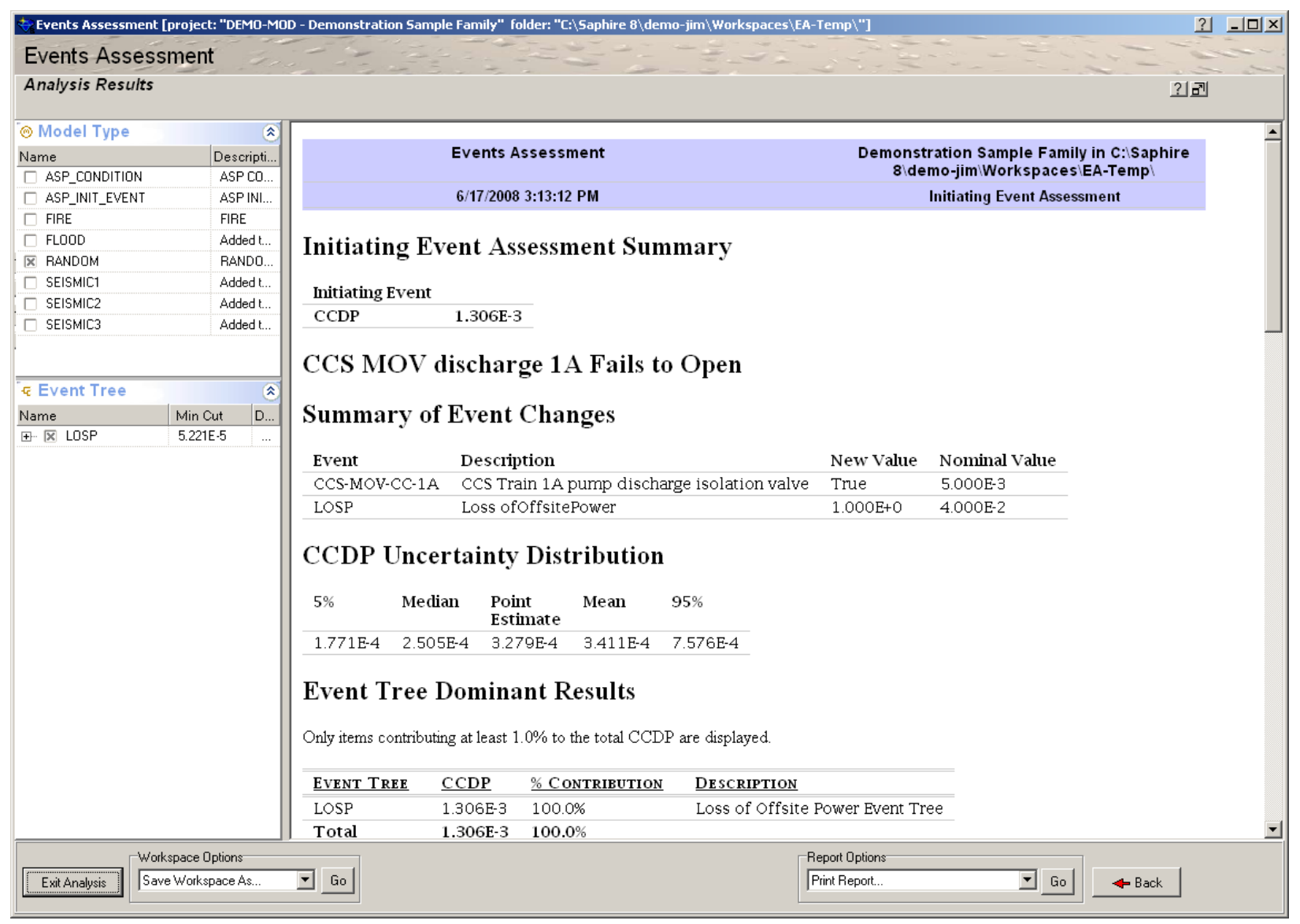

Figure 60. EA results screen for Initiating Event assessment

\section{Condition Assessment}

To start the event assessment process, the analyst selects the EA (Events Assessment) option from the Workspaces menu option (Figure 54).

This option requires a double click and then SAPHIRE copies all of the information from the project into a new workspace. By making this copy, any modifications will only be saved for this particular analysis and the original model will not be affected. SAPHIRE 8 then loads the Event Assessment Type analysis screen (Figure 55), which allows the analyst to select the condition assessment by selecting its radio button to start mapping the event into the PRA model.

Once the condition assessment type radio button is selected, SAPHIRE loads up the condition duration screen (Figure 61). This screen is used in the final calculation of the conditional core damage probability. (For condition assessments, the results are Importance $\mathrm{EA}_{\mathrm{A}}=\mathrm{CCDF}^{*}$ duration $-\mathrm{CDF}^{*}$ duration, where the CCDF is the results of the mapped event in the PRA and the CDF is nominal frequency of the PRA.) The analyst then specifies the duration of the event in hours, days, weeks, months or years, and selects the Next button. 


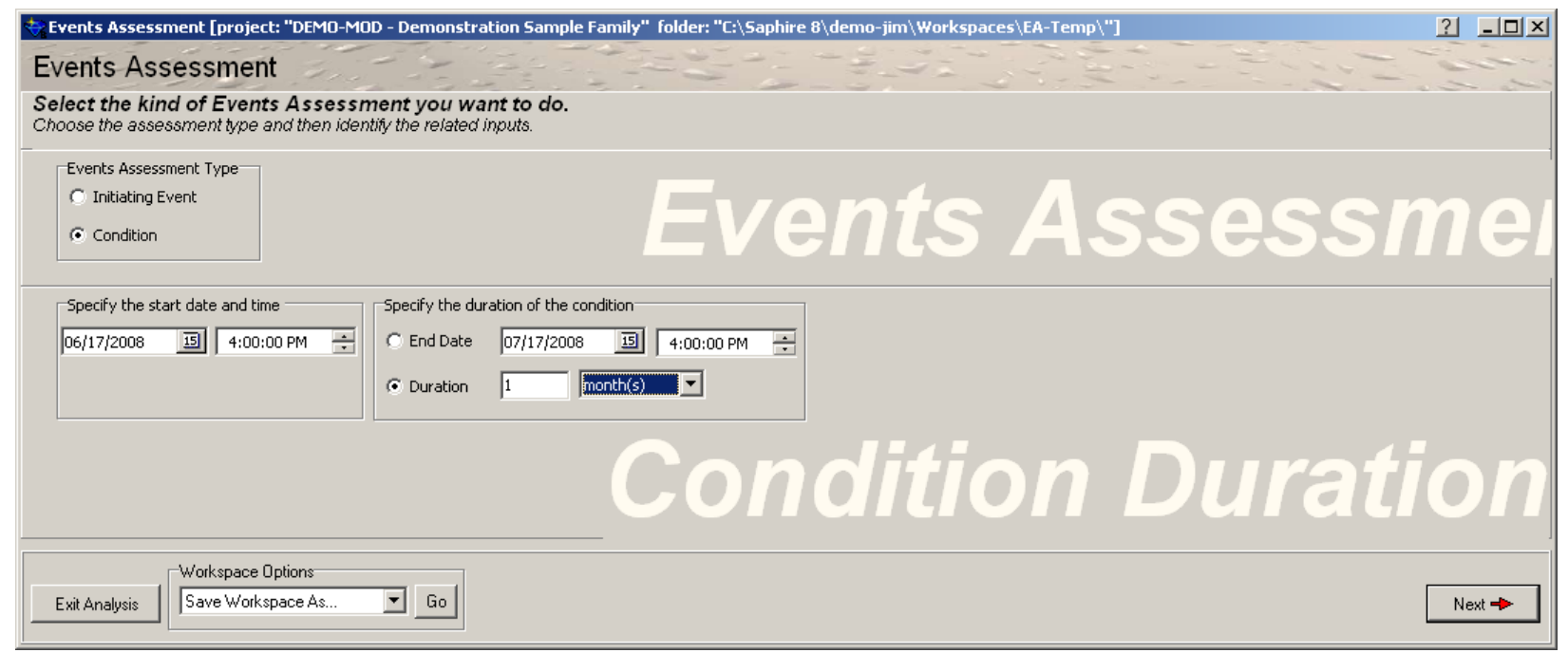

Figure 61. EA condition duration screen

SAPHIRE now displays a screen that contains all of the basic events found in the project (Figure 57). This list is used to finalize the assessment by selecting those component(s) that failed or could potentially fail if required during the mission time. After all of the affected components have been selected, the Next button is clicked to continue the assessment process.

The next screen is designed to make the probability modifications to the selected component(s). There are four probability options that can be selected for the component(s). These have been discussed in the initiating event assessment section (Figure 58).

Once the component's probabilities have been modified, the Next button is selected. The next screen provides the analyst with solve options (Figure 62). The analyst can select whether a single or multiple pass solution is required. Again, single pass will only solve the model and apply the recovery rules. The multiple pass will solve the model, apply the recovery rules, and perform a cut set update to remove any potential non-minimal cut sets that could have been created from the recovery rules. The specification of the truncation level to be used is specified along with the uncertainty information (number of samples, seed number, and type). Lastly, a title for the analysis along with a description of the event can be input into their appropriate spots. This will keep all of the information together. 


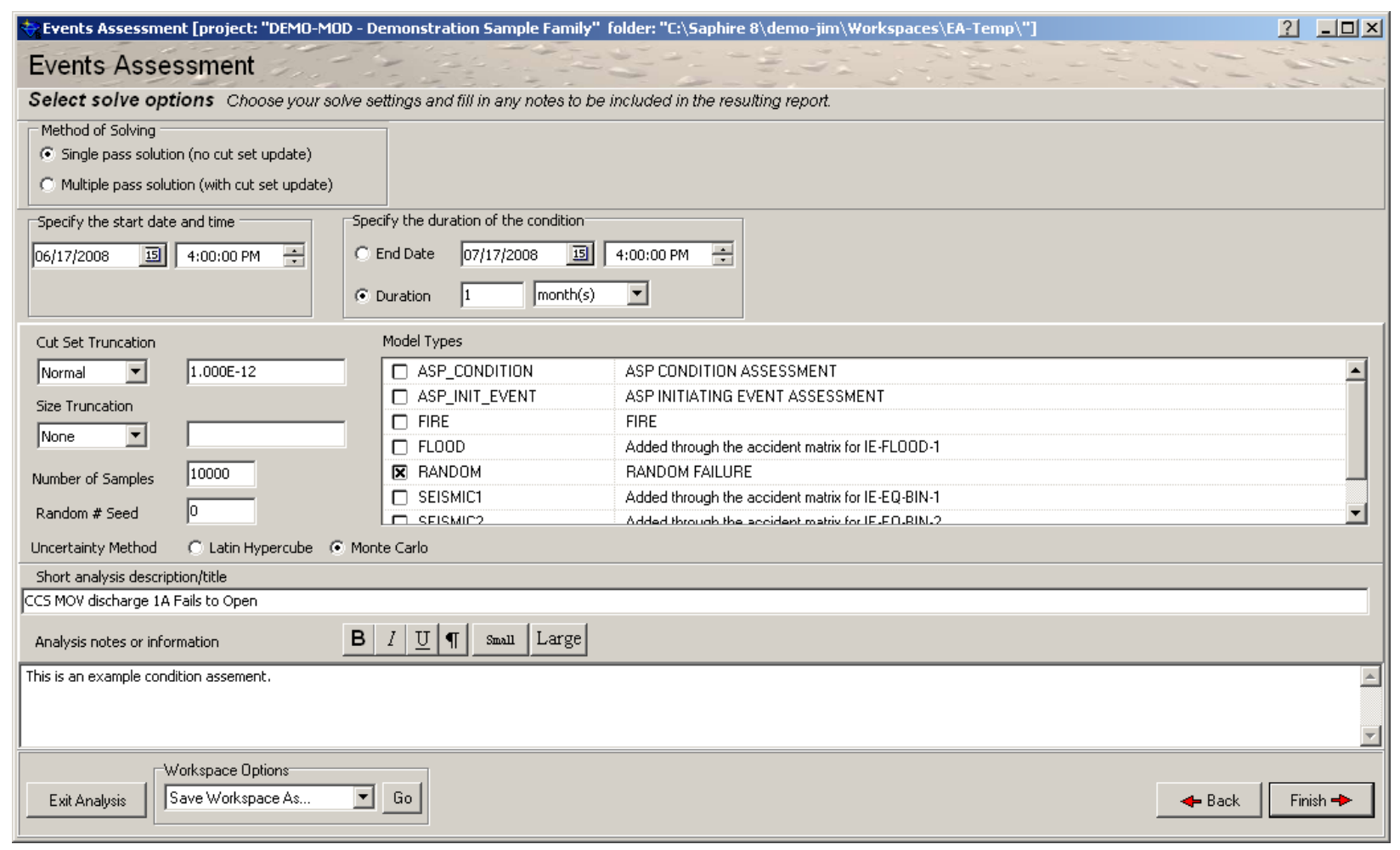

Figure 62. EA Solve options screen for Condition Assessment

Now that all of the information has been specified, click the Finish button to analyze the event. SAPHIRE will provide an output of the assessment on the last screen (Figure 63). 
Events Assessment

06/17/2008 4:12:17 PM
Demonstration Sample Family in C: Saphire 81demojim Workspaces\EA-Temp\}

Condition Assessment

\section{Condition Assessment Summary}

\begin{tabular}{ll} 
Event Date & $06 / 17 / 20084: 00: 00$ PM to $07 / 17 / 20084: 00: 00$ PM \\
\hline Duration & 1 month \\
\hline CCDP & $4.352 \mathrm{E}-6$ \\
\hline CDP & $4.351 \mathrm{E}-6$ \\
\hline$\Delta$ CDP & $1.667 \mathrm{E}-9$
\end{tabular}

CCS MOV discharge 1 A Fails to Open

\section{Summary of Event Changes}

\begin{tabular}{llll} 
Event & Description & New value & Nominal Value \\
\hline CCS-MOV-CC-1A & CCS Train 1A pump discharge isolation valve & True & $5.000 E-3$ \\
\hline
\end{tabular}

\section{CCDP Uncertainty Distribution}

\begin{tabular}{lllll}
$5 \%$ & Median & $\begin{array}{l}\text { Point } \\
\text { Estimate }\end{array}$ & Mean & $95 \%$ \\
\hline $5.868 \mathrm{E}-7$ & $8.342 \mathrm{E}-7$ & $1.093 \mathrm{E}-6$ & $1.149 \mathrm{E}-6$ & $2.561 \mathrm{E}-6$
\end{tabular}

\section{Event Tree Dominant Results}

Only items contributing at least $1.0 \%$ to the total CCDP are displayed.

\begin{tabular}{lllll}
\hline \hline EVENT TREE & CCDP & CDP & $\Delta$ CDP & DESCRIPTION \\
\hline LOSP & $4.352 \mathrm{E}-6$ & $4.351 \mathrm{E}-6$ & $1.667 \mathrm{E}-9$ & Loss of Offsite Power Event Tree \\
\hline Total & $4.352 \mathrm{E}-6$ & $4.351 \mathrm{E}-6$ & $\mathbf{1 . 6 6 7 \mathrm { E } - 9}$ & \\
\hline
\end{tabular}

\section{Dominant Sequence Results}

Only items contributing at least $1.0 \%$ to the total CCDP are displayed.

\begin{tabular}{|c|c|c|c|c|c|}
\hline EVENT TREE & SE OUENCE & $\underline{\text { CCDP }}$ & $\triangle \mathrm{CDP}$ & DESCRIPTION & \\
\hline \multicolumn{3}{|c|}{ Workspace 0ptions } & Report Options & & \\
\hline Exit Analysis & e Workspace As... & \pm Go & Print Report... & $\Rightarrow$ Go & \& Back \\
\hline
\end{tabular}

Figure 63. EA Results screen for Condition Assessment 
The calculation part that SAPHIRE does to obtain the $\triangle \mathrm{CDP}$ shown in the Analysis Results page is

$$
\begin{aligned}
& \Delta \mathrm{CDP}=\mathrm{CCDP}-\mathrm{CDP} \\
& \text { where: } \\
& \mathrm{CCDP}=\text { conditional core damage probability of event }=\mathrm{CCDF} * \text { duration } \\
& \mathrm{CDP}=\text { core damage probability }=\text { nominal } \mathrm{CDF} * \text { duration }
\end{aligned}
$$

The uncertainty results that is shown above is based on sampling from the component's variability and then solving for the CCDF and CDF based on the sampled probability. SAPHIRE then subtracts CDF from CCDF to obtain a sampled $\triangle \mathrm{CCDF}$, which is multiplied by the duration. SAPHIRE stores this calculated value and continues this process for the number of samples noted. Once SAPHIRE has performed the number of specified samples, it orders the $\triangle$ CCDP values and pulls out the $5^{\text {th }}, 50^{\text {th }}$, and $95^{\text {th }}$, then calculates the mean by summing up the $\triangle$ CCDPs and divides it by the number of samples.

\section{SOLVING PROJECT - General Analysis}

This set of analysis is completely new in SAPHIRE 8. The General Analysis Workspace allows for a user defined analysis option. This option provides the user the flexibility to set up an analysis that is saved for future analyses and modifications. To start this process, the user selects General Analysis $\rightarrow$ New Analysis from the Workspaces menu option.

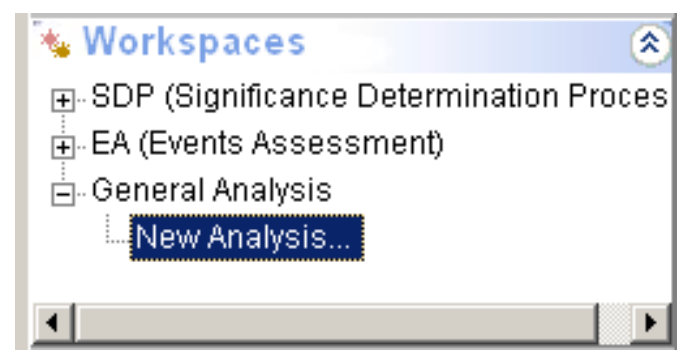

Figure 64. General Analysis workspace path

This option requires a double click and then SAPHIRE copies all of the information from the project into a new workspace. By making this copy, any event tree/fault tree modifications will only be saved for this particular analysis and the original models will not be affected. SAPHIRE 8 then loads up a new analysis screen which allows the user to select what parts of the model will be affected by the analysis by checking the applicable boxes (Figure 65). 


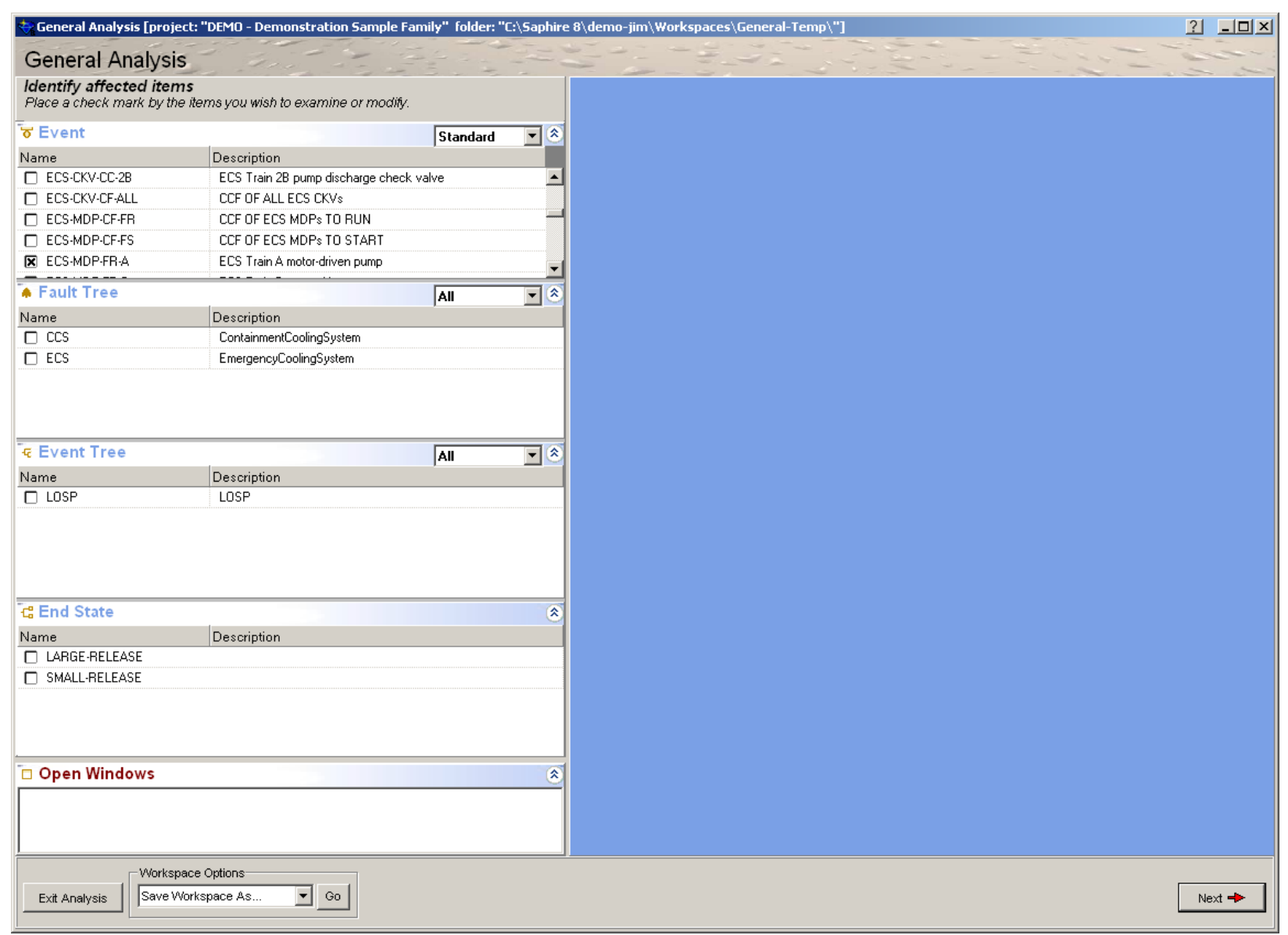

Figure 65. General Analysis start screen

The basic events, fault trees and event trees are checked in order to tell SAPHIRE 8 that these parts of the project are going to be affected for this particular analysis. Once the parts of the model are checked, then the Next button is selected.

This screen (Figure 66) is used to modify those parts of the project that were selected. The basic event probabilities can be changed to a new probability, probability of 1.0, True house event, or False house event. The difference between a probability of 1 and True deals primarily with how the common cause failure (CCF) events are recalculated for the analysis (Figure 67). 


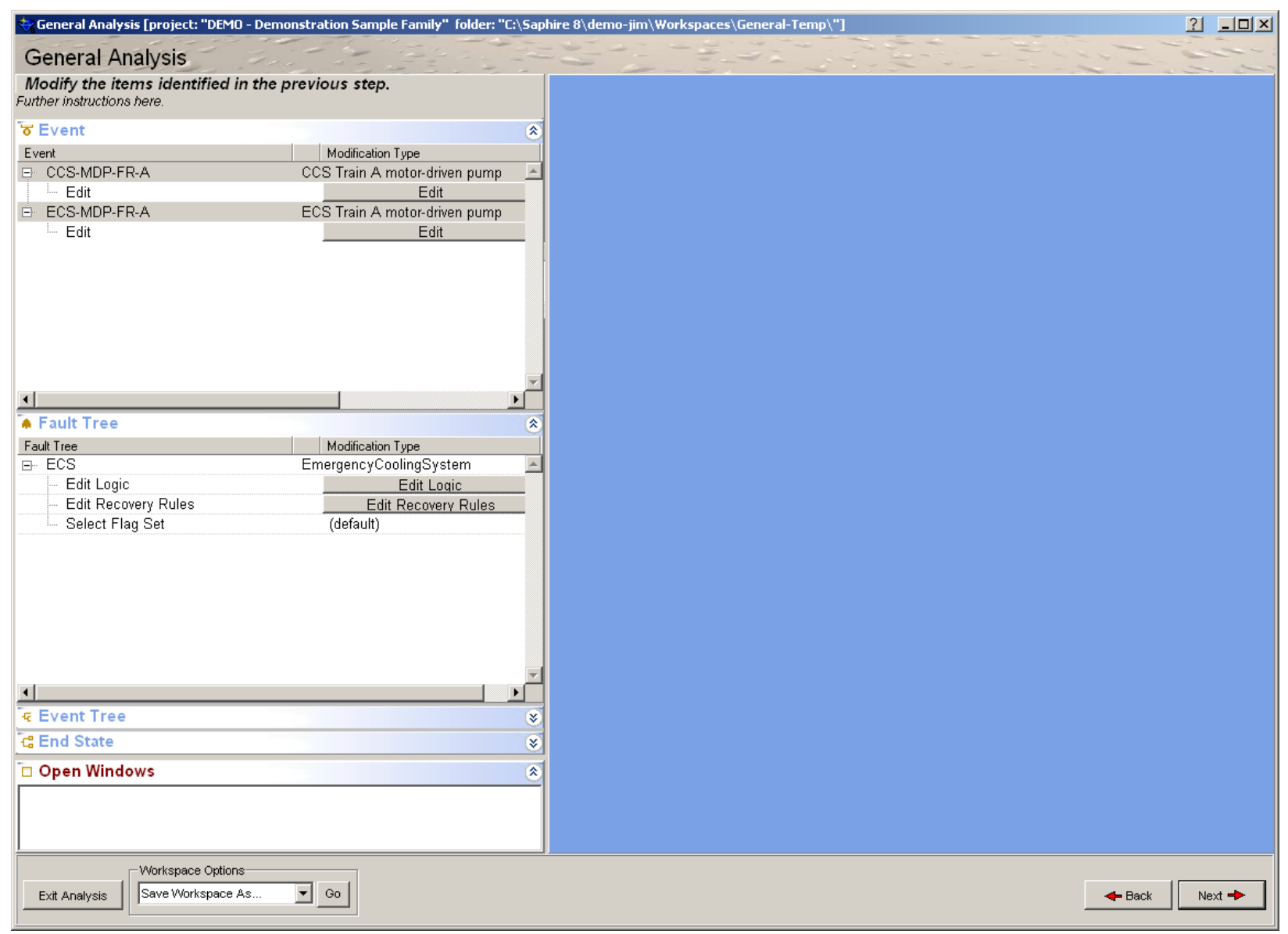

Figure 66. General Analysis modification screen

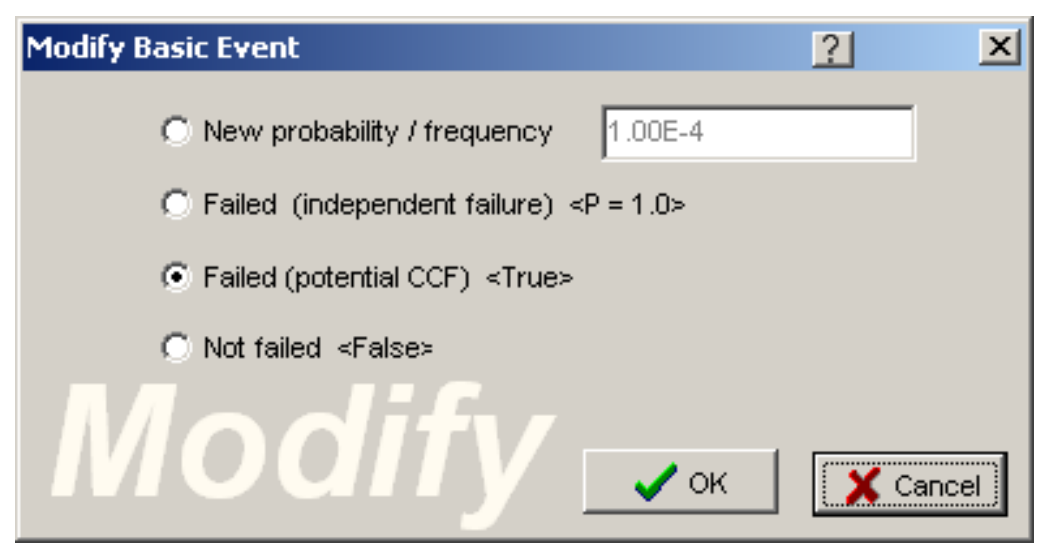

Figure 67. General Analysis Modify Basic Event pop up form

The checked fault tree(s) can now be edited (Figure 68). The fault tree logic can be modified or the recovery rules for this specific analysis. Again, the advantage of this analysis process is the logic will only be affected for this specific workspace - the logic in the original model is left untouched. 


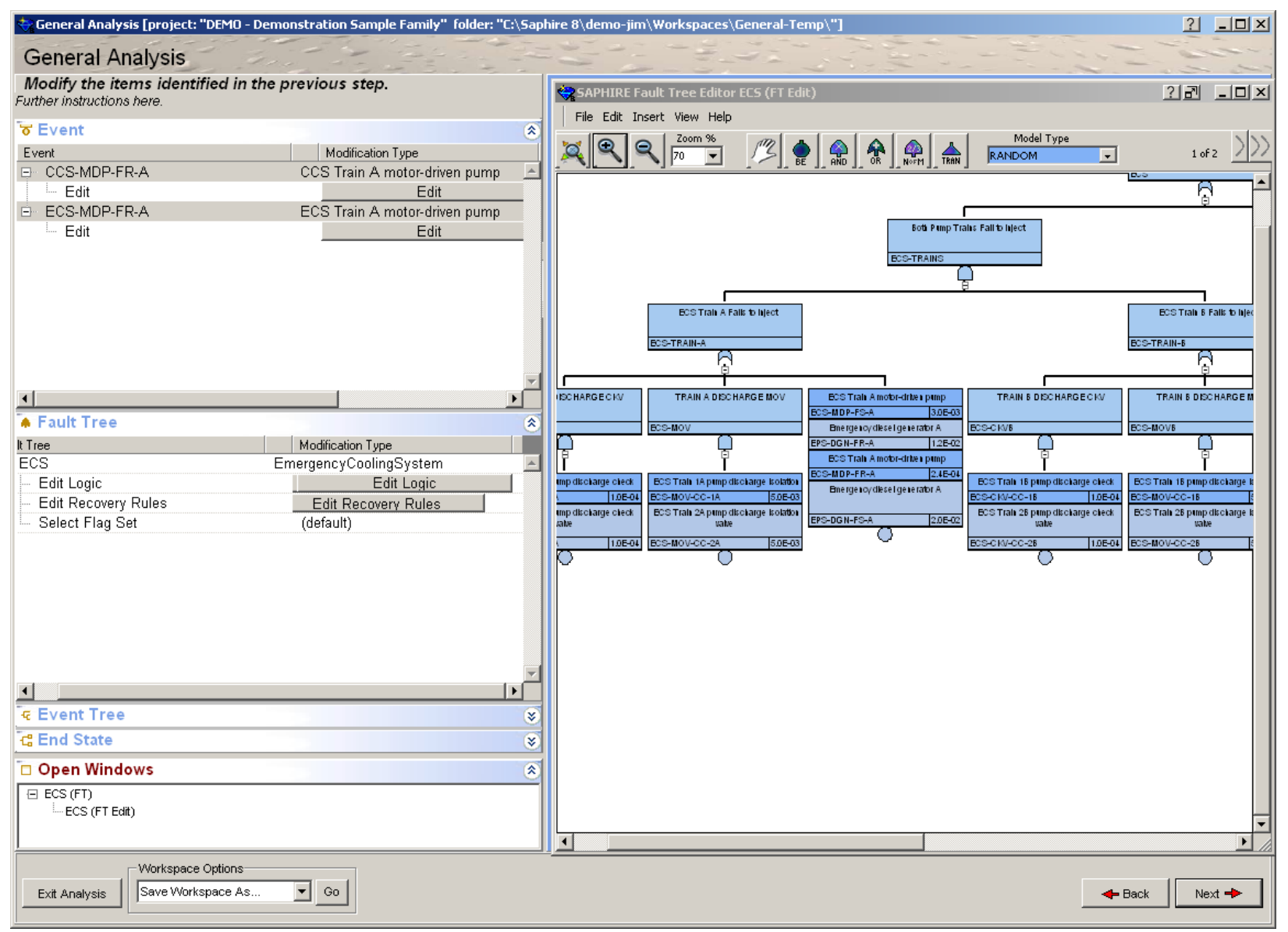

Figure 68. General Analysis Modify screen with Fault Tree editor

The last option is the Select Solve Options (Figure 69). This option allows the user the flexibility of what event trees, fault trees, end states, and model types to be analyzed. The individual event trees or fault trees can be selected or all event trees and fault trees can be selected. Once the selections are made, click the Finish button.

SAPHIRE 8 has two options when solving the model:

- Single pass with no cut set update solution

- Multiple pass with cut set update solution

SAPHIRE 8 follows the steps below for the "single-pass" solution:

1. Recalculate the "nominal case" to be able to determine the increase in risk.

a. Generate basic event data with no change sets marked. If the no test/maintenance option is used, all T\&M events will be set to zero.

b. Solve all sequence cut sets using a predetermined (but low) truncation.

c. Apply recovery rules to all sequence cut sets.

d. If identified in the project constants, perform a cut set update to ensure nonminimal cut sets are removed. 
e. Re-quantify the cut sets.

f. Store the cut set results via a base case update.

2. Calculate the risk increase.

a. Generate basic event data with SDP changes indicated by the analyst. If the no test/maintenance option is used, all T\&M events will be set to zero.

b. Solve sequence cut sets using the same truncation for the "nominal" case.

c. Apply recovery rules to all sequence cut sets.

d. If identified in the project constants, perform a cut set update to ensure nonminimal cut sets are removed.

e. Re-quantify the cut sets.

f. Store the cut set results for use in determining the SDP annualized CDF.

SAPHIRE 8 follows the steps below for the "multi-pass" solution:

1. Recalculate the "nominal case" to be able to determine the increase in risk.

a. Generate basic event data with no change sets marked. If the no test/maintenance option is used, all T\&M events will be set to zero.

b. Solve all sequence cut sets using a predetermined (but low) truncation.

c. Apply recovery rules to all sequence cut sets.

d. Perform a cut set update to ensure non-minimal cut sets are removed.

e. Re-quantify the cut sets to ensure they have the proper values.

f. Store the cut set results via a base case update.

2. Calculate the risk increase.

a. Generate basic event data with SDP changes indicated by the analyst and store these values for use in Step $2 \mathrm{~g}$. If the no test/maintenance option is used, all T\&M events will be set to zero.

b. Change TRUE events to 1.0 events (to ensure application of recovery rules).

c. Solve sequence cut sets using the same truncation for the "nominal" case.

d. Apply recovery rules to all sequence cut sets.

e. Change events having a probability of 1.0 to TRUE events (to ensure that nonminimal cut sets will be removed during the next step).

f. Perform a cut set update to ensure non-minimal cut sets are removed.

g. Re-quantify the cut sets so they have the proper values stored from step 2a.

h. Store the cut set results for use in determining the SDP annualized CDF.

The following example will provide the results of the single and multi-pass processes and the use of 1.0 versus True for the Train A CCS and ECS motor-driven pumps to run in the DEMO project. 


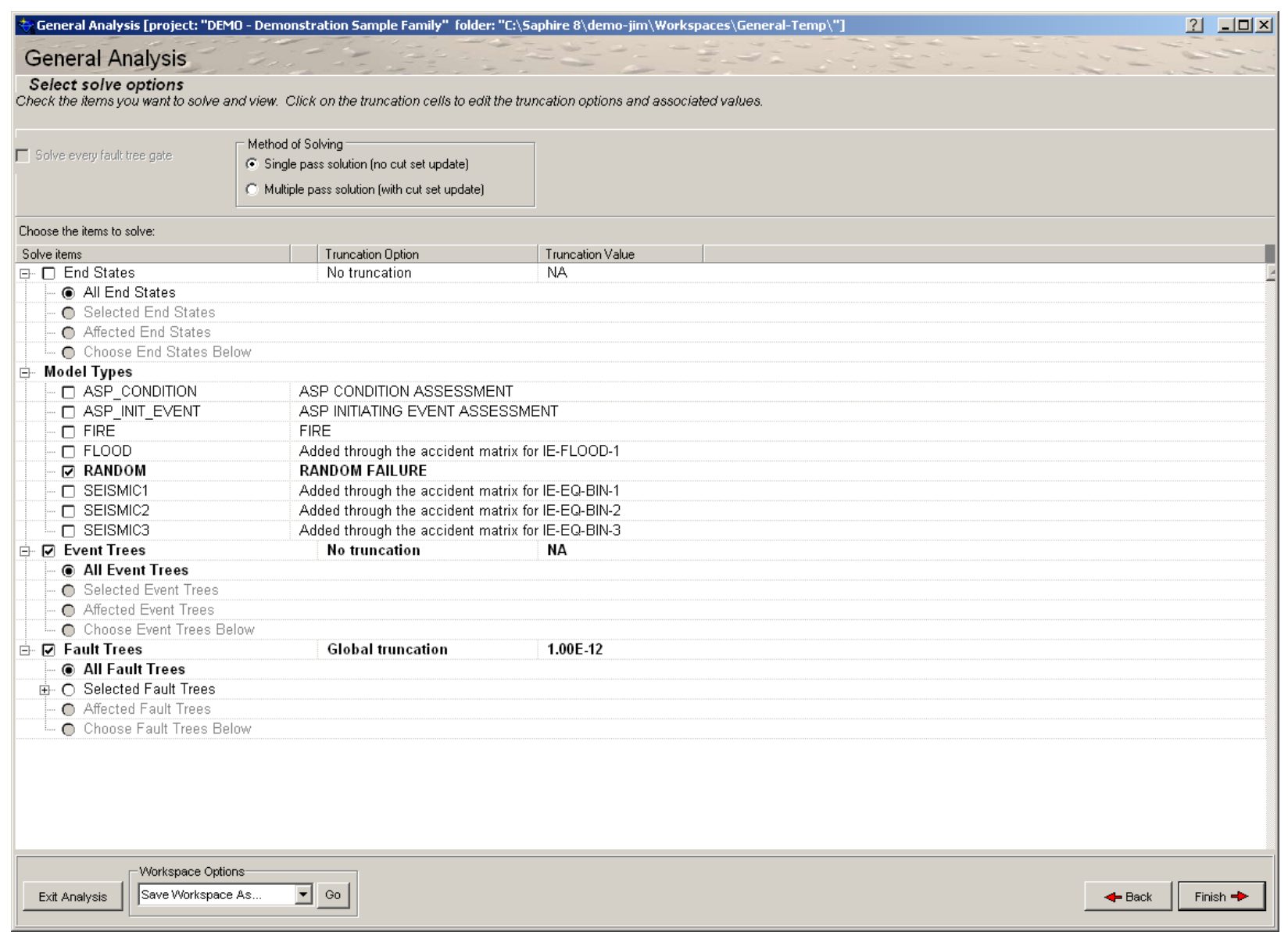

Figure 69. General Analysis Solve options screen

SAPHIRE will show a prompt expressing that the following analysis could take some time to complete (Figure 48) and allows the analyst to decide to perform the analysis (which is a complete re-solve of all logic models). Clicking Cancel will just show existing results (if available).

Once the analysis is complete, a View Results screen is displayed (Figure 70). This screen is used to provide the final output result for the event trees, fault trees, and end states. 


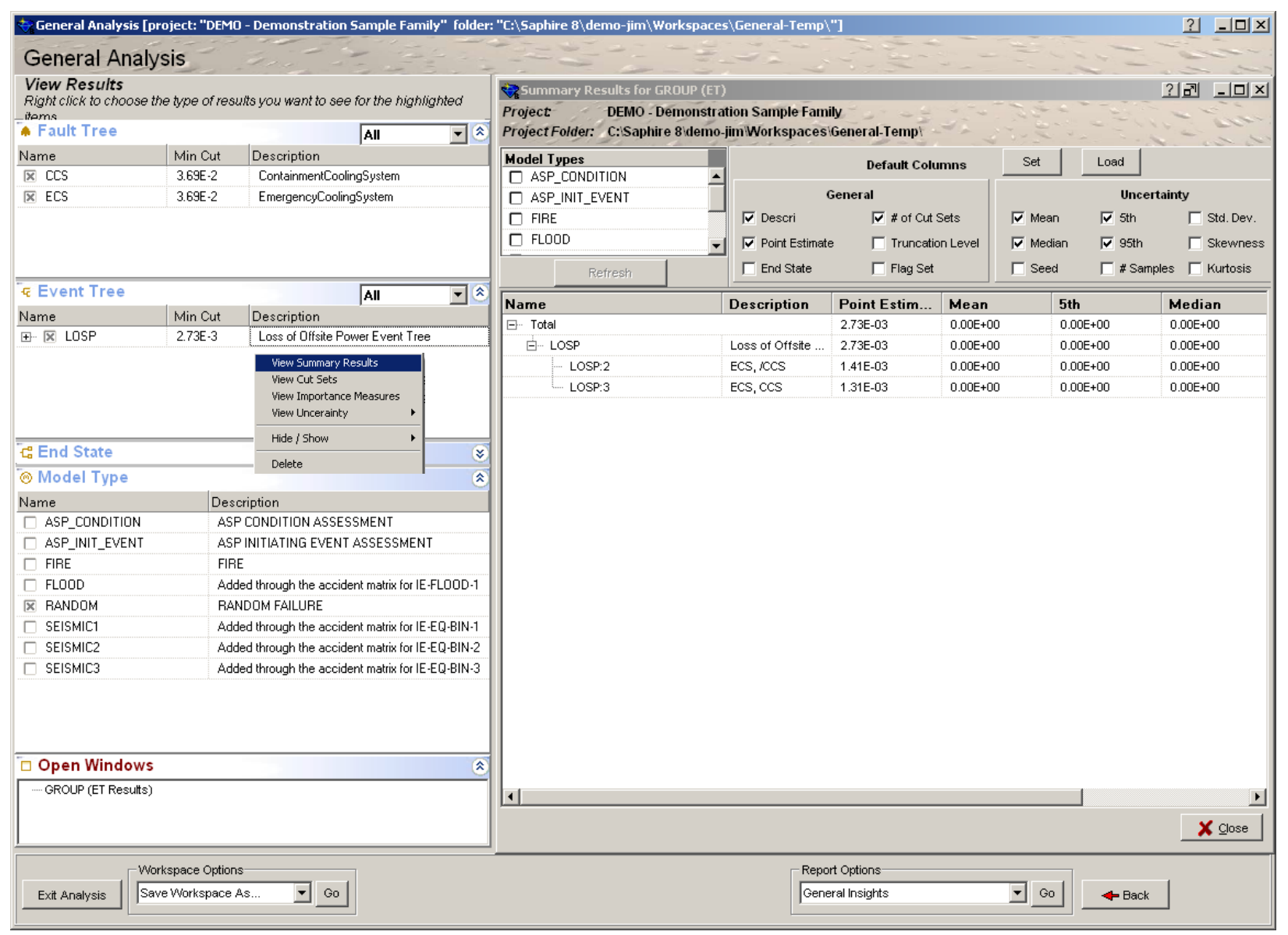

Figure 70. General Analysis results screen

The overall results for the fault trees and the event tree analysis are shown in the left column. With the LOOP event tree highlighted and clicking the View Summary Results, the overall summary is shown in the right field. The results are based on only analyzing the fault trees and event tree accident sequences with the component's probabilities set to 1.0. SAPHIRE will generate the minimal cut sets based on the input information and then apply the recovery rules, since the single pass analysis was selected. The resultant cut sets are shown below (Figure 71). 


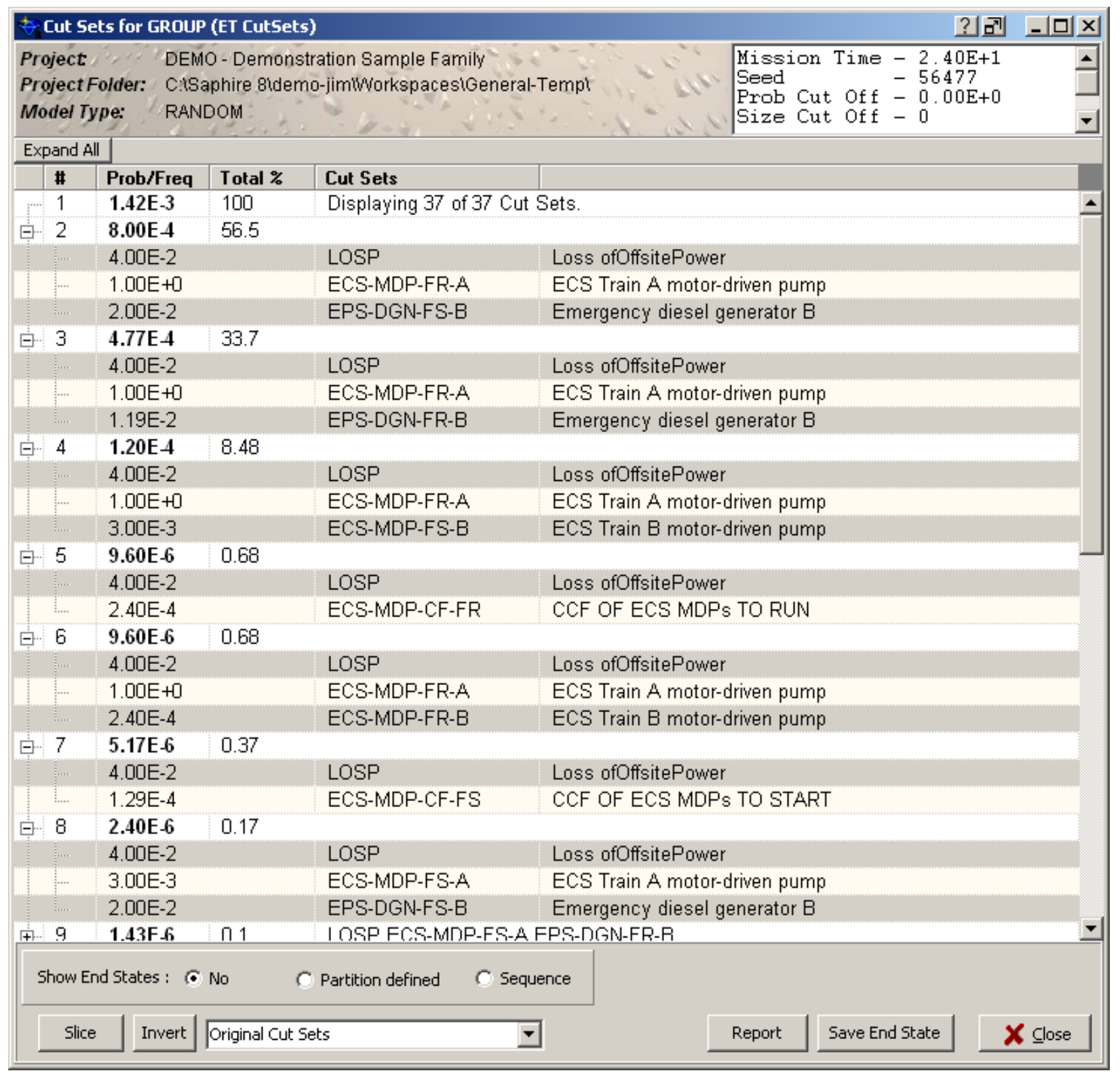

Figure 71. General Analysis Cut Set results

As can be seen in the figure above, since the basic event (ECS-MDP-FR-A) was set to 1.0, SAPHIRE solves the logic model first and then quantifies the resultant cut sets. SAPHIRE assumes that the basic event can fail (numerically it is) and therefore, it shows up in the cut sets.

Now if the same analysis is performed except to tell SAPHIRE to use multiple passes. SAPHIRE will first solve the logic model and get the same cut sets above, but then it will go back through and remove the failed basic event and then remove the non-minimal cut sets. The results of this analysis are shown below (Figure 72 and Figure 73). 


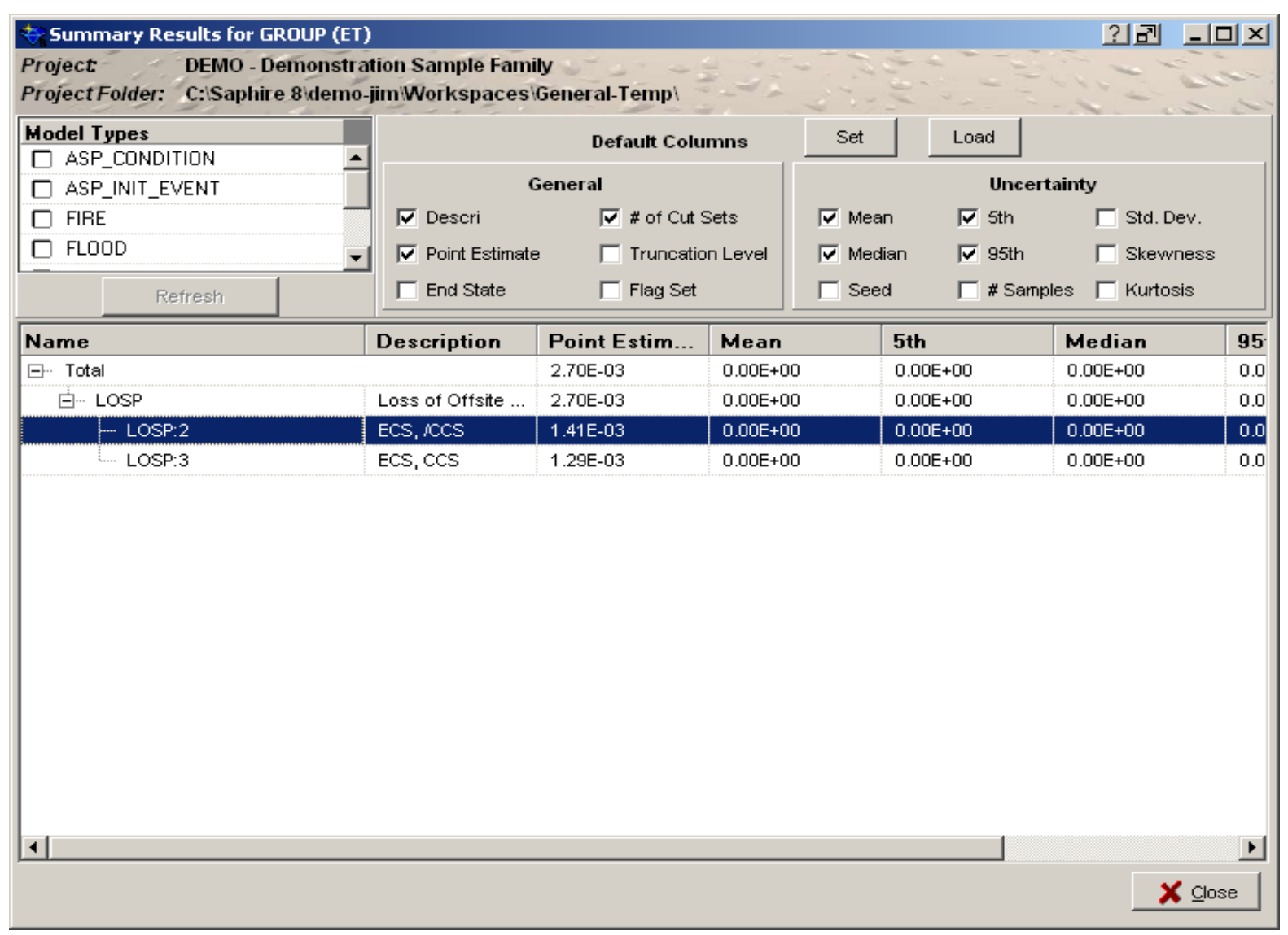

Figure 72. General Analysis multiple pass summary results 


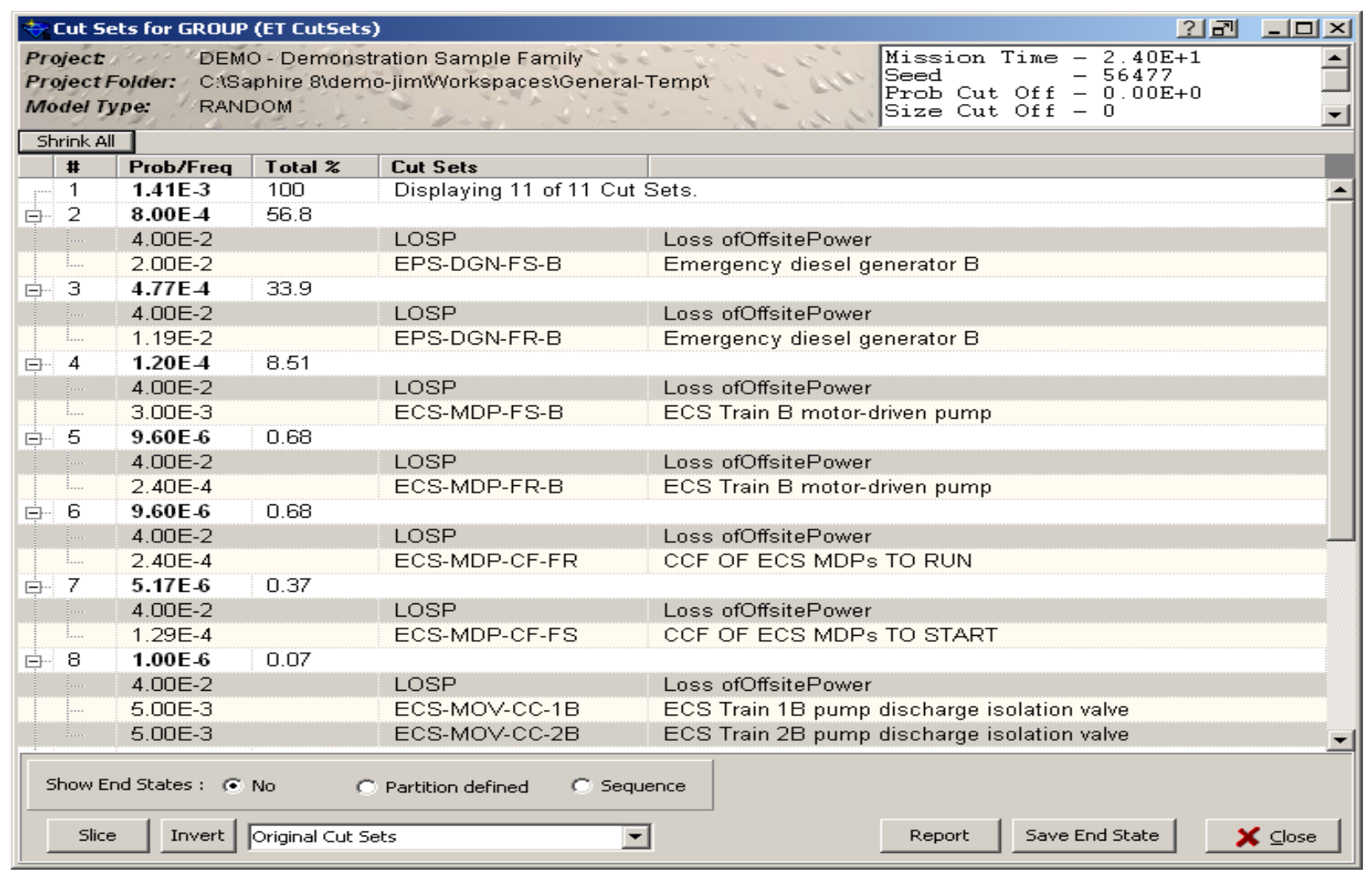

Figure 73. General Analysis multiple pass Cut Set results

As can be seen the total number of cut sets for accident sequence 2 went from 37 cut sets to 11 . Therefore, by performing the second pass (cut set update) 26 non-minimal cut sets were removed from the final results. The results shown above with the multiple pass option selected is an approximate answer given the assumption that the component failed and its failure has no impact on the common cause failure.

The last option for this analysis is to assume that the failure of the same components does affect the common cause failure event. This analysis will set the component to True and multiple pass option will be performed. The results are shown in Figure 74. 


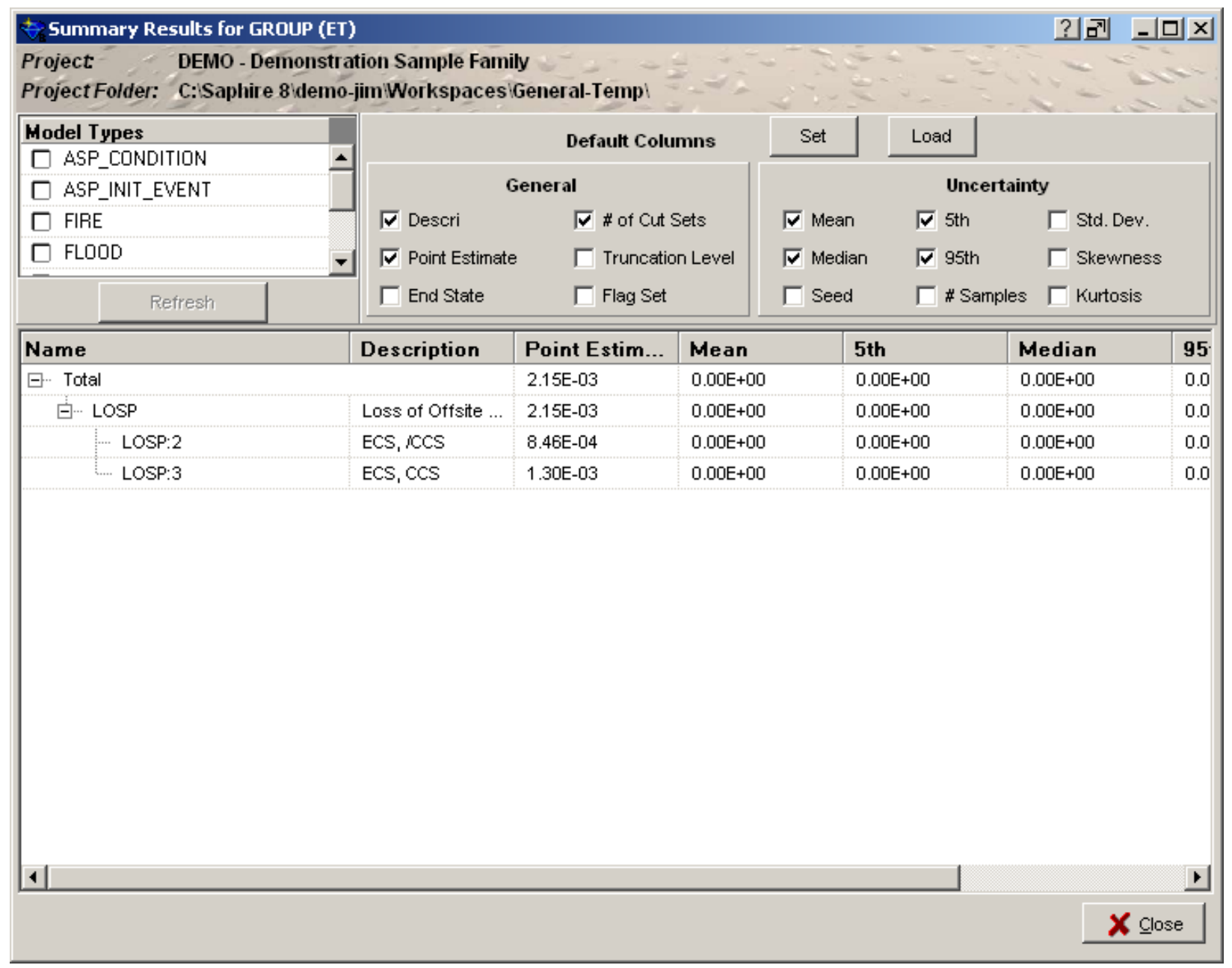

Figure 74. General Analysis summary results with component to True and multiple pass performed

The results from this analysis show that accident sequence 3 is approximately the same, with the exception of the adjustment to the common cause failure basic events. However, accident sequence 2 is completely different. The reason for this is the component is guaranteed to fail and the logic model that is used to generate cut sets is different than when the component is set to 1.0. Therefore, the delete term process works correctly for the option when the component is set to True. The resultant cut sets are shown below (Figure 75). 


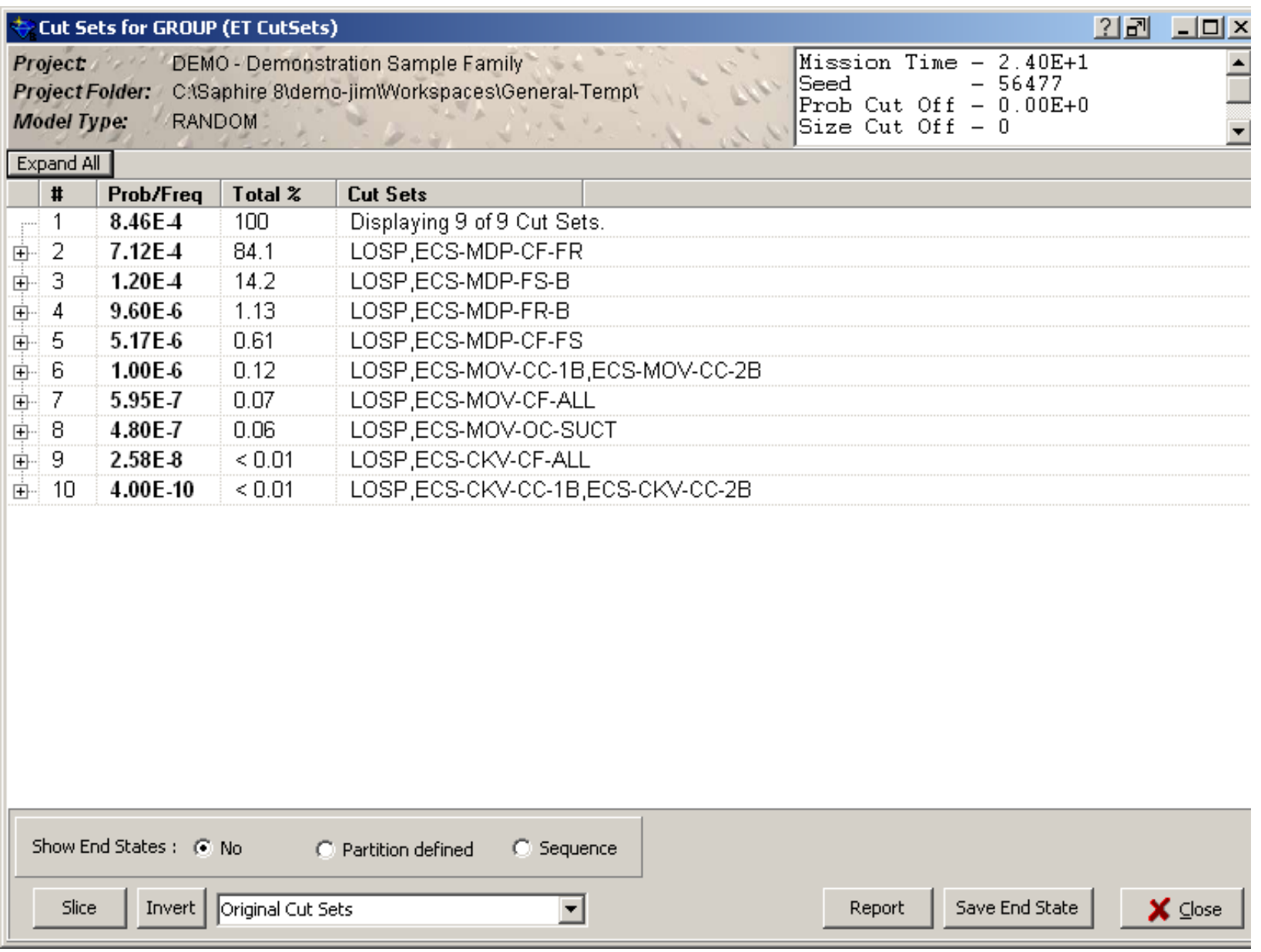

Figure 75. General Analysis Cut Set results with component to True and multiple pass performed 